\title{
Improved Combinatorial Algorithms for the Inhomogeneous Short Integer Solution Problem
}

\author{
Shi Bai \\ Department of Mathematical Sciences, Florida Atlantic University, Boca Raton, USA
}

Steven D. Galbraith (D)

Department of Mathematics, University of Auckland, Auckland, New Zealand

s.galbraith@math.auckland.ac.nz

Liangze Li

School of Mathematical Sciences, Peking University, Beijing, China

Daniel Sheffield

Department of Mathematics, University of Auckland, Auckland, New Zealand

Communicated by Phong Nguyen.

Received 27 November 2014 / Revised 14 September 2018

Online publication 15 October 2018

\begin{abstract}
The paper is about algorithms for the inhomogeneous short integer solution problem: given $(\mathbf{A}, \mathbf{s})$ to find a short vector $\mathbf{x}$ such that $\mathbf{A x} \equiv \mathbf{s}(\bmod q)$. We consider algorithms for this problem due to Camion and Patarin; Wagner; Schroeppel and Shamir; Minder and Sinclair; Howgrave-Graham and Joux (HGJ); Becker, Coron and Joux (BCJ). Our main results include: applying the Hermite normal form (HNF) to get faster algorithms; a heuristic analysis of the HGJ and BCJ algorithms in the case of density greater than one; an improved cryptanalysis of the SWIFFT hash function; a new method that exploits symmetries to speed up algorithms for Ring-SIS in some cases.
\end{abstract}

Keywords. Short integer solution problem (SIS), SWIFFT hash function, Subset-sum, Knapsacks.

\section{Introduction}

The subset-sum problem (also called the knapsack problem) is: Given positive integers $a_{1}, \ldots, a_{m}$ and an integer $s$, to compute a vector $\mathbf{x}=\left(x_{1}, \ldots, x_{m}\right) \in\{0,1\}^{m}$ if it exists, such that

$$
s=\sum_{i=1}^{m} a_{i} x_{i} .
$$


It is often convenient to write $\mathbf{a}=\left(a_{1}, \ldots, a_{m}\right)$ as a row and $\mathbf{x}=\left(x_{1}, \ldots, x_{m}\right)^{T}$ as a column so that $s=\mathbf{a x}$. The modular subset-sum problem is similar: Given a modulus $q$, integer vector $\mathbf{a}$ and integer $s$ to find $\mathbf{x} \in\{0,1\}^{m}$, if it exists, such that $s \equiv \mathbf{a x}(\bmod q)$.

The vector version of this problem is called the inhomogeneous short integer solution problem (ISIS): Given a modulus $q$, a small set $\mathcal{B} \subseteq \mathbb{Z}$ that contains 0 (e.g., $\mathcal{B}=\{0,1\}$ or $\{-1,0,1\}$ ), an $n \times m$ matrix $\mathbf{A}$ (where typically $m$ is much bigger than $n$ ), and a column vector $\mathbf{s} \in \mathbb{Z}_{q}^{n}$ to find a column vector $\mathbf{x} \in \mathcal{B}^{m}$ (if it exists) such that

$$
\mathbf{s} \equiv \mathbf{A x} \quad(\bmod q)
$$

If we want to be more precise we call this the $(m, n, q, \mathcal{B})$-ISIS problem. The original short integer solution problem (SIS) is the case $\mathbf{s}=0$, in which case it is required to find a solution $\mathbf{x} \in \mathcal{B}^{m}$ such that $\mathbf{x} \neq \mathbf{0}$. Our algorithms solve both problems.

We unify the subset-sum and ISIS problems as the $(G, m, \mathcal{B})$-ISIS problem where $G$ is an abelian group (written additively and viewed as a $\mathbb{Z}$-module), $m$ an integer and $\mathcal{B}$ a small subset of $\mathbb{Z}$ that contains 0 . Given $m$ group elements and a target group element from $G$, the $(G, m, \mathcal{B})$-ISIS problem is to write the target element as an integral linear combination with coefficients in $\mathcal{B}$ of the given $m$ group elements. Micciancio [22] gave a similar generalization that he called "generalization of the compact knapsack problem to arbitrary rings," but his paper mostly considers the special case of a ring with a "convolution" product (similar to the rings used in NTRU). Gama, Izabachène, Nguyen and Xie [13] provide a similar abstraction but focus on the worst-to-average reduction of the so-called generalized SIS/LWE problem. Our work mainly focuses on the cryptanalysis aspects. The three motivating examples for the group are $G=\mathbb{Z}$, $G=\mathbb{Z}_{q}$ and $G=\mathbb{Z}_{q}^{n}$. In the latter two cases we consider the following distribution on ISIS instances parameterized by $m \in \mathbb{N}$ : Sample uniformly $\mathbf{A} \in G^{m}$ and $\mathbf{s} \in G$, and a solution is any vector $\mathbf{x} \in \mathcal{B}^{m}$ (if one exists) such that $\mathbf{s}=\mathbf{A x}$. In the case $G=\mathbb{Z}$ one would need to specify a distribution on elements of $\mathbb{Z}^{m}$ in order to define a distribution on ISIS instances. We do not explore the case $G=\mathbb{Z}$ in this paper, as our algorithms are suited to the modular case.

We now define the density of an ISIS instance, generalizing a standard concept in the subset-sum problem over $\mathbb{Z}$ with $\mathcal{B}=\{0,1\}$. If one chooses the integers $a_{i}$ in a subsetsum instance uniformly in an interval $\{0,1, \ldots, B\} \subset \mathbb{Z}$ then $\max \left\{a_{i}\right\} \approx B$ and the standard definition of density is $m / \log _{2}\left(\max \left\{a_{i}\right\}\right) \approx m / \log (B)=\log \left(\# \mathcal{B}^{m}\right) / \log (B)$. Taking $G=\mathbb{Z}_{B}$, our definition below gives density $m / \log (B)$. For our purposes it is also convenient to define an alternative notion of density that we call exponential density.

Definition 1. Let $G$ be a finite group. The density of a $(G, m, \mathcal{B})$-ISIS instance is $\frac{m \log (\# \mathcal{B})}{\log (\# G)}$. The exponential density of a $(G, m, \mathcal{B})$-ISIS instance is $\delta=\frac{(\# \mathcal{B})^{m}}{\# G}$.

To interpret exponential density, we recall the notion of regularity. Lemma 1 of Ajtai [2] proves regularity of this construction for any finite abelian group $G$ and $\mathcal{B}=\{0,1\}$, meaning that for any $\epsilon>0$ there is an integer $m$ so that if $\mathbf{A}$ is a randomly chosen vector in $G^{m}$ then the statistical distance of the uniform distribution on $G$ from the distribution $\left\{\mathbf{A x}: \mathbf{x} \in\{0,1\}^{m}\right\}$ is at most $\epsilon$. In light of this result, it is natural to model the values $\mathbf{A x}$ over $\mathbf{x} \in\{0,1\}^{m}$ as if they are sampled independently and uniformly from $G$. We state 
this heuristic assumption precisely as Heuristic 1 below. With Heuristic 1 , when $\delta<1$ we consider the exponential density to be a close approximation to the probability, given uniform $\mathbf{s}$, that there is a solution $\mathbf{x} \in \mathcal{B}^{m}$ for the equation $\mathbf{s}=\mathbf{A x}$. Similarly, when $\delta>1$ we consider $\delta$ to be a close approximation to the average size of the solution set $\left\{\mathbf{x} \in \mathcal{B}^{m}: \mathbf{s}=\mathbf{A} \mathbf{x}\right\}$.

Throughout the paper the phrases "low density" and "high density" are used informally, and are consistent with either definition of density. If $\# \mathcal{B}^{m}$ is much smaller than $\# G$ then we say the $(G, m, \mathcal{B})$-ISIS problem has low density. If $\# \mathcal{B}^{m} \approx \# G$, then we say we are in the density 1 case. If $\# \mathcal{B}^{m}$ is much larger than $\# G$, then we are in the high-density case.

The ISIS problem has applications in lattice-based cryptography. For example, inverting the SWIFFT hash function of Lyubashevsky, Micciancio, Peikert and Rosen [21] is solving $(1024,64,257,\{0,1\})$-ISIS. Since this function is a compression function (mapping 1024 bits to about 512 bits) it corresponds to a very high-density instance of ISIS. The security level of SWIFFT claimed in [21] is "to find collisions takes time at least $2^{106}$ and requires almost as much space, and the known inversion attacks require about $2^{128}$ time and space". ${ }^{1}$ Note that the main contribution of the SWIFFT paper [21] was to propose and implement the new hash function, and hence only provided a very informal security estimate. Our estimates are that the algorithms sketched in [21] actually require around $2^{148}$ and $2^{153}$ bit operations, respectively. Appendix B of [16] (an early version of [15]) gives an improved collision attack, exploiting the birthday paradox, using lists of size $2^{96}$ (surprisingly this result is missing in the published version [15]). Our estimates are that this algorithm requires around $2^{115}$ bit operations. The unpublished preprint of Kirchner [19] claims a collision attack on SWIFFT that requires $2^{109}$ bit operations, but the method is sketchy and no detailed analysis is given. We remark that a stronger variant of the SWIFFT hash function has also been proposed [1], but we do not discuss it further in this paper.

It is known that one can try to solve both subset-sum and ISIS using lattice methods (for example, reducing to the closest vector problem (CVP) or shortest vector problem (SVP) in certain lattices of dimension $m$ or $m+1$ ). For instance, given an ISIS problem $\mathbf{s} \equiv \mathbf{A x}$ $(\bmod q)$, one can reduce it to CVP: we consider the lattice $\Lambda_{q}^{\perp}(\mathbf{A})=\left\{\mathbf{y} \in \mathbb{Z}^{m}: \mathbf{A y} \equiv 0\right.$ $(\bmod q)\}$ and find any vector $\mathbf{w} \in \mathbb{Z}^{m}$ such that $\mathbf{A w} \equiv \mathbf{s}(\bmod q)$, then solve the CVP problem (for lattice $\Lambda_{q}^{\perp}(\mathbf{A})$ and target point $\mathbf{w}$ ) to find some $\mathbf{y}$ close to $\mathbf{w}$ and so returns $\mathbf{w}-\mathbf{y}$ as the ISIS solution.

The focus in this paper is on combinatorial algorithms that are based on time-memory tradeoffs. In particular, all approaches described in this paper use exponential storage. In another direction, recent work of Bansal, Garg, Nederlof and Vyas [3] gives a rigorous space-efficient algorithm for the integer subset-sum problem that runs in $\tilde{O}\left(2^{0.86 m}\right)$. By comparison, our heuristic algorithms aim to minimize the time-complexity and are exponentially faster (but also take more space). The main application is to help select parameters for lattice-based cryptosystems: It is important to take into account both lattice algorithms and combinatorial algorithms to determine the concrete security level.

\footnotetext{
${ }^{1}$ We remark that generic hash function collision algorithms such as parallel collision search would require at least $2^{256}$ bit operations. Hence we do not consider such algorithms further in this paper.
} 
In this work, we assume that the set $\mathcal{B}$ is rather small (e.g., $\mathcal{B}=\{0,1\}$ or $\{-1,0,1\}$ ). Some previous algorithms of this type for the subset-sum and ISIS problems are due to: Schroeppel and Shamir; Camion and Patarin; Wagner; Minder and Sinclair; HowgraveGraham and Joux; Becker, Coron and Joux. We will recall the previous algorithms in Sect. 2. It can be seen that the Camion-Patarin/Wagner (CPW) and Minder-Sinclair methods are suitable for very high-density instances (such as SWIFFT), while the other methods are more suitable for low-density instances.

\subsection{Our Contributions}

Our contribution is to give a general framework that unifies the subset-sum, modular subset-sum and ISIS problems. We show that the algorithms by Schroeppel and Shamir, Camion and Patarin, Wagner, Minder-Sinclair, Howgrave-Graham and Joux, Becker-Coron-Joux can be used to solve these generalized problems. The four technical contributions of our paper are:

1. To develop variants of these algorithms for the approximate-ISIS problem (namely $\mathbf{s} \equiv \mathbf{A x}+\mathbf{e}(\bmod q)$ for a "small" $\mathbf{e})$, which itself arises naturally when one takes the Hermite normal form of an ISIS instance.

2. To study the Howgrave-Graham and Joux (HGJ) and Becker-Coron-Joux (BCJ) methods in the case of instances of density greater than one. We give in Figure 1 of Sect. 3 a comparison of the HGJ, BCJ and CPW algorithms as the density grows.

3. To give improved cryptanalysis ${ }^{2}$ of the SWIFFT hash function [21] by reducing it to approximate-ISIS. We reduce the collision attack time from around $2^{113}$ to around $2^{104}$ bit operations (this corresponds to a speedup of factor $\approx 500$ ). We also reduce inverting time by a factor of $\approx 1000$. We stress that our algorithms do not break the SWIFFT hash function, as our running times are not significantly faster than the informal security estimate (lower bound) of $2^{106}$ stated in [21].

4. The SWIFFT hash function and many other cryptographic problems (such as NTRU) are actually based on the Ring-SIS or Ring-ISIS problems (see Sect. 6). The previous analysis of these algorithms has ignored the ring structure. In Sect. 6 we sketch how to speed up algorithms for Ring-SIS by exploiting symmetries. Our main insight is to choose a suitable eigenbasis that allows to include symmetries into our general framework for the algorithms. These ideas do not seem to be compatible with the use of the Hermite normal form, and so do not give further improvements to our attacks on the SWIFFT hash function.

\subsection{Related Literature}

There is an extensive literature on the approximate subset-sum problem over $\mathbb{Z}$ (given $s \in \mathbb{Z}$ to find $\mathbf{x}$ such that $s \approx \mathbf{a x}$ ) including polynomial-time algorithms (see Section 35.5 of [9]). These algorithms exploit properties of the usual ordering on $\mathbb{Z}$ and do not seem to

\footnotetext{
${ }^{2}$ We remark that in [7], the authors claimed that finding pseudo-collisions for SWIFFT is comparable to breaking a 68-bit symmetric cipher. Their method is to reduce to the sublattices of dimension 206. However the pseudo-collision is not useful to find real collisions for SWIFFT, since in dimension 206 the real collisions for SWIFFT almost certainly do not exist.
} 
be applicable to ISIS. Indeed, such algorithms cannot be directly applied to the modular subset-sum problem either, though the modular subset-sum problem can be lifted to polynomially many instances of the subset-sum problem over $\mathbb{Z}$ and then the approximate subset-sum algorithms can be applied. Hence, even though the algorithms considered in our paper can be applied to the subset-sum and modular subset-sum problems, our main interest is in the ISIS problem.

\section{Algorithms to Solve Subset-Sum/ISIS}

In this section, we give a general framework for discussing the algorithms of Camion and Patarin, Wagner, Minder and Sinclair, Howgrave-Graham and Joux, Becker, Coron and Joux. Previously they were always discussed in special cases. First we define the problem. Then, in Sect. 2.1 we list the algorithms and their complexities. Section 2.2 presents the general framework and Sect. 2.3 presents our generalized merge algorithm. The remainder of the section is to show that all previous algorithms can be described and unified as special cases of our general framework.

Definition 2. Let $G$ be a finite abelian group, $m$ an integer and $\mathcal{B}$ a small subset of $\mathbb{Z}$ that contains 0 . The $(G, m, \mathcal{B})$-ISIS problem is defined as follows. An instance of the problem is a pair $(\mathbf{A}, \mathbf{s})$ with $\mathbf{A} \in G^{m}$ and $\mathbf{s} \in G$, and a solution is any vector $\mathbf{x} \in \mathcal{B}^{m}$ (if one exists) such that $\mathbf{s}=\mathbf{A x}$.

The weight of a solution $\mathbf{x}$ is defined to be $\operatorname{wt}(\mathbf{x})=\#\left\{i: 1 \leq i \leq m, x_{i} \neq 0\right\}$. Let $\omega \in \mathbb{N}$. The weight- $\omega(G, m, \mathcal{B})$-ISIS problem is: Given $(\mathbf{A}, \mathbf{s})$, to compute a solution $\mathbf{x} \in \mathcal{B}^{m}$ such that $\mathbf{s}=\mathbf{A x}$ in $G$ and $\operatorname{wt}(\mathbf{x})=\omega$.

Our two main examples for the group are $G=\mathbb{Z}_{q}$ and $G=\mathbb{Z}_{q}^{n}$.

\subsection{Brief Survey of Previous Methods}

It is straightforward that one can solve the $(G, m,\{0,1\})$-ISIS problem in $\tilde{O}\left(2^{m / 2}\right)$ time and the same storage using birthday methods.

Schroeppel and Shamir [26] showed how to match this running time but use considerably less space. A better variant of the Schroeppel-Shamir algorithm was given by Howgrave-Graham and Joux [15]. We briefly recall some details in Sect. 2.4.

The important paper of Howgrave-Graham and Joux [15] (HGJ) broke the $\tilde{O}\left(2^{m / 2}\right)$ barrier, giving a heuristic algorithm to solve the density-one subset-sum in time $\tilde{O}\left(2^{0.337 m}\right)$ and storage around $\tilde{O}\left(2^{0.256 m}\right)$. Note that [15] presents algorithms for the traditional subset-sum problem, but Section 6 of [15] mentions that the methods should be applicable to variants of the subset-sum problem including approximate subset-sum, vector versions of subset-sum (i.e., ISIS), and different coefficient sets (e.g., $x_{i} \in\{-1,0,1\}$ ). Our paper thus addresses these predictions from [15]; we give the details in Sect. 2.7. We also answer the question written in [15] that "It would be interesting to re-evaluate the security of SWIFFT with respect to our algorithm." (The answer is that the SWIFFT hash function has very high density, and so the HGJ algorithm is not faster than other methods; see final paragraph of Sect. 3.1.) 
Becker, Coron and Joux [4] (BCJ) gave some improvements to the HGJ method (also restricted to the setting of subset-sum). We sketch the details in Sect. 2.8.

The basic idea of the so-called $k$-tree algorithm was rediscovered several times. Camion and Patarin [8] gave an algorithm for solving high density subset-sum instances, and similar ideas were used by Wagner [28] for solving the " $k$-sum problem". We will refer to these ideas as the CPW algorithm and present it in Sect. 2.5. The work of Minder and Sinclair [25] explained how to use these ideas more effectively (we sketch the details in Sect. 2.6).

Lyubashevsky [20] noted that the CPW algorithm can be applied to solve high density subset-sum problems. Shallue [27] extended Lyubashevsky's work. Lyubashevsky, Micciancio, Peikert and Rosen [21] explain that the CPW algorithm can be applied to solve ISIS in the high density case (inverting the SWIFFT hash function is a very high density case of ISIS).

\subsection{A General Framework}

All the algorithms mentioned above work by reducing to simpler problems (meaning, smaller solution space) of higher density. In our general framework we express this by taking quotients. Indeed, the main conceptual idea of all these algorithms is that highdensity instances are easier to solve using brute-force/meet-in-middle ideas, so we try to reduce the problem to a simpler problem of high density.

The algorithms feature two basic operations (possibly recursively):

1. Compute lists of solutions to some constrained problem obtained by "splitting" the solution space (i.e., having a smaller set of possible $\mathbf{x}$ ) in a quotient group $G / H$. Splitting the solution space lowers the density, but working in the quotient group $G / H$ compensates by raising the density again.

2. Merge two lists of solutions to give a new list of solutions in a larger quotient group $G / H^{\prime}$. (In the final stage of the algorithm we will have $H^{\prime}=\{0\}$ so that $G / H^{\prime} \cong G$.)

The algorithms differ primarily in the way that splitting is done.

We now introduce our notation of quotients, which will allow us to unify the previous work. Let $H$ be a subgroup of $G$ and write $G / H$ for the quotient. Since the map $G \rightarrow$ $G / H$ is a group homomorphism, an instance $\mathbf{s}=\mathbf{A x}$ in $G$ reduces to an instance $\mathbf{s} \equiv \mathbf{A x}(\bmod H)$ in $G / H$. The exponential density increases from $\frac{(\# \mathcal{B})^{m}}{\# G}$ to $\frac{(\# \mathcal{B})^{m}}{\#(G / H)}$, since the number of possible targets $\mathbf{s}(\bmod H)$ is reduced while the number of inputs $\mathbf{x}$ remains the same. Of course, not every solution to the reduced problem will correspond to a solution to the original problem. But for suitable parameters one may recover the original solution in $G$ by "merging" the solutions in $G / H$. This merging step will be detailed in Sect. 2.3.

In practice we will employ this idea in the following ways: for $G=\mathbb{Z}$ then take $H=$ $M \mathbb{Z}$ and $G / H=\mathbb{Z}_{M}$; for $G=\mathbb{Z}_{q}$ and $M \mid q$ then take $H=M \mathbb{Z}_{q}$ and $G / H \cong \mathbb{Z}_{M}$; for $G=\mathbb{Z}_{q}^{n}$ and $1 \leq \ell<n$ then take $H=\left\{\left(0, \ldots, 0, g_{\ell+1}, \ldots, g_{n}\right)^{T}: g_{i} \in \mathbb{Z}_{q}\right\} \cong \mathbb{Z}_{q}^{n-\ell}$ so that $G / H \cong \mathbb{Z}_{q}^{\ell}$.

Remark 1. Our general framework allows to consider the CPW algorithm for subsetsum and modular subset-sum. To have the above decomposition one needs the modulus 
in the modular subset-sum problem to have factors of a suitable size (in other words, to have suitable subgroups). However, it does not necessarily mean that a group with no subgroups is immune to combinatorial algorithms of the other types. Indeed, Wagner and Lyubashevsky described approaches for modular subset-sum using sub-intervals instead of quotients (for further details we refer to Lyubashevsky [20]). We also mention the work of Shallue [27], which gives a rigorous analysis of the CPW algorithm for the modular subset-sum problem. Similarly, our general formulation in terms of taking quotient groups $G / H$ cannot be applied to solve the elliptic curve discrete logarithm problem. If $G=E\left(\mathbb{F}_{q}\right)$ is an elliptic curve group of prime order then there are no suitable subgroups $H$ to apply quotients. There may be other techniques available when suitable subgroups are not available. We leave this problem for future work.

We also remark that high density instances can often be reduced to smaller dimensional instances having density approximately one: Choose a suitable integer $\ell$ (i.e., so that $(\# \mathcal{B})^{m-\ell} \approx \# G$ ) and set $\ell$ entries of $\mathbf{x}$ to be zero. Delete the corresponding elements/columns from $\mathbf{A}$ to get an $n \times(m-\ell)$ matrix $\mathbf{A}^{\prime}$ and let $\mathbf{x}^{\prime}$ be the corresponding solution vector in $\mathbb{Z}^{m-\ell}$. Then solve the problem $\mathbf{A}^{\prime} \mathbf{x}^{\prime}=\mathbf{s}$ in $G$. Since the number of possible targets remains the same while the number of inputs $\mathbf{x}^{\prime}$ is reduced to $(\# \mathcal{B})^{m-\ell}$, the exponential density of the new problem is $\frac{(\# \mathcal{B})^{m-\ell}}{\# G} \approx 1$. When evaluating algorithms for high density ISIS we must always compare them against the best low-density algorithms when applied to the reduced problem.

\subsection{The Merge Algorithm}

We now introduce the notation to be used throughout. Let $\mathcal{X} \subseteq \mathcal{B}^{m}$ be a set of coefficients. We will always be working with a set of subgroups $\left\{H_{i}: 1 \leq i \leq t\right\}$ of $G$ such that, for each pair $1 \leq i<j \leq t$ we have $\#\left(G /\left(H_{i} \cap H_{j}\right)\right)=\#\left(G / H_{i}\right) \cdot \#\left(G / H_{j}\right)$. All algorithms involve splitting the set of coefficients $\mathcal{X} \subseteq \mathcal{X}_{1}+\mathcal{X}_{2}=\left\{\mathbf{x}_{1}+\mathbf{x}_{2}: \mathbf{x}_{1} \in \mathcal{X}_{1}, \mathbf{x}_{2} \in \mathcal{X}_{2}\right\}$ in some way (for example, by positions or by weight).

We consider one step of the merge algorithm. ${ }^{3}$ Let $H^{\mathrm{b}}, H, H^{\sharp}$ be subgroups of $G$ that denote subgroups used in the CPW/HGJ/BCJ algorithms. We are merging modulo $H$ a pair of lists $L_{1}$ and $L_{2}$ that are "partial solutions" modulo $H^{b}$. In other words, the output is a set of solutions to the problem $\mathbf{A x} \equiv \mathbf{s}\left(\bmod H \cap H^{b}\right)$ for $\mathbf{x} \in \mathcal{X}$. For future processing, the output includes information about $\mathbf{A x}\left(\bmod H^{\sharp}\right)$. The details are given as Algorithm 1.

The running time of the algorithm depends on the cost of sorting/hashing $L_{2}$ and searching $\mathbf{v}$ in $L_{2}$ for every $\mathbf{u}$ in $L_{1}$, which is for example, $O\left(\# L_{2} \log _{2}\left(\# L_{2}\right)+\# L_{1} \log _{2}\right.$ $\left.\left(\# L_{2}\right)\right)$, i.e., $\tilde{O}\left(\max \left(\# L_{1}, \# L_{2}\right)\right)$. However, the time is often dominated by the total number of pairs $\left(\mathbf{x}_{1}, \mathbf{x}_{2}\right)$ considered in the algorithm, and this depends on how many values $\mathbf{u}$ give rise to matches between the two lists $L_{1}$ and $L_{2}$. Assuming the function from $\mathcal{X}$ to $G / H$ given by $\mathbf{x} \mapsto \mathbf{A x}(\bmod H)$ is regular, the total number of $\left(\mathbf{x}_{1}, \mathbf{x}_{2}\right)$ pairs such that $\mathbf{A}\left(\mathbf{x}_{1}+\mathbf{x}_{2}\right) \equiv \mathbf{s}(\bmod H)$ can be approximated by $\# L_{1} \cdot \# L_{2} / \#(G / H)$. Hence, the

\footnotetext{
${ }^{3}$ The word "merge" is not really appropriate as we are not computing a union or intersection of lists, but forming sums $\mathbf{x}_{1}+\mathbf{x}_{2}$ where $\mathbf{x}_{1} \in L_{1}$ and $\mathbf{x}_{2} \in L_{2}$. However, it is the name used by several previous authors so we continue to use it. The meaning should be clear in the context.
} 


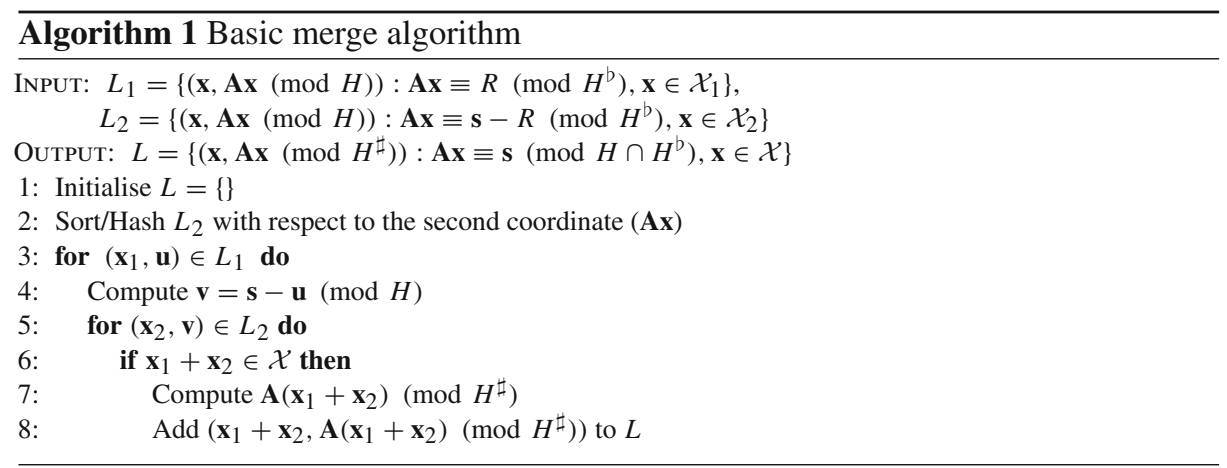

heuristic running time is $\tilde{O}\left(\max \left\{\# L_{1}, \# L_{2}, \# L_{1} \# L_{2} / \#(G / H)\right\}\right)$. (This analysis includes the correction by May and Meurer to the analysis in [15], as mentioned in Section 2.2 of [4].)

Another remark is that, in many cases, it is non-trivial to determine the size of the output list $L$. Instead, this can be heuristically estimated by $\# \mathcal{X} / \#\left(G /\left(H \cap H^{b}\right)\right)$.

\subsection{Schroeppel and Shamir Algorithm}

Schroeppel and Shamir [26] noted that by using 4 lists instead of 2 one could get an algorithm for subset-sum over $\mathbb{Z}$ with running time $(\# \mathcal{B})^{m / 2}$ but with storage growing proportional to $(\# \mathcal{B})^{m / 4}$. (Their presentation is more general than just subset-sum over $\mathbb{Z}$.)

Howgrave-Graham and Joux obtained this result in a much simpler way by using reduction modulo $M$ and Algorithm 1. Our insight is to interpret reduction modulo $M$ as working in a quotient group $G / H$. It immediately follows that the HGJ formulation of the Schroeppel-Shamir algorithm is applicable to the $(G, m, \mathcal{B})$-ISIS problem, giving an algorithm that requires time proportional to $(\# \mathcal{B})^{m / 2}$ and space proportional to $(\# \mathcal{B})^{m / 4}$. Since our goal is to discuss improved algorithms, we do not give the details here.

Dinur, Dunkelman, Keller and Shamir [11] have given improvements to the SchroeppelShamir algorithm, in the sense of getting a better time-memory curve. However, their methods always require time at least $(\# \mathcal{B})^{m / 2}$. Since we are primarily concerned with reducing the average running time, we do not consider their results further.

\subsection{Camion and Patarin/Wagner Algorithm $(C P W)$}

The CPW algorithm is applicable for instances of very high density. It splits the solution space into sets of vectors of shorter length and solves the split sub-problems. It was first proposed by Camion and Patarin for subset-sum, and then by Wagner in the additive group $\mathbb{Z}_{2}^{m}$ (and some other settings).

Section 3 of Micciancio and Regev [24] notes that the algorithm can be used to solve (I)SIS. We will describe the algorithm for the general $(G, m, \mathcal{B})$-ISIS problem.

Let $k=2^{t}$ be a small integer such that $k \mid m$. Let $H_{1}, \cdots, H_{t}$ be subgroups of the abelian group $G$ such that 


$$
G \cong\left(G / H_{1}\right) \oplus \cdots \oplus\left(G / H_{t}\right)
$$

Precisely we need that $G /\left(H_{i_{1}} \cap H_{i_{2}}\right) \cong\left(G / H_{i_{1}}\right) \oplus\left(G / H_{i_{2}}\right)$ for any $1 \leq i_{1}<i_{2} \leq t$ and $H_{1} \cap \cdots \cap H_{t}=\{0\}$. One can think of this as being like a "Chinese remainder theorem" for $G$ : there is a one-to-one correspondence between $G$ and the set of $t$-tuples $\left(g\left(\bmod H_{1}\right), \ldots, g\left(\bmod H_{t}\right)\right)$. In this subsection, we take that $\#\left(G / H_{i}\right)$ is roughly $(\# G)^{1 /(t+1)}$ for $1 \leq i<t$ and $\#\left(G / H_{t}\right) \approx(\# G)^{2 /(t+1)}$. The work of Minder and Sinclair [25] obtains improvements over the CPW algorithm by relaxing these conditions.

We now give specific examples of such subgroups for the case $G=\mathbb{Z}_{q}^{n}$. Let $\ell \in \mathbb{N}$ be such that $\ell \approx n /(t+1)$. Then we choose the subgroup $H_{1}=\left\{\left(0, \ldots, 0, g_{\ell+1}, \ldots, g_{n}\right)^{T}\right.$ : $g_{i} \in \mathbb{Z}_{q}$ \} such that $G / H_{1} \cong \mathbb{Z}_{q}^{\ell}$ corresponds to truncating the first $\ell$ positions of the vector. Similarly, $G / H_{2}$ corresponds to the next $\ell$ positions of the vector (so $\mathrm{H}_{2}=$ $\left.\left\{\left(g_{1}, \ldots, g_{\ell}, 0, \ldots, 0, g_{2 \ell+1}, \ldots, g_{n}\right)^{T}: g_{i} \in \mathbb{Z}_{q}\right\}\right)$. Finally, $G / H_{t}$ corresponds to the last $\approx 2 \ell$ positions of the vector. The "splitting" in the CPW approach is by positions. To be precise, let $u=m / k$ and define $\mathcal{X}_{1}=\left\{\left(x_{1}, \ldots, x_{u}, 0, \ldots, 0\right) \in \mathcal{B}^{m}\right\}$ and for $2 \leq j \leq k$ define

$$
\mathcal{X}_{j}=\left\{\left(0, \ldots, 0, x_{(j-1) u+1}, \ldots, x_{j u}, 0, \ldots, 0\right) \in \mathcal{B}^{m}\right\}
$$

Level 0: The CPW algorithm works by first constructing $k=2^{t}$ lists $L_{j}^{(0)}=\{(\mathbf{x}, \mathbf{A x}$ $\left.\left.\left(\bmod H_{1}\right)\right): \mathbf{x} \in \mathcal{X}_{j}\right\}$ for $1 \leq j \leq k-1$ and $L_{k}^{(0)}=\left\{\left(\mathbf{x}, \mathbf{A x}-\mathbf{s}\left(\bmod H_{1}\right)\right): \mathbf{x} \in \mathcal{X}_{k}\right\}$. Each list consists of $\# \mathcal{X}_{j}=(\# \mathcal{B})^{u}$ elements and can be computed in $O\left((\# \mathcal{B})^{u}\right)=$ $O\left((\# \mathcal{B})^{m / 2^{t}}\right)$ operations in $G$. (To optimize the running time one only computes $\mathbf{A x}$ (mod $\left.H_{1}\right)$ at this stage.)

Level 1: Use Algorithm 1 to merge the lists from level 0 to compute the $k / 2$ new lists $L_{1}^{(1)}, \ldots, L_{k / 2}^{(1)}$. Note that for $1 \leq j \leq k / 2-1$ each element of $L_{j}^{(1)}$ corresponds to a pair $\left(\mathbf{x}_{1}, \mathbf{x}_{2}\right) \in L_{2 j-1}^{(0)} \times L_{2 j}^{(0)}$ such that $\mathbf{A}\left(\mathbf{x}_{1}+\mathbf{x}_{2}\right) \equiv \mathbf{0}\left(\bmod H_{1}\right)$ and $L_{k / 2}^{(1)}$ contains pairs $\left(\mathbf{x}_{1}, \mathbf{x}_{2}\right) \in L_{k-1}^{(0)} \times L_{k}^{(0)}$ such that $\mathbf{A}\left(\mathbf{x}_{1}+\mathbf{x}_{2}\right)-\mathbf{s} \equiv \mathbf{0}\left(\bmod H_{1}\right)$. In other words, the new lists $L_{j}^{(1)}$ for $1 \leq j \leq k / 2$ contain elements $\mathbf{x}_{1}+\mathbf{x}_{2}$ that are "correct" for the quotient $G / H_{1}$. To optimize the running time one only computes $\mathbf{A}\left(\mathbf{x}_{1}+\mathbf{x}_{2}\right)\left(\bmod H_{2}\right)$ when working at this level, rather than the full values $\mathbf{A}\left(\mathbf{x}_{1}+\mathbf{x}_{2}\right) \in G$. The output of the algorithm is $k / 2$ new lists, for $1 \leq j \leq k / 2-1, L_{j}^{(1)}=\left\{\left(\mathbf{x}_{1}+\mathbf{x}_{2}, \mathbf{A}\left(\mathbf{x}_{1}+\mathbf{x}_{2}\right)\right.\right.$ $\left.\left.\left(\bmod H_{2}\right)\right): \mathbf{A}\left(\mathbf{x}_{1}+\mathbf{x}_{2}\right) \equiv \mathbf{0}\left(\bmod H_{1}\right), \mathbf{x}_{1} \in \mathcal{X}_{2 j-1}, \mathbf{x}_{2} \in \mathcal{X}_{2 j}\right\}$, and $L_{k / 2}^{(1)}=\left\{\left(\mathbf{x}_{1}+\right.\right.$ $\left.\left.\mathbf{x}_{2}, \mathbf{A}\left(\mathbf{x}_{1}+\mathbf{x}_{2}\right)\left(\bmod H_{2}\right)\right): \mathbf{A}\left(\mathbf{x}_{1}+\mathbf{x}_{2}\right)-\mathbf{s} \equiv \mathbf{0}\left(\bmod H_{1}\right), \mathbf{x}_{1} \in \mathcal{X}_{k-1}, \mathbf{x}_{2} \in \mathcal{X}_{k}\right\}$.

Level $i \geq 2$ : Use Algorithm 1 to merge the lists $L_{2 j-1}^{(i-1)}$ and $L_{2 j}^{(i-1)}$ from level $i-1$. The output of the algorithm is $k / 2^{i}$ lists $L_{j}^{(i)}$ containing elements that are "correct" for the quotient $G /\left(H_{1} \cap \cdots \cap H_{i}\right)$. Precisely, for $1 \leq j \leq k / 2^{i}-1, L_{j}^{(i)}=\left\{\left(\mathbf{x}_{1}+\right.\right.$ $\left.\left.\mathbf{x}_{2}, \mathbf{A}\left(\mathbf{x}_{1}+\mathbf{x}_{2}\right)\left(\bmod H_{i+1}\right)\right): \mathbf{A}\left(\mathbf{x}_{1}+\mathbf{x}_{2}\right) \equiv \mathbf{0}\left(\bmod H_{i}\right), \mathbf{x}_{1} \in L_{2 j-1}^{(i-1)}, \mathbf{x}_{2} \in L_{2 j}^{(i-1)}\right\}$ 
and $L_{k / 2^{i}}^{(i)}=\left\{\left(\mathbf{x}_{1}+\mathbf{x}_{2}, \mathbf{A}\left(\mathbf{x}_{1}+\mathbf{x}_{2}\right)\left(\bmod H_{i+1}\right)\right): \mathbf{A}\left(\mathbf{x}_{1}+\mathbf{x}_{2}\right)-\mathbf{s} \equiv \mathbf{0}\left(\bmod H_{i}\right), \mathbf{x}_{1} \in\right.$ $\left.L_{k / 2^{i-1}-1}^{(i-1)}, \mathbf{x}_{2} \in L_{k / 2^{i-1}}^{(i-1)}\right\}$.

Level $t$ : Merge the two lists $L_{1}^{(t-1)}$ and $L_{2}^{(t-1)}$ to get one list $L_{1}^{(t)}$ by ensuring the solutions are correct modulo $H_{t}$. In other words, the list contains elements that are "correct" for $G /\left(H_{1} \cap \cdots \cap H_{t}\right)=G$. The output of Algorithm 1 at this stage is a list

$$
\begin{aligned}
L_{1}^{(t)} & =\left\{\mathbf{x}_{1}+\mathbf{x}_{2}: \mathbf{A}\left(\mathbf{x}_{1}+\mathbf{x}_{2}\right)-\mathbf{s} \equiv \mathbf{0} \quad\left(\bmod H_{t}\right), \mathbf{x}_{1} \in L_{1}^{(t-1)}, \mathbf{x}_{2} \in L_{2}^{(t-1)}\right\} \\
& =\left\{\mathbf{x} \in \mathcal{B}^{m}: \mathbf{A x}=\mathbf{s}\right\} .
\end{aligned}
$$

Success Probability. Note that the algorithm is deterministic, but we can consider the probability over uniformly chosen problem instances $(A, \mathbf{s})$ that it gives a correct result. The heuristic analysis of the success probability is based on analyzing the expected size of lists in the algorithm: if $L_{1}^{(t)}$ is not empty, the CPW algorithm succeeds to find a solution for (I)SIS. We rely on the following heuristic, which is a stronger version of the regularity of knapsack functions (see Section 4.1 of Micciancio [22] or Ajtai [2]).

Heuristic 1. We may model the elements $\boldsymbol{A x}\left(\bmod H_{i}\right)\left(\right.$ and $\left.\boldsymbol{A x}-\boldsymbol{s}\left(\bmod H_{i}\right)\right)$ in the lists for merging as uniformly and independently sampled from $G / H_{i}$.

Assuming this heuristic, the expected size of the lists on each level is (omitting subscripts)

$$
\begin{aligned}
& \# L^{(0)} \approx(\# \mathcal{B})^{m / 2^{t}}, \\
& \# L^{(i)} \approx \frac{\# L^{(i-1)} \cdot \# L^{(i-1)}}{q^{\ell}} \text { for } 1 \leq i \leq t-1, \\
& \# L^{(t)} \approx \frac{\# L^{(t-1)} \cdot \# L^{(t-1)}}{q^{2 \ell}} .
\end{aligned}
$$

To have $\# L_{1}^{(t)} \approx 1$, the standard argument is that we want the lists $L^{(1)}, \ldots, L^{(t-1)}$ to all be roughly the same size. It follows that we desire $\ell \approx n /(t+1),(\# \mathcal{B})^{2 m / k} /(\# G)^{1 /(t+1)} \approx$ $(\# \mathcal{B})^{m / k}$ and so $(\# G)^{1 /(t+1)} \approx(\# \mathcal{B})^{m / k}$ (i.e., $\left.2^{t} /(t+1) \approx \log _{2}\left((\# \mathcal{B})^{m}\right) / \log _{2}(\# G)\right)$. Then the final list at level $t$ has expected size $\approx 1$. We refer to $[8,21,24,25,28]$ for full details and heuristic analysis.

Lyubashevsky [20] and Minder and Sinclair [25] provide some rigorous analysis of the success probability (over random instances) of the CPW algorithm that supports the validity of the heuristic analysis.

Running Time. In practice for a given (I)SIS instance, the parameters $(\# \mathcal{B}),(\# G)$ and $m$ are given. One takes $k=2^{t}$ to be as large as possible subject to the constraint $(\# \mathcal{B})^{m / 2^{t}} \geq(\# G)^{1 /(t+1)}$; in other words $t$ is the largest integer such that $2^{t} /(t+1) \leq$ $\log _{2}\left((\# \mathcal{B})^{m}\right) / \log _{2}(\# G)$. Hence the size of $k$ is governed by the density of the instance (higher density means larger $k$ ). When the density is 1 (i.e., $(\# \mathcal{B})^{m} \approx(\# G)$ ) then we 
need to have $k=1+\log _{2}(k)$ and hence $k=2$, and the CPW algorithm becomes the trivial "meet-in-the-middle" method.

Lowering the Density. Assume that, for some integer $t$, the exponential density for the (I)SIS instance (where $G=\mathbb{Z}_{q}^{n}$ ) satisfies the constraint:

$$
\frac{2^{t-1}}{t}<\frac{\log _{2}\left((\# \mathcal{B})^{m}\right)}{\log _{2}\left(q^{n}\right)}<\frac{2^{t}}{t+1}
$$

since $\frac{\log _{2}\left((\# \mathcal{B})^{m}\right)}{\log _{2}\left(q^{n}\right)}<\frac{2^{t}}{t+1}$ the largest $k$ we can choose is $2^{t-1}$. Directly using the CPW algorithm the time complexity is $\tilde{O}\left(2^{t-1} \cdot(\# \mathcal{B})^{m / 2^{t-1}}\right)$. However, since $\frac{2^{t-1}}{t}<\frac{\log _{2}\left((\# \mathcal{B})^{m}\right)}{\log _{2}\left(q^{n}\right)}$, the density is higher than what CPW using $2^{t-1}$ lists needs to find a single solution. Hence, one can lower the density: Choose an integer $\ell_{0}$ such that $\frac{\log _{2}\left((\# \mathcal{B})^{m-\ell_{0}}\right)}{\log _{2}\left(q^{n}\right)} \approx \frac{2^{t-1}}{t}$. In other words, $(\# \mathcal{B})^{\left(m-\ell_{0}\right) / 2^{t-1}} \approx q^{n / t}$. Then set $\ell_{0}$ entries of $\mathbf{x}$ to be zero. In other words, delete the corresponding $\ell_{0}$ columns of $\mathbf{A}$ to get an $n \times\left(m-\ell_{0}\right)$ matrix $\mathbf{A}^{\prime}$ and let $\mathbf{x}^{\prime}$ be the corresponding vector in $\mathbb{Z}^{m-\ell_{0}}$. One can use the CPW algorithm with $k=2^{t-1}$ to solve $\mathbf{A}^{\prime} \mathbf{x}^{\prime}=\mathbf{s}$ in $G$. The time complexity is reduced to $\tilde{O}\left(2^{t-1} \cdot(\# \mathcal{B})^{\left(m-\ell_{0}\right) / 2^{t-1}}\right)=\tilde{O}\left(2^{t-1} \cdot q^{n / t}\right)$.

We will see later that Minder and Sinclair's method works better for such a situation (when reducing the density is required). However when combined with other strategies such as the HNF, it does not rule out the possibility of using the above idea. In particular, a combination of these strategies may lead to a better algorithm (see for example Sect. 5.1).

Remark 2. The main drawbacks of the CPW algorithm are: it requires very large storage (the time and memory complexity are approximately equal); it is not amenable to parallelization; it can only be used for very high density instances. Some techniques to reduce storage and benefit from parallelism are given by Bernstein et al $[5,6]$. Note that the algorithm is completely deterministic, and so always gives the same solution set, but to obtain different solutions one can apply a random permutation to the problem before running the algorithm.

\subsection{Minder and Sinclair Refinement of $C P W$}

In this subsection, we recall the work of Minder and Sinclair [25] that allows a finer balancing of parameters and extends the range of the CPW algorithm to larger values of $k=2^{t}$ than the density of the (I)SIS instance might predict. Assume the density for a (I)SIS instance with $G=\mathbb{Z}_{q}^{n}$ satisfies the constraint in Equation (3) for some integer $t$. This implies \# $\mathcal{B}^{m / 2^{t}}<q^{n /(t+1)}<q^{n / t}<\# \mathcal{B}^{m / 2^{t-1}}$.

Instead of lowering the density as described in the previous subsection, Minder and Sinclair proposed the "extended $k$-tree" algorithm to make use of the extra density. When the density satisfies Equation (3), Minder and Sinclair use $k=2^{t}$ by choosing appropriate subgroups $H_{i}$. Note in the previous subsection, the CPW algorithm chooses $k=2^{t-1}$. 
Let $\ell_{i} \geq 1$ be chosen later, subject to $\ell_{1}+\ell_{2}+\cdots+\ell_{t}=n$. The subgroup $H_{1}=$ $\left\{\left(0, \ldots, 0, g_{\ell_{1}+1}, \ldots, g_{n}\right)^{T}: g_{i} \in \mathbb{Z}_{q}\right\}$ such that $G / H_{1} \cong \mathbb{Z}_{q}^{\ell_{1}}$ corresponds to the first $\ell_{1}$ positions of the vector. Similarly, $H_{2}=\left\{\left(g_{1}, \ldots, g_{\ell_{1}}, 0, \ldots, 0, g_{\ell_{1}+\ell_{2}+1}, \ldots, g_{n}\right)\right.$ : $\left.g_{i} \in \mathbb{Z}_{q}\right\}$ satisfies $G / H_{2} \cong \mathbb{Z}_{q}^{\ell_{2}}$ and corresponds to the next $\ell_{2}$ positions of the vector. Finally, $H_{t}=\left\{\left(g_{1}, \ldots, g_{\ell_{1}+\cdots+\ell_{t-1}}, 0, \ldots, 0\right): g_{i} \in \mathbb{Z}_{q}\right\}$ corresponds to the last $\ell_{t}$ positions of the vector. Denote by $L^{(i)}$ any of the lists at the $i$ th stage of the algorithm (the lists at the same level have the same size). The heuristic time complexity for Minder and Sinclair's algorithm is $\tilde{O}\left(2^{t} \cdot \max _{0 \leq i \leq t}\left(\# L^{(i)}\right)\right)$. To minimize the running time, one needs to minimize $\max _{0 \leq i \leq t}\left(\# L^{(i)}\right)$. The expected size of the lists on each level is

$$
\begin{aligned}
\# L^{(0)} & =(\# \mathcal{B})^{m / 2^{t}}, \\
\# L^{(i)} & =\frac{\# L^{(i-1)} \cdot \# L^{(i-1)}}{q^{\ell_{i}}} \text { for } 1 \leq i \leq t .
\end{aligned}
$$

Write $\# L^{(i)}=2^{b_{i}}$ where $b_{0}=m \log _{2}(\# \mathcal{B}) / 2^{t}, b_{i}=2 b_{i-1}-\log _{2}(q) \ell_{i}$. To minimize the time complexity, one computes the optimal values $\ell_{i}$ by solving the following integer programming problem.

$$
\begin{array}{ll}
\text { Minimize } & b_{\max }=\max _{0 \leq i \leq t} b_{i} . \\
\text { subject to } & 0 \leq b_{i} \leq b_{\max } \text { for } 0 \leq i \leq t, \\
& b_{0}=m \log _{2}(\# \mathcal{B}) / 2^{t}, \\
& b_{i}=2 b_{i-1}-\ell_{i} \log _{2}(q) \text { for } 1 \leq i \leq t, \\
& \ell_{i} \geq 0 \text { for } 1 \leq i \leq t, \\
& \sum_{i=1}^{t} \ell_{i}=n .
\end{array}
$$

Theorem 3.1 in [25] shows that the solution to the above linear program (i.e., ignoring the constraint $\left.\ell_{i} \in \mathbb{Z}\right)$ is $\ell_{2}=\cdots=\ell_{t-1}=\left(n-\ell_{1}\right) /(t+1)$ and $\ell_{t}=2\left(n-\ell_{1}\right) /(t+1)$ where $\ell_{1}$ satisfies

$$
\frac{(\# \mathcal{B})^{m / 2^{t}} \cdot(\# \mathcal{B})^{m / 2^{t}}}{q^{\ell_{1}}}=q^{\left(n-\ell_{1}\right) /(t+1)} .
$$

This gives $\max _{0 \leq i \leq t}\left(\# L^{(i)}\right)=q^{\left(n-\ell_{1}\right) /(t+1)}$. From Eq. (3), we have $0<\ell_{1}<n /(t+1)$. The time complexity of Minder and Sinclair's algorithm ${ }^{4}$ is $\tilde{O}\left(2^{t} \cdot \max _{0 \leq i \leq t}\left(\# L^{(i)}\right)\right)=$ $\tilde{O}\left(2^{t} \cdot q^{\left(n-\ell_{1}\right) /(t+1)}\right)$. It follows that

$$
(\# \mathcal{B})^{m / 2^{t}}<\max _{0 \leq i \leq t}\left(\# L^{(i)}\right)=q^{\left(n-\ell_{1}\right) /(t+1)}<(\# \mathcal{B})^{m / 2^{t-1}}
$$

\footnotetext{
${ }^{4}$ In practice, we want $\ell_{i}$ to be integers, so good parameter choices can be obtained by using $\left\lceil\ell_{i}\right\rceil$, see [25] for details. The time complexity is therefore a little bit worse, however this rounding does not affect the asymptotic complexity.
} 
The time complexity for Minder and Sinclair's refinement of CPW is better than the methods described in the previous subsection since $q^{\left(n-\ell_{1}\right) / t}<(\# \mathcal{B})^{m / 2^{t-1}}$ (not reducing the density) and $q^{\left(n-\ell_{1}\right) / t}<q^{n / t}$ (after reducing the density). At a high level, Minder and Sinclair make use of the extra density in the instance to add one more level that eliminates $\ell_{1}$ coordinates.

\subsection{The Algorithm of Howgrave-Graham and Joux (HGJ)}

We now present the HGJ algorithm, that can be applied for instances of the $(G, m, \mathcal{B})$ ISIS problem of density $\leq 1$. The algorithm heuristically improves on the square-root time complexity of Schroeppel-Shamir. For simplicity we focus on the case $\mathcal{B}=\{0,1\}$. Section 6 of [15] notes that a possible extension is to develop the algorithm for "vectorial knapsack problems". Our formulation contains this predicted extension.

The first crucial idea of Howgrave-Graham and Joux [15] is to split the vector $\mathbf{x}$ by weight rather than by positions. The second crucial idea is to reduce to a simpler problem and then apply the algorithm recursively. The procedures in [15] use reduction modulo $M$, which we generalize as a map into a quotient group $G / H$. It follows that the HGJ algorithm can be expressed in terms of our general framework.

Suppose $\mathbf{s}=\mathbf{A x}$ in $G$ where $\mathbf{x} \in \mathcal{B}^{m}$ has weight wt $(\mathbf{x})=\omega$. Our goal is to compute $\mathbf{x}$. Write $\mathcal{X}$ for the set of weight $\omega$ vectors in $\mathcal{B}^{m}$, and write $\mathcal{X}_{1}, \mathcal{X}_{2}$ for the set of weight $\omega / 2$ vectors in $\mathcal{B}^{m}$. Then there are $\left(\begin{array}{c}\omega \\ \omega / 2\end{array}\right)$ ways to write $\mathbf{x}$ as $\mathbf{x}_{1}+\mathbf{x}_{2}$ where $\mathbf{x}_{1} \in \mathcal{X}_{1}, \mathbf{x}_{2} \in \mathcal{X}_{2}$.

The procedure is to choose a suitable subgroup $H$ so that there is a good chance that a randomly chosen element $R \in G / H$ can be written as $\mathbf{A} \mathbf{x}_{1}$ for one of the $\left(\begin{array}{c}\omega \\ \omega / 2\end{array}\right)$ choices for $\mathbf{x}_{1}$. Then the procedure solves the two subset-sum instances in the group $G / H$ (recursively) to generate lists of solutions

$$
L_{1}=\left\{\mathbf{x}_{1} \in \mathcal{B}^{m}: \mathbf{A} \mathbf{x}_{1}=R \quad(\bmod H), \operatorname{wt}\left(\mathbf{x}_{1}\right)=\omega / 2\right\}
$$

and

$$
L_{2}=\left\{\mathbf{x}_{2} \in \mathcal{B}^{m}: \mathbf{A} \mathbf{x}_{2}=\mathbf{s}-R \quad(\bmod H), \operatorname{wt}\left(\mathbf{x}_{2}\right)=\omega / 2\right\} .
$$

We actually store pairs of values $\left(\mathbf{x}_{1}, \mathbf{A} \mathbf{x}_{1}\left(\bmod H^{\sharp}\right)\right) \in \mathcal{B}^{m} \times\left(G / H^{\sharp}\right)$ for a suitably chosen subgroup $H^{\sharp}$. One then applies Algorithm 1 to merge the lists to get solutions $\mathbf{x}=\mathbf{x}_{1}+\mathbf{x}_{2} \in \mathcal{X}$ satisfying the equation in $G /\left(H \cap H^{\sharp}\right)$. The paper [15] gives several solutions to the problem of merging lists, including a 4-list merge. But the main algorithm in [15] exploits Algorithm 1.

The subgroup $H$ is chosen to trade-off the probability that a random value $R$ corresponds to some splitting of the desired original solution $\mathbf{x}$ (this depends on the size of the quotient group $G / H$ ), while also ensuring that the lists $L_{1}$ and $L_{2}$ are not too large.

The improvement in complexity for finding the solutions in $L_{1}$ and $L_{2}$ is due to the lowering of the weight from $\omega$ to $\omega / 2$. This is why the process is amenable to recursive solution. At some point one terminates the recursion and solves the problem by a more elementary method (e.g., Schroeppel-Shamir).

One inconvenience is that we may not know exactly the weight of the desired solution $\mathbf{x}$. If we can guess that the weight of $\mathbf{x}$ lies in $[\omega-2 \epsilon, \omega+2 \epsilon]$ then we can construct lists 
$\left\{\mathbf{x}_{1}: \mathbf{A} \mathbf{x}_{1}=R(\bmod H), \operatorname{wt}\left(\mathbf{x}_{1}\right) \in[\omega / 2-\epsilon, \omega / 2+\epsilon]\right\}$. A similar idea can be used at the bottom level of the recursion, when we apply the Schroeppel-Shamir method and so need to split into vectors of half length and approximately half the weight.

One must pay attention to the relationship between the size of the group $G / H$ and the original group $G$. For example, when solving modular subset-sum in $G=\mathbb{Z}_{q}$ where $q$ does not have factors of a suitable size then, as noted in [15], "we first need to transform the problems into (polynomially many instances of) integer knapsacks". For the case $G=\mathbb{Z}_{q}^{n}$ this should not be necessary.

Heuristic Complexity Analysis. The final algorithm is a careful combination of these procedures, performed recursively. We limit our discussion to recursion of 3 levels. In terms of the subgroups, the recursive nature of the algorithm requires a sequence of subgroups $\mathrm{H}_{1}, \mathrm{H}_{2}, \mathrm{H}_{3}$ (of similar form to those in Sect. 2.5, but we now require $\left.H_{1} \cap H_{2} \cap H_{3} \neq\{0\}\right)$ so that the quotient groups $G /\left(H_{1} \cap H_{2} \cap H_{3}\right), G /\left(H_{2} \cap H_{3}\right)$, $G / H_{3}$ become smaller and smaller. The "top level" of the recursion turns an ISIS instance in $G$ to two lower-weight ISIS instances in $G^{\prime}=G /\left(H_{1} \cap H_{2} \cap H_{3}\right)$; to solve these sub-instances using the same method we need to choose a quotient of $G^{\prime}$ by some proper subgroup $H_{2} \cap H_{3}$, which is the same as taking a quotient of $G$ by the subgroup $\mathrm{H}_{2} \cap \mathrm{H}_{3}$ etc.

In [15], for subset-sum over $\mathbb{Z}$, this tower of subgroups is manifested by taking moduli $M$ that divide one another ("For the higher level modulus, we choose $M=4194319$. $58711 \cdot 613$ ", meaning $H_{3}=613 \mathbb{Z}, H_{2}=58711 \mathbb{Z}, H_{1}=4194319 \mathbb{Z}, H_{2} \cap H_{3}=$ $(58711 \cdot 613) \mathbb{Z}$ and $\left.H_{1} \cap H_{2} \cap H_{3}=M \mathbb{Z}\right)$.

We do not reproduce all the analysis from [15], since it is superseded by the method of Becker et al. But the crucial aspect is that the success of the algorithm depends on the probability that there is a splitting $\mathbf{x}=\mathbf{x}_{1}+\mathbf{x}_{2}$ of the solution into equal weight terms such that $\mathbf{A} \mathbf{x}_{1}=R(\bmod H)$. This depends on the number $\left(\begin{array}{c}\omega \\ \omega / 2\end{array}\right)$ of splittings of the weight $\omega$ vector $\mathbf{x}$ and on the size $M=\#(G / H)$ of the quotient group. Overall, the heuristic running time for the HGJ method to solve the (I)SIS problem when $\omega=m / 2$ (as stated in Section 2.2 of [4]) is $\tilde{O}\left(2^{0.337 m}\right)$. The running-time is restated in Table 2.

\subsection{The Algorithm of Becker, Coron and Joux}

Becker, Coron and Joux [4] present an improved version of the HGJ algorithm (again, their paper is in the context of subset-sum, but we explain how it can be adapted to our more general setting). The idea is to allow larger coefficient sets. Precisely, suppose $\mathcal{B}=\{0,1\}$ and let $\mathcal{X} \subset \mathcal{B}^{m}$ be the set of weight $\omega$ vectors. The HGJ idea is to split $\mathcal{X}$ by taking $\mathcal{X}_{1}=\mathcal{X}_{2}$ to be the set of weight $\omega / 2$ vectors in $\mathcal{B}^{m}$. Becker et al suggest to take $\mathcal{X}_{1}=\mathcal{X}_{2}$ to be the set of vectors in $\mathbb{Z}^{m}$ having $\omega / 2+\alpha m$ entries equal to +1 and $\alpha m$ entries equal to -1 , and the remaining entries equal to 0 . This essentially increases the density of the sub-problems, and leads to a better choice of parameters. The organization of the algorithm, and its analysis, are the same as HGJ. The HGJ algorithm is simply the case $\alpha=0$ of the BCJ algorithm.

We briefly sketch the heuristic analysis from [4] for the case of 3 levels of recursion, $\mathcal{B}=\{0,1\}, G=\mathbb{Z}_{q}^{n}$, and where we solve ISIS instances of density 1 (so that $2^{m} \approx q^{n}$ ) with a solution of weight $m / 2$. Let 


$$
\mathcal{X}_{a, b}=\left\{\mathbf{x} \in\{-1,0,1\}^{m}: \#\left\{i: x_{i}=1\right\}=a m, \#\left\{i: x_{i}=-1\right\}=b m\right\} .
$$

A good approximation to $\# \mathcal{X}_{a, b}$ is $2^{H(a, b) \cdot m}$ where $H(x, y)=-x \log _{2}(x)-y \log _{2}(y)-$ $(1-x-y) \log _{2}(1-x-y)$.

Choose subgroups $H_{1}, H_{2}, H_{3}$ (of the similar form to Sect. 2.6) such that \# $\left(G / H_{i}\right)=$ $q^{\ell_{i}}$. Fix $\alpha=0.0267, \beta=0.0168$ and $\gamma=0.0029$ and also integers $\ell_{1}, \ell_{2}, \ell_{3}$ such that $q^{\ell_{1}} \approx q^{0.2673 n} \approx 2^{0.2673 m}, q^{\ell_{2}} \approx 2^{0.2904 m}$ and $q^{\ell_{3}} \approx 2^{0.2408 m}$. Note that $\#\left(G /\left(H_{1} \cap\right.\right.$ $\left.\left.H_{2} \cap H_{3}\right)\right) \approx q^{0.2015 n} \approx 2^{0.2015 m}$.

Theorem 1. (Becker-Coron-Joux) With notation as above, and assuming Heuristic 1 about the regularity of $\boldsymbol{A x}\left(\bmod H_{i}\right)$, the BCJ algorithm runs in time $\tilde{O}\left(2^{0.2912 m}\right)$.

Proof. (Sketch) The first level of recursion splits $\mathcal{X}=\mathcal{B}^{m}$ into $\mathcal{X}_{1}+\mathcal{X}_{2}$ where $\mathcal{X}_{1}=$ $\mathcal{X}_{2}=\mathcal{X}_{1 / 4+\alpha, \alpha}$. We choose uniformly at random $R_{1} \in G /\left(H_{1} \cap H_{2} \cap H_{3}\right)$ and compute two lists $L_{1}^{(1)}=\left\{\left(\mathbf{x}, \mathbf{A x}\left(\bmod H_{1} \cap H_{2}\right)\right): \mathbf{x} \in \mathcal{X}_{1}, \mathbf{A x} \equiv R_{1}\left(\bmod H_{1} \cap H_{2} \cap H_{3}\right)\right\}$ and $L_{2}^{(1)}$, which is the same except $\mathbf{A x} \equiv \mathbf{s}-R_{1}\left(\bmod H_{1} \cap H_{2} \cap H_{3}\right)$. By Heuristic 1 the expected size of the lists is $2^{H(1 / 4+\alpha, \alpha) m} / q^{\ell_{1}+\ell_{2}+\ell_{3}}=2^{0.2173 m}$ and merging requires $\tilde{O}\left(\left(2^{0.2173 m}\right)^{2} / q^{n-\ell_{1}-\ell_{2}-\ell_{3}}\right)=\tilde{O}\left(2^{0.2331 m}\right)$ time.

The second level of recursion computes each of $L_{1}^{(1)}$ and $L_{2}^{(1)}$, by splitting into further lists. Choose uniformly $R_{2} \in G /\left(H_{1} \cap H_{2}\right)$ and split $L_{1}^{(1)}$ into $L_{1}^{(2)}$ and $L_{2}^{(2)}$ where $L_{1}^{(2)}=\left\{(\mathbf{x}, \mathbf{A x}): \mathbf{x} \in \mathcal{X}_{1 / 8+\alpha / 2+\beta, \alpha / 2+\beta}, \mathbf{A x} \equiv R_{2}\left(\bmod H_{2} \cap H_{3}\right)\right\}$ and $L_{2}^{(2)}$ is similar except the congruence is $\mathbf{A x} \equiv R_{1}-R_{2}\left(\bmod H_{2} \cap H_{3}\right)$. We split $L_{2}^{(1)}$ into sets $L_{3}^{(2)}$ and $L_{4}^{(2)}$ a similar way using a random $R_{3} \in G /\left(H_{1} \cap H_{2}\right)$. Again, by Heuristic 1 , the size of lists is approximately $2^{H(1 / 8+\alpha / 2+\beta, \alpha / 2+\beta) m} / q^{\ell_{2}+\ell_{3}}=2^{0.2790 m}$ and the cost to merge is $\tilde{O}\left(2^{2 \cdot 0.2790 m} / q^{\ell_{1}}\right)=\tilde{O}\left(2^{(2 \cdot 0.2790-0.2673) m}\right)=\tilde{O}\left(2^{0.2907 m}\right)$.

The final level of recursion computes each $L_{j}^{(2)}$ by splitting into two lists corresponding to coefficient sets $\mathcal{X}_{1 / 16+\alpha / 4+\beta / 2+\gamma, \alpha / 4+\beta / 2+\gamma}$. The expected size of the lists is

$$
2^{H(1 / 16+\alpha / 4+\beta / 2+\gamma, \alpha / 4+\beta / 2+\gamma) m} / q^{\ell_{3}} \approx 2^{0.2908 m}
$$

and they can be computed efficiently using the Shroeppel-Shamir algorithm in time

$$
\tilde{O}\left(\sqrt{2^{H(1 / 16+\alpha / 4+\beta / 2+\gamma, \alpha / 4+\beta / 2+\gamma) m}}\right)=\tilde{O}\left(2^{0.2658 m}\right) .
$$

Merging the lists takes $\tilde{O}\left(2^{2 \cdot 0.2908} / q^{\ell_{2}}\right)=\tilde{O}\left(2^{0.2912 m}\right)$ time. Thus the time complexity of the BCJ algorithm is $\tilde{O}\left(2^{0.2912 m}\right)$.

The above theorem does not address the probability that the algorithm succeeds to output a solution to the problem. The discussion of this issue is complex and takes more than 3 pages (Section 3.4) of [4]. We give a rough "back-of-envelope" calculation that gives some confidence.

Suppose there is a unique solution $\mathbf{x} \in\{0,1\}^{m}$ of weight $m / 2$ to the ISIS instance. Consider the first step of the recursion. For the whole algorithm to succeed, it is necessary that there is a splitting $\mathbf{x}=\mathbf{x}_{1}+\mathbf{x}_{2}$ of the solution so that $\mathbf{x}_{1} \in L_{1}^{(1)}$ and $\mathbf{x}_{2} \in L_{2}^{(1)}$. We 
split $\mathbf{x}$ so that the $m / 2$ ones are equally distributed across $\mathbf{x}_{1}$ and $\mathbf{x}_{2}$, and the $m / 2$ zeroes are sometimes expanded as $(-1,+1)$ or $(+1,-1)$ pairs. We call such splittings "valid". Hence, the number of ways to split $\mathbf{x}$ in this way is

$$
\mathcal{N}_{1}=\left(\begin{array}{l}
m / 2 \\
m / 4
\end{array}\right)\left(\begin{array}{c}
m / 2 \\
\alpha m
\end{array}\right)\left(\begin{array}{c}
(1 / 2-\alpha) m \\
\alpha m
\end{array}\right)=\left(\begin{array}{l}
m / 2 \\
m / 4
\end{array}\right)\left(\begin{array}{c}
m / 2 \\
\alpha m, \alpha m,(1 / 2-2 \alpha) m
\end{array}\right)
$$

Here $\left(\begin{array}{c}n \\ a, b, c\end{array}\right)$ is the usual multinomial coefficient $n ! /(a ! b ! c !)$ where $n=a+b+c$. For randomly chosen $R_{1} \in G /\left(H_{1} \cap H_{2} \cap H_{3}\right)$, there is a good chance that a valid splitting exists if $\mathcal{N}_{1} \geq q^{\ell_{1}+\ell_{2}+\ell_{3}}$. Indeed, the heuristic expected number of valid splittings is roughly $\mathcal{N}_{1} / q^{\ell_{1}+\ell_{2}+\ell_{3}}$. Hence, we choose $\mathcal{N}_{1} \approx q^{\ell_{1}+\ell_{2}+\ell_{3}}$ to make sure a valid splitting exists at this stage with significant probability.

For the second stage we assume that we already made a good choice in the first stage, and indeed that we have $\mathcal{N}_{1} / q^{\ell_{1}+\ell_{2}+\ell_{3}}$ possible values for $\mathbf{x}_{1}$. The number of ways to further split $\mathbf{x}_{1}$ is

$$
\mathcal{N}_{2}=\left(\begin{array}{c}
(1 / 4+\alpha) m \\
(1 / 8+\alpha / 2) m
\end{array}\right)\left(\begin{array}{c}
\alpha m \\
\alpha m / 2
\end{array}\right)\left(\begin{array}{c}
(3 / 4-2 \alpha) m \\
\beta m, \beta m,(3 / 4-2 \alpha-2 \beta) m
\end{array}\right) \text {. }
$$

The heuristic total expected number of valid splittings at this stage is roughly $\left(\mathcal{N}_{1} / q^{\ell_{1}+\ell_{2}+\ell_{3}}\right)\left(\mathcal{N}_{2} / q^{\ell_{2}+\ell_{3}}\right)^{2}$. For randomly chosen $R_{2} \in G /\left(H_{2} \cap H_{3}\right)$, there is a good chance to have a valid splitting if $\left(\mathcal{N}_{1} / q^{\ell_{1}+\ell_{2}+\ell_{3}}\right)\left(\mathcal{N}_{2} / q^{\ell_{2}+\ell_{3}}\right)^{2} \geq 1$ (remember that this stage requires splitting two solutions from the first stage). Hence, we choose $\mathcal{N}_{1} \approx q^{\ell_{1}+\ell_{2}+\ell_{3}}, \mathcal{N}_{2} \approx q^{\ell_{2}+\ell_{3}}$.

In the final stage (again assuming good splittings in the second stage), the number of ways to split is

$$
\mathcal{N}_{3}=\left(\begin{array}{c}
(1 / 8+\alpha / 2+\beta) m \\
(1 / 16+\alpha / 4+\beta / 2) m
\end{array}\right)\left(\begin{array}{c}
(\beta+\alpha / 2) m \\
(\beta / 2+\alpha / 4) m
\end{array}\right)\left(\begin{array}{c}
(7 / 8-\alpha-2 \beta) m \\
\gamma m, \gamma m,(7 / 8-\alpha-2 \beta-2 \gamma) m
\end{array}\right) \text {. }
$$

The heuristic expected number of valid splittings is $\left(\mathcal{N}_{1} / q^{\ell_{1}+\ell_{2}+\ell_{3}}\right)\left(\mathcal{N}_{2} / q^{\ell_{2}+\ell_{3}}\right)^{2}$ $\left(\mathcal{N}_{3} / q^{\ell_{3}}\right)^{4}$, which we require to be $\geq 1$. Hence, we choose $\mathcal{N}_{1} \approx q^{\ell_{1}+\ell_{2}+\ell_{3}}, \mathcal{N}_{2} \approx$ $q^{\ell_{2}+\ell_{3}}, \mathcal{N}_{3} \approx q^{\ell_{3}}$. Thus, choosing $\# G / H_{3}$ close to $\mathcal{N}_{3}, \# G /\left(H_{2} \cap H_{3}\right)$ close to $\mathcal{N}_{2}$ and $\# G /\left(H_{1} \cap H_{2} \cap H_{3}\right)$ close to $\mathcal{N}_{1}$ then it is believed the success probability of the algorithm is significantly larger than 0. This argument is supported in Section 3.4 of [4] by theoretical discussions and numerical experiments. To conclude, for the algorithm to have a good chance to succeed we require

$$
\begin{aligned}
& \mathcal{N}_{1} / q^{\ell_{1}+\ell_{2}+\ell_{3}} \geq 1, \quad\left(\mathcal{N}_{1} / q^{\ell_{1}+\ell_{2}+\ell_{3}}\right)\left(\mathcal{N}_{2} / q^{\ell_{2}+\ell_{3}}\right)^{2} \geq 1, \\
& \left(\mathcal{N}_{1} / q^{\ell_{1}+\ell_{2}+\ell_{3}}\right)\left(\mathcal{N}_{2} / q^{\ell_{2}+\ell_{3}}\right)^{2}\left(\mathcal{N}_{3} / q^{\ell_{3}}\right)^{4} \geq 1
\end{aligned}
$$

\subsection{Summary}

Despite the large literature on the topic, summarized above, one sees there are only two fundamental ideas that are used by all these algorithms: 
- Reduce modulo subgroups to create higher density instances. Since the new instances have higher density one now has the option to perform methods that only find some of the possible solutions.

- Splitting solutions. Splitting can be done by length (i.e., positions) or by weight. Either way, one reduces to two "simpler" problems that can be solved recursively and then "merges" the solutions back to solutions to the original problem.

The main difference between the methods is that $\mathrm{CPW}$ requires large density to begin with, in which case splitting by positions is possible. In contrast HGJ/BCJ can be applied when the original instance has low density, in which case it is necessary to use splitting by weight in order to be able to discard some potential solutions.

\section{Analysis of HGJ/BCJ for High Density}

The CPW algorithm clearly likes high density problems. However, the analysis of the HGJ and BCJ algorithms in [4,15] is in the case of finding a specific solution (and so is relevant to the case of density at most 1). It is intuitively clear that when the density is higher (and so there is more than one possible solution), and when we only want to compute a single solution to the problem, then the success probability of the algorithm (taken over problem instances) should increase. In this section we explain that the parameters in the HGJ and BCJ algorithms can be improved when one is solving instances of density $>1$. This was anticipated in [16]: "further improvements can be obtained if, in addition, we seek one solution among many". We now give a very approximate heuristic analysis of this situation. We focus on the case $\mathcal{B}=\{0,1\}^{m}$ and $G=\mathbb{Z}_{q}^{n}$. As with previous work, we are not able to give general formulae for the running time as a function of the density, as the parameters in the algorithms depend in subtle ways on each other. Instead, we fix some reference instances, compute optimal parameters for them, and give the running times.

\subsection{Analysis}

Let the number of solutions to the original (I)SIS instance be $\mathcal{N} \geq 1$ (the exponential density is the expected value of this number). We consider at most $t$ levels of recursion. The subgroups $H_{1}, H_{2}, \cdots, H_{t}$, where $\#\left(G / H_{i}\right)=q^{\ell_{i}}$, are chosen to trade-off the probability of a successful split at each stage and also to ensure the size of the lists to be merged at each stage is not too large. Using the same notation as Sect. 2.8, write $\mathcal{N}_{1}, \mathcal{N}_{2}, \ldots, \mathcal{N}_{t}$ for the number of ways to split a single valid solution at each level of the recursion.

The standard approach is to choose the subgroups $H_{1}, H_{2}, \ldots, H_{t}$ such that \#G/( $H_{i} \cap$ $\left.H_{i+1} \cap \cdots \cap H_{t}\right)=q^{\ell_{i}+\ell_{i+1}+\cdots+\ell_{t}} \approx \mathcal{N}_{i}$ for all $1 \leq i \leq t$. The success probability is then justified by requiring

$$
\frac{\mathcal{N}_{1}}{q^{\ell_{1}+\cdots+\ell_{t}}}\left(\frac{\mathcal{N}_{2}}{q^{\ell_{2}+\cdots+\ell_{t}}}\right)^{2} \cdots\left(\frac{\mathcal{N}_{i}}{q^{\ell_{i}+\cdots+\ell_{t}}}\right)^{2^{i-1}} \geq 1
$$


Table 1. Heuristic time complexity of CPW for different densities. The exponential density is $2^{c_{1} m}$.

\begin{tabular}{lll}
\hline$t$ & $2^{c_{1} m}$ & $\tilde{O}\left(2^{c_{2} m}\right)$ \\
\hline 1 & 1 & $\tilde{O}\left(2^{0.5 m}\right)$ \\
2 & $2^{0.25 m}$ & $\tilde{O}\left(2^{0.25 m}\right)$ \\
3 & $2^{0.5 m}$ & $\tilde{O}\left(2^{0.125 m}\right)$ \\
4 & $2^{0.6875 m}$ & $\tilde{O}\left(2^{0.0625 m}\right)$ \\
\hline
\end{tabular}

for all $1 \leq i \leq t$. We now assume a best-case scenario, that all the splittings of all the $\mathcal{N} \geq 1$ solutions are distinct. This assumption is clearly unrealistic for large values of $\mathcal{N}$, but it gives a rough idea of how much speedup one can ask with this approach. We address this assumption in Sect. 3.2. Then the success condition changes, for all $1 \leq i \leq t$, to

$$
\mathcal{N} \frac{\mathcal{N}_{1}}{q^{\ell_{1}+\cdots+\ell_{t}}}\left(\frac{\mathcal{N}_{2}}{q^{\ell_{2}+\cdots+\ell_{t}}}\right)^{2} \cdots\left(\frac{\mathcal{N}_{i}}{q^{\ell_{i}+\cdots+\ell_{t}}}\right)^{2^{i-1}} \geq 1 .
$$

It follows that, for example when $t=3$, the best parameters $\ell_{1}, \ell_{2}, \ell_{3}, \alpha, \beta, \gamma$ are chosen by the following integer linear program.

$$
\begin{array}{ll}
\text { minimize } & T=\max \left(\frac{\#\left(L^{(1)}\right)^{2}}{q^{n-\ell_{1}-\ell_{2}-\ell_{3}}}, \frac{\#\left(L^{(2)}\right)^{2}}{q^{\ell_{1}}}, \frac{\#\left(L^{(3)}\right)^{2}}{q^{\ell_{2}}}, \sqrt{\# \mathcal{X}_{1 / 16+\alpha / 4+\beta / 2+\gamma, \alpha / 4+\beta / 2+\gamma}}\right) \\
\text { subject to } & \# L^{(1)}=\frac{\# \mathcal{X}_{1 / 4+\alpha, \alpha}}{q^{\ell_{1}+\ell_{2}+\ell_{3}}}, \\
& \# L^{(2)}=\frac{\# \mathcal{X}_{1 / 8+\alpha / 2+\beta, \alpha / 2+\beta}}{q^{\ell_{2}+\ell_{3}}}, \\
& \# L^{(3)}=\frac{\# \mathcal{X}_{1 / 16+\alpha / 4+\beta / 2+\gamma, \alpha / 4+\beta / 2+\gamma}}{q^{\ell_{3}}}, \\
& \text { equation }(5) \text { holds for all } 1 \leq i \leq 3 \\
& \ell_{i} \in \mathbb{N}, 1 \leq i \leq 3 \\
& \alpha, \beta, \gamma \in \mathbb{R}_{\geq 0} .
\end{array}
$$

For the ISIS problem $\mathcal{B}=\{0,1\}^{m}$ given $q$ and $n$, the exponential density (expected number of solutions) is $\mathcal{N}=2^{m} / q^{n}$. Define $c_{1}$ such that $\mathcal{N}=2^{c_{1} m}$. One can estimate the time complexity as $\tilde{O}\left(2^{c_{2} m}\right)$ by solving the above linear program and choosing the optimal parameters $\alpha, \beta, \gamma$ and $\ell_{1}, \ell_{2}, \ell_{3}$. We use LINGO 11.0 to do the optimization and obtain Tables 2 and 3 giving calculations for HGJ/BCJ. For comparison we recall in Table 1 the results of Sects. 2.5 and 2.6 on the time complexity of CPW. We draw Fig. 1, which indicates how the density affects the asymptotic complexity for $\mathrm{CPW},{ }^{5} \mathrm{HGJ}$ and BCJ.

These results demonstrate that, although CPW is the best choice for very high density instances, the HGJ/BCJ algorithms do perform better when the density is increased and

\footnotetext{
${ }^{5}$ We remark that [12] contains a similar figure regarding the complexity of the CPW algorithm.
} 
Table 2. Time complexity of HGJ for different density.

\begin{tabular}{llllll}
\hline$t$ & $2^{c_{1} m}$ & $\tilde{O}\left(2^{c_{2} m}\right)$ & $q^{\ell_{1}}$ & $q^{\ell_{2}}$ & $q^{\ell_{3}}$ \\
\hline 2 & $2^{0}=1$ & $\tilde{O}\left(2^{0.3371 m}\right)$ & $2^{0.25 m}$ & $2^{0.25 m}$ & - \\
2 & $2^{0.025 m}$ & $\tilde{O}\left(2^{0.3121 m}\right)$ & $2^{0.275 m}$ & $2^{0.25 m}$ & - \\
2 & $2^{0.045 m}$ & $\tilde{O}\left(2^{0.2928 m}\right)$ & $2^{0.2928 m}$ & $2^{0.2507 m}$ & - \\
2 & $2^{0.055 m}$ & $\tilde{O}\left(2^{0.2878 m}\right)$ & $2^{0.2878 m}$ & $2^{0.2557 m}$ & - \\
2 & $2^{0.085 m}$ & $\tilde{O}\left(2^{0.2728 m}\right)$ & $2^{0.2728 m}$ & $2^{0.2707 m}$ & - \\
3 & $2^{0.15 m}$ & $\tilde{O}\left(2^{0.2535 m}\right)$ & $2^{0.2617 m}$ & $2^{0.1711 m}$ & $2^{0.125 m}$ \\
3 & $2^{0.2 m}$ & $\tilde{O}\left(2^{0.2368 m}\right)$ & $2^{0.2617 m}$ & $2^{0.1878 m}$ & $2^{0.125 m}$ \\
3 & $2^{0.3 m}$ & $\tilde{O}\left(2^{0.2070 m}\right)$ & $2^{0.2670 m}$ & $2^{0.2070 m}$ & $2^{0.1303 m}$ \\
3 & $2^{0.5 m}$ & $\tilde{O}\left(2^{0.1887 m}\right)$ & $2^{0.2677 m}$ & $2^{0.1879 m}$ & $2^{0.1669 m}$ \\
\hline
\end{tabular}

Table 3. Time complexity of BCJ for different density.

\begin{tabular}{lllllllll}
\hline$t$ & $2^{c_{1} m}$ & $\tilde{O}\left(2^{c_{2} m}\right)$ & $q^{\ell_{1}}$ & $q^{\ell_{2}}$ & $q^{\ell_{3}}$ & $\alpha$ & $\beta$ & $\gamma$ \\
\hline 3 & $2^{0}=1$ & $\tilde{O}\left(2^{0.2912 m}\right)$ & $2^{0.2673 m}$ & $2^{0.2904 m}$ & $2^{0.2408 m}$ & 0.0267 & 0.0168 & 0.0029 \\
3 & $2^{0.025 m}$ & $\tilde{O}\left(2^{0.2829 m}\right)$ & $2^{0.2463 m}$ & $2^{0.2802 m}$ & $2^{0.2829 m}$ & 0.02578 & 0.01973 & 0.00534 \\
3 & $2^{0.045 m}$ & $\tilde{O}\left(2^{0.2794 m}\right)$ & $2^{0.2604 m}$ & $2^{0.2794 m}$ & $2^{0.2770 m}$ & 0.02818 & 0.01833 & 0.00453 \\
3 & $2^{0.055 m}$ & $\tilde{O}\left(2^{0.2765 m}\right)$ & $2^{0.2634 m}$ & $2^{0.2765 m}$ & $2^{0.2649 m}$ & 0.02651 & 0.01705 & 0.00391 \\
3 & $2^{0.085 m}$ & $\tilde{O}\left(2^{0.2579 m}\right)$ & $2^{0.2404 m}$ & $2^{0.2564 m}$ & $2^{0.1818 m}$ & 0.01082 & 0.00888 & 0.00131 \\
3 & $2^{0.15 m}$ & $\tilde{O}\left(2^{0.2499 m}\right)$ & $2^{0.2430 m}$ & $2^{0.2499 m}$ & $2^{0.1554 m}$ & 0.00102 & 0.00971 & 0.00023 \\
3 & $2^{0.2 m}$ & $\tilde{O}\left(2^{0.2357 m}\right)$ & $2^{0.2357 m}$ & $2^{0.2358 m}$ & $2^{0.2036 m}$ & 0.00231 & 0.01171 & 0.00206 \\
3 & $2^{0.3 m}$ & $\tilde{O}\left(2^{0.2070 m}\right)$ & $2^{0.2670 m}$ & $2^{0.2070 m}$ & $2^{0.1303 m}$ & 0 & 0 & 0 \\
3 & $2^{0.5 m}$ & $\tilde{O}\left(2^{0.1887 m}\right)$ & $2^{0.2677 m}$ & $2^{0.1879 m}$ & $2^{0.1669 m}$ & 0 & 0 & 0 \\
\hline
\end{tabular}

they are better than CPW for quite a large range of densities. The final rows of Table 3 show that the $\mathrm{BCJ}$ algorithm becomes exactly the HGJ algorithm (i.e., $\alpha=\beta=\gamma=0$ ) once the density is sufficiently high.

To invert the SWIFFT hash function the parameters are $\mathcal{B}=\{0,1\}^{m}, G=\mathbb{Z}_{q}^{n}$, $m=1024, q=257, n=64$ and so the expected number of solutions is $2^{0.5 m}$. Figure 1 therefore confirms that, for this problem, the CPW algorithm is the right choice. This answers the question from [15].

\subsection{Heuristic Justification of Assumptions}

The above analysis is based on the strong simplifying assumption that all the splittings of all the $\mathcal{N}=2^{c_{1} m}$ solutions are distinct, and it should not be assumed that the HGJ and BCJ algorithms perform exactly as well as our theoretical analysis suggests. However, we now give some evidence that the assumptions are reasonable when the density is moderate.

We have $\mathcal{N}=2^{c_{1} m}$ solutions $\mathbf{x} \in \mathcal{B}^{m}$ such that $\mathbf{A x}=\mathbf{s}$. We suppose all these solutions behave like independently chosen binary strings of Hamming weight very close to $m / 2$. Consider one solution $\mathbf{x}$. In the first level of the recursion, we split $\mathbf{x}=\mathbf{x}_{1}+\mathbf{x}_{2}$ where $\mathbf{x}_{1}, \mathbf{x}_{2} \in \mathcal{X}_{1 / 4+\alpha, \alpha}$. There are $\mathcal{N}_{1}$ ways to do this, where $\mathcal{N}_{1}$ is given in equation (4) 


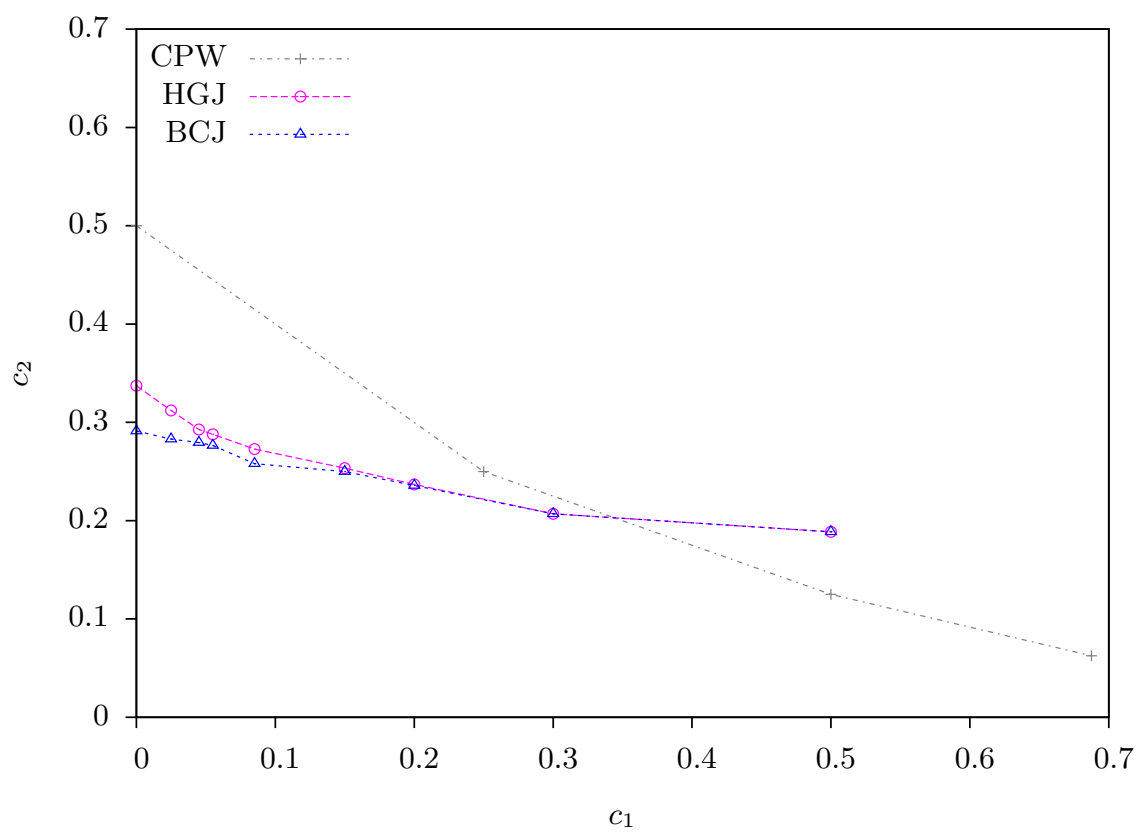

Fig. 1. Comparison of the heuristic performance of CPW, HGJ and BCJ algorithms on ISIS instances of density $\geq 1$. The horizontal axis is the value $c_{1}$ such that the expected number of solutions is $2^{c_{1} m}$. The vertical axis is the constant $c_{2}$ such that the heuristic asymptotic complexity is $\tilde{O}\left(2^{c} 2^{m}\right)$.

and all these splittings have, by definition, distinct values for $\mathbf{x}_{1}$. Turning this around, the probability that a vector $\mathbf{x}_{1} \in \mathcal{X}_{1 / 4+\alpha, \alpha}$ appears as a splitting of $\mathbf{x}$ should be $p_{1}=$ $\frac{\mathcal{N}_{1}}{\# \mathcal{X}_{1 / 4+\alpha, \alpha}}$. Now consider splitting a second solution $\mathbf{x}^{\prime}=\mathbf{x}_{1}^{\prime}+\mathbf{x}_{2}^{\prime}$. The probability that a value $\mathbf{x}_{1}^{\prime}$ is equal to one of the previous values $\mathbf{x}_{1}$ is $p_{1}$. Hence, the total number of "new" solutions $\left\{\mathbf{x}_{1}, \mathbf{x}_{1}^{\prime}\right\}$ is $\mathcal{N}_{1}+\left(1-p_{1}\right) \mathcal{N}_{1}$. The following lemma extends this argument.

Lemma 1. Let $X$ be a set. Suppose distinct subsets $X_{i}$ of $X$ of size $\mathcal{N}_{1}$ are chosen uniformly at random for $1 \leq i \leq t$. Let $p=\mathcal{N}_{1} / \# X$. Then the expected size of $\cup_{i=1}^{t} X_{i}$ is $\left(1-(1-p)^{t}\right) \mathcal{N}_{1} / p$.

Proof. The probability that any given $\mathbf{x} \in X_{1}$ lies in $X_{2}$ is $p$, so the expected size of $X_{1} \cap X_{2}$ is $p \# X_{1}$. So we expect $\#\left(X_{1} \cup X_{2}\right)=\mathcal{N}_{1}+\left(1-p_{1}\right) \mathcal{N}_{1}=(1+(1-p)) \mathcal{N}_{1}$.

The probability that any $\mathbf{x} \in X_{1} \cup X_{2}$ lies in $X_{3}$ is $p$, so the expected size of $\left(X_{1} \cup\right.$ $\left.X_{2}\right) \cap X_{3}=p \#\left(X_{1} \cup X_{2}\right)=p(1+(1-p))$. Hence, we expect \# $\left(X_{1} \cup X_{2} \cup X_{3}\right)=$ $\#\left(X_{1} \cup X_{2}\right)+\# X_{3}-\#\left(\left(X_{1} \cup X_{2}\right) \cap X_{3}\right)=(1+(1-p)+1-p(1+(1-p))) \mathcal{N}_{1}=$ $\left(1+(1-p)+(1-p)^{2}\right) \mathcal{N}_{1}$. More generally, one can show by induction that the expected value of $\#\left(X_{1} \cup \cdots \cup X_{t}\right)$ is $\left(1+(1-p)+\cdots+(1-p)^{t-1}\right) \mathcal{N}_{1}=\frac{1-(1-p)^{t}}{p} \mathcal{N}_{1}$.

Lemma 1 indicates that when we split all $\mathcal{N}$ solutions $\mathbf{x}$, the total number of vectors $\mathbf{x}_{1} \in \mathcal{X}_{1 / 4+\alpha, \alpha}$ should be roughly $\left(1-\left(1-p_{1}\right)^{\mathcal{N}}\right) \mathcal{N}_{1} / p_{1}$. If $p_{1} \mathcal{N}$ is very small $(\ll 1)$ 
then one has $\left(1-p_{1}\right)^{\mathcal{N}} \approx 1-p_{1} \mathcal{N}$ and so $\left(1-\left(1-p_{1}\right)^{\mathcal{N}}\right) / p_{1} \approx \mathcal{N}$. In other words, almost all the splittings of all the $\mathcal{N}$ solutions are distinct. For the values of $\alpha$ listed in Tables 2 and $3, \max \left(p_{1}\right)=2^{-0.2143 m}$, and so if $\mathcal{N} \leq 2^{0.2 m}$ then $p_{1} \mathcal{N} \leq 2^{-0.0143 m}$. Hence, the assumption made earlier seems justified when the number of solutions is less that $2^{0.2 m}$. Hence, for the remainder of the argument we suppose the density is low enough that our assumption made earlier holds.

In the second level of the recursion, we have $\mathcal{N}^{\prime}=\mathcal{N} \frac{\mathcal{N}_{1}}{q^{\ell_{1}+\ell_{2}+\ell_{3}}}$ solutions. Each solution has $\mathcal{N}_{2}$ splittings as a sum of vectors in $\mathcal{X}_{1 / 8+\alpha / 2+\beta, \alpha / 2+\beta}$. Let $p_{2}=\frac{\mathcal{N}_{2}}{\# \mathcal{X}_{1 / 8+\alpha / 2+\beta, \alpha / 2+\beta}}$ be the probability a random vector appears as such a splitting. Lemma 1 shows that, as long as $p_{2} \mathcal{N}^{\prime} \ll 1$, we again expect almost all the splittings to be distinct.

For all values $\alpha, \beta$ listed in Tables 2 and 3 we have $\max \left(p_{2}\right)=2^{-0.2469 m}$. Since $\mathcal{N} \frac{\mathcal{N}_{1}}{q^{\ell_{1}+\ell_{2}+\ell_{3}}} \leq \mathcal{N} \leq 2^{0.2 m}$ (when $\mathcal{N}=1$, one chooses $q^{\ell_{1}+\ell_{2}+\ell_{3}} \approx \mathcal{N}_{1}$, and if $\mathcal{N}>1$, one chooses $q^{\ell_{1}+\ell_{2}+\ell_{3}}>\mathcal{N}_{1}$ to reduce the time complexity, so $q^{\ell_{1}+\ell_{2}+\ell_{3}} \geq \mathcal{N}_{1}$ ), it follows that $p_{2} \mathcal{N}^{\prime} \leq 2^{-0.0469 m}$ is very small. So the assumption seems reasonable in these cases.

Finally consider the third level of the recursion. Let $\mathcal{N}^{\prime \prime}=\mathcal{N}\left(\frac{\mathcal{N}_{1}}{q^{\ell_{1}+\ell_{2}+\ell_{3}}}\right)\left(\frac{\mathcal{N}_{2}}{q^{\ell_{2}+\ell_{3}}}\right)^{2}$ be the number solutions to be split. Let $p_{3}=\frac{\mathcal{N}_{3}}{\# \mathcal{X}_{1 / 16+\alpha / 4+\beta / 2+\gamma, \alpha / 4+\beta / 2+\gamma}}$. For all values $\alpha, \beta, \gamma$ listed in Tables 2 and 3 we have $\max \left(p_{3}\right)=2^{-0.2123 m}$. Since $\mathcal{N}^{\prime \prime} \leq \mathcal{N} \leq 2^{0.2 m}$ (when $\mathcal{N}=1$, one chooses $q^{\ell_{1}+\ell_{2}+\ell_{3}} \approx \mathcal{N}_{1}$ and $q^{\ell_{2}+\ell_{3}} \approx \mathcal{N}_{2}$, while if $\overline{\mathcal{N}}>1$, one chooses $q^{\ell_{1}+\ell_{2}+\ell_{3}} \geq \mathcal{N}_{1}$ and $q^{\ell_{2}+\ell_{3}} \geq \mathcal{N}_{2}$ to reduce the time complexity), $p_{3} \mathcal{N}^{\prime \prime}=2^{-0.0123 m}$ is very small. Thus $\frac{1-\left(1-p_{3}\right)^{\mathcal{N}^{\prime \prime}}}{p_{3}} \mathcal{N}_{3} \approx \mathcal{N}^{\prime \prime} \mathcal{N}_{3}$, i.e., almost all the splittings of all the $\mathcal{N}^{\prime \prime}$ solutions at this stage are distinct.

Hence, when the number of solutions is $\leq 2^{0.2 m}$, Figure 1 seems to be an accurate view of the complexity of the HGJ and BCJ algorithms. When the exponential density is greater than $2^{0.2 m}$ then the results of Figure 1 are less rigorous, but they at least give some intuition for how the HGJ and BCJ algorithms behave. In any case, once the exponential density reaches around $2^{0.3 m}$ one would likely switch to the Minder-Sinclair variant of the CPW algorithm.

\section{Hermite Normal Form}

We now give the main idea of the paper. For simplicity, assume that $q$ is prime, $G=\mathbb{Z}_{q}^{n}$ and $n>1$. We also assume that the matrix $\mathbf{A}$ in the ISIS instance has rank equal to $n$, which will be true with very high probability when $m \gg n$.

We exploit the Hermite normal form. Given an $n \times m$ matrix $\mathbf{A}$ over $\mathbb{Z}_{q}$ with rank $n<m$ then, by permuting columns as necessary, we may assume that $\mathbf{A}=\left[\mathbf{A}_{1} \mid \mathbf{A}_{2}\right]$ where $\mathbf{A}_{1}$ is an invertible $n \times n$ matrix and $\mathbf{A}_{2}$ is an $n \times(m-n)$ matrix. Then there exists a matrix $\mathbf{U}=\mathbf{A}_{1}^{-1}$ such that

$$
\mathbf{U A}=\left[\mathbf{I}_{n} \mid \mathbf{A}^{\prime}\right]
$$


where $\mathbf{I}_{n}$ is the $n \times n$ identity matrix and $\mathbf{A}^{\prime}$ is the $n \times(m-n)$ matrix $\mathbf{U} \mathbf{A}_{2}$. The matrix $\left[\mathbf{I}_{n} \mid \mathbf{A}^{\prime}\right]$ is called the Hermite normal form (HNF) of $\mathbf{A}$ and it can be computed (together with $\mathbf{U}$ ) by various methods. We assume $q$ is prime and hence Gaussian elimination is sufficient to compute the HNF.

Writing $\mathbf{x}^{T}=\left(\mathbf{x}_{0}^{T} \mid \mathbf{x}_{1}^{T}\right)$ where $\mathbf{x}_{0}$ has length $n$ and $\mathbf{x}_{1}$ has length $m-n$ we have that

$$
\mathbf{s} \equiv \mathbf{A x} \quad(\bmod q) \quad \text { if and only if } \quad \mathbf{s}^{\prime}=\mathbf{U} \mathbf{s} \equiv \mathbf{A}^{\prime} \mathbf{x}_{1}+\mathbf{x}_{0} \quad(\bmod q)
$$

Hence, the Hermite normal form converts an ISIS instance to an approximate subset sum problem of the form $\mathbf{A}^{\prime} \mathbf{x} \approx \mathbf{s}^{\prime}$.

We now rename $\left(\mathbf{A}^{\prime}, \mathbf{s}^{\prime}, \mathbf{x}_{0}, \mathbf{x}_{1}\right)$ as $(\mathbf{A}, \mathbf{s}, \mathbf{e}, \mathbf{x})$ so the problem becomes $\mathbf{s}=\mathbf{A x}+\mathbf{e}$.

We will now apply the previous algorithms for the ISIS problem to this variant of the problem. This project was suggested in Section 6 of [15] to be an interesting problem (they called it the "approximate knapsack problem"). Our approach is to replace exact equality of elements in quotient groups $G / H=\mathbb{Z}_{q}^{\ell}$, in certain parts of the algorithm, by an approximate equality $\mathbf{y}_{1} \approx \mathbf{y}_{2}$. The definition of $\mathbf{y}_{1} \approx \mathbf{y}_{2}$ will be that $\mathbf{y}_{1}-\mathbf{y}_{2} \in \mathcal{E}$, where $\mathcal{E}$ is some neighborhood of $\mathbf{0}$. Different choices of $\mathcal{E}$ will lead to different tradeoffs of running time and success probability, and the exact choice depends somewhat on the algorithm under consideration.

\subsection{Approximate Merge Algorithm}

Our main tool is an algorithm to merge lists "approximately". We write $\mathbf{A x} \approx \mathbf{s}$ to mean $\mathbf{A x}+\mathbf{e}=\mathbf{s}$ for some $\mathbf{e} \in \mathcal{E}$ in some set $\mathcal{E}$ of permitted errors. We warn the reader that this symbol $\approx$ is not necessarily an equivalence relation (e.g., it is not necessarily symmetric or transitive).

We use similar notation to Sect. 2.3: $\mathcal{X} \subseteq \mathcal{B}^{m}$ is a set of vectors, letters $H_{i}$ denote suitably chosen subgroups of $G$ such that $\#\left(G / H_{i}\right)=q^{\ell_{i}}$. For any instance $\mathbf{s} \equiv \mathbf{A x}+\mathbf{e}$ we will split $\mathbf{x}=\mathbf{x}_{1}+\mathbf{x}_{2}$ for $\mathbf{x}_{1} \in \mathcal{X}_{1}$ and $\mathbf{x}_{2} \in \mathcal{X}_{2}$ and $\mathbf{e}=\mathbf{e}_{1}+\mathbf{e}_{2}$ for $\mathbf{e}_{1} \in \mathcal{E}^{(1)}$ and $\mathbf{e}_{2} \in \mathcal{E}^{(2)}$, where the sets $\mathcal{X}_{i}$ and $\mathcal{E}^{(i)}$ are appropriately chosen. Indeed, the error sets $\mathcal{E}^{(i)}$ are typically restricted to the quotient groups $G / H_{i}$, so that $\mathcal{E}^{(i)}=\mathcal{B}^{\ell_{i}}$. For example $\mathcal{E}^{(i)}=\{0,1\}^{\ell_{i}}$ for HGJ or $\{-1,0,1\}^{\ell_{i}}$ for BCJ.

Let $H^{\mathrm{b}}, H, H^{\sharp}$ be subgroups of $G$ that denote subgroups used in the CPW/HGJ/BCJ algorithms. We are merging, modulo $H$, partial solutions modulo $H^{b}$. For clarity, let us write $G / H^{b}=\mathbb{Z}_{q}^{\ell^{\prime}}$ and $G /\left(H \cap H^{b}\right)=\mathbb{Z}_{q}^{\ell+\ell^{\prime}}$. The input is a pair of lists $L_{1}$ and $L_{2}$ that are "partial solutions" modulo $H^{\mathrm{b}}$. In other words, they are lists of pairs $(\mathbf{x}, \mathbf{A x}$ $\left.\left(\bmod H^{b}\right)\right)$ such that if $\left(\mathbf{x}_{1}, \mathbf{A} \mathbf{x}_{1}\right) \in L_{1}$ and $\left(\mathbf{x}_{2}, \mathbf{A} \mathbf{x}_{2}\right) \in L_{2}$ then $\mathbf{A}\left(\mathbf{x}_{1}+\mathbf{x}_{2}\right)+\mathbf{e} \equiv \mathbf{s}$ (mod $H^{b}$ ) for some $\mathbf{e} \in \mathcal{E}^{b} \subset \mathcal{B}^{\ell^{\prime}}$. The goal is to output a set of solutions to the problem $\mathbf{A x}+\mathbf{e} \equiv \mathbf{s}\left(\bmod H \cap H^{b}\right)$ for $\mathbf{x} \in \mathcal{X}$ and, reusing notation, e $\in \mathcal{E} \subset \mathcal{B}^{\ell+\ell^{\prime}}$ (e.g., $\mathbf{e} \in \mathcal{E} \subset\{0,1\}^{\ell+\ell^{\prime}}$ for HGJ or $\mathbf{e} \in \mathcal{E} \subset\{-1,0,1\}^{\ell+\ell^{\prime}}$ for BCJ). We write $\mathcal{E}_{1}^{b}, \mathcal{E}_{2}^{b}$ for the error sets used in the lists $L_{1}, L_{2} ; \mathcal{E}$ for the error set for $G /\left(H^{\mathrm{b}} \cap H\right) ; \mathcal{E}^{\prime}$ for the error set corresponding to the elements of $G / H$. For future steps of the algorithm, the output includes information about $\mathbf{A x}\left(\bmod H^{\sharp}\right)$. The details are given in Algorithm 2.

The detection of values $\mathbf{u}^{\prime}$ in the sorted list such that $\mathbf{v} \approx \mathbf{u}^{\prime}$ (meaning $\mathbf{u}^{\prime}-\mathbf{v}=\mathbf{e}$ for some $\mathbf{e} \in \mathcal{E}^{\prime}$ ) can be done in several ways. One is to try all possible error vectors $\mathbf{e}$ and 


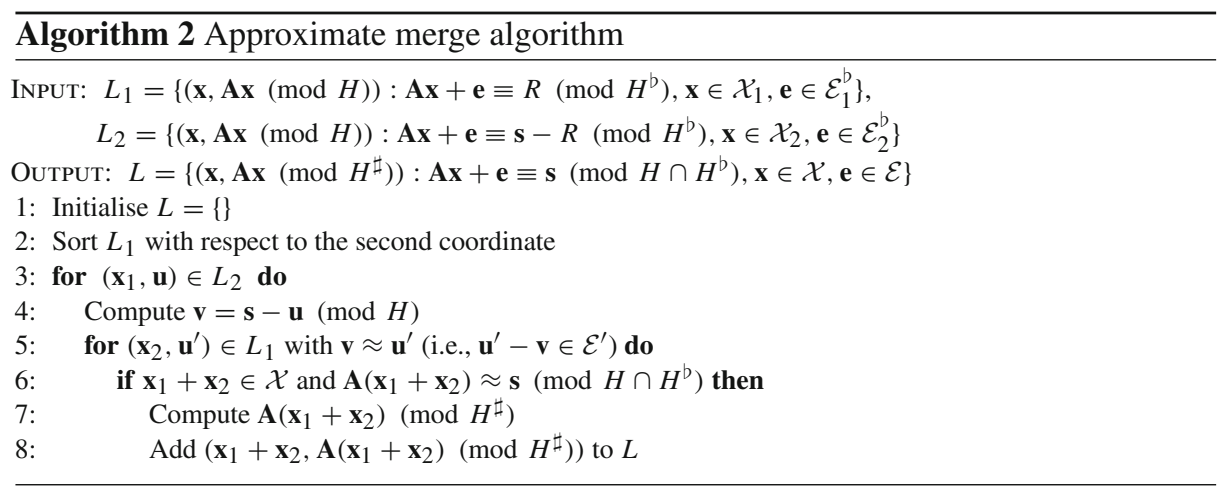

look up each candidate value $\mathbf{v}+\mathbf{e}$. Another is to "hash" using most significant bits; we give the details in Sect. 4.2 below. The running time of the algorithm depends on this choice. For each match we check the correctness for the whole quotient $G /\left(H \cap H^{b}\right)$.

Lemma 2. Let $G / H=\mathbb{Z}_{q}^{\ell}$ and let $\mathcal{E}^{\prime} \subseteq \mathbb{Z}_{q}^{\ell}$ be an error set for $G / H$. Suppose $\# L_{1}$ is exponentially large. Assuming Heuristic 1, Algorithm 2 performs

$$
\tilde{O}\left(\# L_{1} \cdot \log \left(\# L_{1}\right)+\# L_{2} \cdot\left\lceil\frac{\# L_{1} \cdot \# \mathcal{E}^{\prime}}{q^{\ell}}\right\rceil\right)
$$

arithmetic operations.

Here, by arithmetic operations, one can take operations in $\mathbb{Z}_{q}$. But when $\# L_{1}$ is exponentially large then all natural interpretations of "arithmetic operations" are equivalent by the $\tilde{O}$ notation.

Proof. Sorting $L_{1}$ takes $\tilde{O}\left(\# L_{1} \cdot \log \left(\# L_{1}\right)\right)$ operations on vectors in $G / H=\mathbb{Z}_{q}^{\ell}$.

For each pair $\left(\mathbf{x}_{1}, \mathbf{u}\right) \in L_{2}$ and each $\mathbf{e} \in \mathcal{E}^{\prime} \subseteq \mathbb{Z}_{q}^{\ell}$, Heuristic 1 implies that the expected number of "matches" $\left(\mathbf{x}_{2}, \mathbf{u}-\mathbf{e}\right)$ in $L_{1}$ is $\# L_{1} / q^{\ell}$. In the case where all values for $\mathbf{e} \in \mathcal{E}^{\prime}$ are chosen and then each candidate for $\mathbf{u}^{\prime}$ is looked up in the table, then the check in line 6 of the algorithm is performed

$$
\# L_{2} \cdot\left\lceil\frac{\# L_{1} \cdot \# \mathcal{E}^{\prime}}{q^{\ell}}\right\rceil .
$$

times. The most expensive arithmetic takes place in line 7 , which only executes when the checks in line 6 pass, but for simplicity (and since we are using $\tilde{O}$ ) we bound the running time as proportional to the above formula.

In the BCJ application in Sect. 4.3 we will always have $\# L_{1} \geq q^{\ell}$. Hence we can ignore the ceiling operation in the $\tilde{O}\left(\# L_{1} \# L_{2} /\left(q^{\ell} / \# \mathcal{E}^{\prime}\right)\right)$ term.

As previously, it is non-trivial to approximate the size of the output list $L$. Instead, this can be approximated by $\# \mathcal{X} \# \mathcal{E} / \#\left(G /\left(H \cap H^{b}\right)\right)$. 
Note that different choices for $\mathcal{E}, \mathcal{E}_{1}^{b}, \mathcal{E}_{2}^{b}, \mathcal{E}^{\prime}$ can lead to different organization in the algorithm. For example we may take the possible nonzero positions in $\mathcal{E}_{1}^{b}$ and $\mathcal{E}_{2}^{b}$ to be disjoint, then after executing line 5 of Algorithm 2 we always have $\mathbf{A}\left(\mathbf{x}_{1}+\mathbf{x}_{2}\right) \approx \mathbf{s}$ $\left(\bmod H^{b}\right)$, and so in line 6 of Algorithm 2 we only need to check $\mathbf{A}\left(\mathbf{x}_{1}+\mathbf{x}_{2}\right) \approx \mathbf{s}$ $(\bmod H)$; this is what we will do when adapting the CPW algorithm to this case.

\subsection{The CPW Algorithm}

Recall that our problem is (taking the HNF and then renaming $\left(\mathbf{A}^{\prime}, \mathbf{s}^{\prime}\right)$ as $(\mathbf{A}, \mathbf{s})$ and denoting $\left(\mathbf{x}_{1}, \mathbf{x}_{0}\right)$ as $\left.(\mathbf{x}, \mathbf{e})\right)$ : Given $\mathbf{A}, q, \mathbf{s}$ to solve $\mathbf{A x}+\mathbf{e}=\mathbf{s}$ in $G=\mathbb{Z}_{q}^{n}$, where $\mathbf{x} \in \mathcal{B}^{m-n}$ has length $m-n$ and $\mathbf{e} \in \mathcal{B}^{n}$ has length $n$. We assume the problem has high density, in the sense that there are many solutions $(\mathbf{x}, \mathbf{e}) \in \mathcal{B}^{m}$ to the system.

As we have seen, the CPW algorithm is most suitable for problems with very high density, since higher density means more lists can be used and so the running time is lower. Hence, it may seem that reducing $m$ to $m-n$ will be unhelpful for the CPW algorithm. However, we actually get a very useful tradeoff. In some sense, the high density is preserved in our transform while the actual computations are reduced due to the reduction in the size of $m$.

As noted, we define a (not necessarily symmetric or transitive) relation $\approx$ on vectors in $G=\mathbb{Z}_{q}^{n}$ as $\mathbf{v} \approx \mathbf{w}$ if and only if $\mathbf{v}-\mathbf{w} \in \mathcal{B}^{n}$. One can then organize a CPW-style algorithm: compute the lists $L_{j}^{(i)}$ as usual, but merge them using $\approx$. One needs to be a bit careful with the errors. We consider the following four-list CPW procedure as an example.

Example 1. Lists $L_{j}^{(0)}$ contain pairs $\left(\mathbf{x}, \mathbf{A x}\left(\bmod H_{1}\right)\right)\left(\operatorname{except} L_{4}^{(0)}\right.$ has pairs $(\mathbf{x}, \mathbf{A x}-\mathbf{s}$ $\left.\left.\left(\bmod H_{1}\right)\right)\right)$. Merging $L_{1}^{(0)}$ and $L_{2}^{(0)}$ gives a list $L_{1}^{(1)}$ of pairs $\left(\mathbf{x}_{1}+\mathbf{x}_{2}, \mathbf{A}\left(\mathbf{x}_{1}+\mathbf{x}_{2}\right)\right.$ $\left.\left(\bmod H_{2}\right)\right)$ for $\mathbf{x}_{1} \in L_{1}^{(0)}$ and $\mathbf{x}_{2} \in L_{2}^{(0)}$ such that $\mathbf{A}\left(\mathbf{x}_{1}+\mathbf{x}_{2}\right) \approx \mathbf{0}\left(\bmod \mathbf{H}_{1}\right)$, which means $\mathbf{A}\left(\mathbf{x}_{1}+\mathbf{x}_{2}\right) \equiv \mathbf{e}\left(\bmod H_{1}\right)$ for some $\mathbf{e} \in \mathcal{B}^{n / 3}$. Similarly, $L_{2}^{(1)}$ is a list of pairs $\left(\mathbf{x}_{3}+\mathbf{x}_{4}, \mathbf{A}\left(\mathbf{x}_{3}+\mathbf{x}_{4}\right)\left(\bmod H_{2}\right)\right)$ for $\mathbf{x}_{3} \in L_{3}^{(0)}$ and $\mathbf{x}_{4} \in L_{4}^{(0)}$ such that $\mathbf{A}\left(\mathbf{x}_{3}+\mathbf{x}_{4}\right)-\mathbf{s} \equiv \mathbf{e}^{\prime}$ $\left(\bmod H_{1}\right)$ for some $\mathbf{e}^{\prime} \in \mathcal{B}^{n / 3}$. The problem is that $\mathbf{e}+\mathbf{e}^{\prime}$ does not necessarily lie in $\mathcal{B}^{n / 3}$ and so the merge at the final stage will not necessarily lead to a solution to the problem.

There are several ways to avoid this issue. One would be to "weed out" these failed matches at the later stages. However, our approach is to constrain the relation $\approx$ further during the merge operations. Specifically (using the notation of the previous paragraph) we require the possible nonzero positions in $\mathbf{e}$ and $\mathbf{e}^{\prime}$ to be disjoint.

The details. Let $k=2^{t}$ be the number of lists. We define $u=(m-n) / k$ and let

$$
\mathcal{X}_{j}=\left\{\left(0, \ldots, 0, x_{(j-1) u+1}, \ldots, x_{j u}, 0, \ldots, 0\right) \in \mathcal{B}^{m-n}\right\}
$$

for $1 \leq j \leq k$. We choose integers $\ell_{i}>0$ such that $\ell_{1}+\ell_{2}+\cdots+\ell_{t}=n$ and then choose the subgroups $H_{i}$ so that $G / H_{i} \cong \mathbb{Z}_{q}^{\ell_{i}}$ for $1 \leq i \leq t$. So $H_{1}=$ 
$\left\{\left(0,0, \cdots, 0, g_{\ell_{1}+1}, \cdots, g_{n}\right) \in \mathbb{Z}_{q}^{n}\right\}, H_{2}=\left\{\left(g_{1}, \ldots, g_{\ell_{1}}, 0, \ldots, 0, g_{\ell_{1}+\ell_{2}+1}, \ldots, g_{n}\right)\right\}$ and so on.

Let $\gamma_{i}=\ell_{i} / 2^{t-i}=\ell_{i} /\left(k / 2^{i}\right)$ for $1 \leq i \leq t$. We assume in this section that the $\gamma_{i}$ are all integers; see Sect. 5 for an example of how to handle the case when $2^{t-i}$ does not divide $\ell_{i}$. In practice, the values of $\gamma_{i}$ can be computed from a integer program. For $1 \leq j \leq k / 2^{i}$ we define error sets $\mathcal{E}_{\gamma_{i}, j}^{(i)} \subseteq \mathcal{B}^{\ell_{i}}$ restricted to the quotient group $G / H_{i}$ and with $\gamma_{i}$ error positions as

$$
\mathcal{E}_{\gamma_{i}, j}^{(i)}=\left\{\left(0, \ldots, 0, e_{(j-1) \gamma_{i}+1}, \ldots, e_{j \gamma_{i}}, 0,0, \ldots, 0\right) \in \mathcal{B}^{\ell_{i}}\right\}
$$

Note that $\# \mathcal{E}_{\gamma_{i}, j}^{(i)}=(\# \mathcal{B})^{\gamma_{i}}$.

Level 0: $\quad$ Compute lists $L_{j}^{(0)}=\left\{\left(\mathbf{x}, \mathbf{A x}\left(\bmod H_{1}\right)\right) \in \mathcal{X}_{j} \times \mathbb{Z}_{q}^{\ell_{1}}\right\}$ for $1 \leq j \leq k-1$ and $L_{k}^{(0)}=\left\{\left(\mathbf{x}, \mathbf{A x}-\mathbf{s}\left(\bmod H_{1}\right)\right) \in \mathcal{X}_{k} \times \mathbb{Z}_{q}^{\ell_{1}}\right\}$. Note that $\# L_{j}^{(0)}=\# \mathcal{B}^{u}=\# \mathcal{B}^{(m-n) / k}$. The cost to compute the initial $k$ lists is $O\left(\# L_{j}^{(0)}\right)=O\left((\# \mathcal{B})^{(m-n) / k}\right)$ or, to be more precise, the cost is approximately $k \cdot \# L_{j}^{(0)} \cdot C$ bit operations, where $C$ is the number of bit operations to compute a sum of at most $u$ vectors in $\mathbb{Z}_{q}^{\ell_{1}}$, i.e., $C=(m-n) \log _{2}\left(q^{\ell_{1}}\right) / k$.

Level 1: We now merge the $k=2^{t}$ lists in pairs to get $k / 2=2^{t-1}$ lists. Recall that $\gamma_{1}=\ell_{1} /(k / 2)$. For $j=1,2, \cdots, k / 2$ the sets $\mathcal{E}_{\gamma_{1}, j}^{(1)} \in \mathcal{B}^{\ell_{1}}$ specify the positions that are allowed to contain errors. In other words, for $j=1,2, \cdots, k / 2-1$ we construct the new lists

$$
\begin{array}{r}
L_{j}^{(1)}=\left\{\left(\mathbf{x}_{1}+\mathbf{x}_{2}, \mathbf{A}\left(\mathbf{x}_{1}+\mathbf{x}_{2}\right) \quad\left(\bmod H_{2}\right)\right): \mathbf{x}_{1} \in L_{2 j-1}^{(0)}, \mathbf{x}_{2} \in L_{2 j}^{(0)},\right. \\
\left.\mathbf{A}\left(\mathbf{x}_{1}+\mathbf{x}_{2}\right) \quad\left(\bmod H_{1}\right) \in \mathcal{E}_{\gamma_{1}, j}^{(1)}\right\},
\end{array}
$$

and

$$
\begin{array}{r}
L_{k / 2}^{(1)}=\left\{\left(\mathbf{x}_{1}+\mathbf{x}_{2}, \mathbf{A}\left(\mathbf{x}_{1}+\mathbf{x}_{2}\right) \quad\left(\bmod H_{2}\right)\right): \mathbf{x}_{1} \in L_{k-1}^{(0)}, \mathbf{x}_{2} \in L_{k}^{(0)},\right. \\
\left.\mathbf{A}\left(\mathbf{x}_{1}+\mathbf{x}_{2}\right)-\mathbf{s} \quad\left(\bmod H_{1}\right) \in \mathcal{E}_{\gamma_{1}, k / 2}^{(1)}\right\} .
\end{array}
$$

The probability that two independent, uniformly random vectors in $\mathbb{Z}_{q}^{\ell_{1}}$ have $\operatorname{sum}$ in $\mathcal{E}_{\gamma_{1}, j}^{(1)}$ is $\# \mathcal{E}_{\gamma_{1}, j}^{(1)} / q^{\ell_{1}}=\# \mathcal{B}^{\gamma_{1}} / q^{\ell_{1}}$, and so the expected size of the lists $L_{j}^{(1)}$ is $\# L_{2 j-1}^{(0)} \# L_{2 j}^{(0)} \# \mathcal{B}^{\gamma_{1}} /$ $q^{\ell_{1}} \approx \# \mathcal{B}^{2(m-n) / k+\gamma_{1}} / q^{\ell_{1}}$.

Level $i \geq 2$ : The procedure continues in the same way. We are now merging $k / 2^{i-1}$ lists to get $k / 2^{i}$ lists. We do this by checking $\ell_{i}$ coordinates and so will allow errors for each merge in only $\gamma_{i}=\ell_{i} /\left(k / 2^{i}\right)$ positions. Hence, for $j=1,2, \cdots, k / 2^{i}-1$ we construct the new lists 


$$
\begin{array}{r}
L_{j}^{(i)}=\left\{\left(\mathbf{x}_{1}+\mathbf{x}_{2}, \mathbf{A}\left(\mathbf{x}_{1}+\mathbf{x}_{2}\right) \quad\left(\bmod H_{i+1}\right)\right): \mathbf{x}_{1} \in L_{2 j-1}^{(i-1)}, \mathbf{x}_{2} \in L_{2 j}^{(i-1)},\right. \\
\left.\mathbf{A}\left(\mathbf{x}_{1}+\mathbf{x}_{2}\right) \quad\left(\bmod H_{i}\right) \in \mathcal{E}_{\gamma_{i}, j}^{(i)}\right\},
\end{array}
$$

and

$$
\begin{array}{r}
L_{k / 2^{i}}^{(i)}=\left\{\left(\mathbf{x}_{1}+\mathbf{x}_{2}, \mathbf{A}\left(\mathbf{x}_{1}+\mathbf{x}_{2}\right) \quad\left(\bmod H_{i+1}\right)\right): \mathbf{x}_{1} \in L_{k / 2^{i-1}-1}^{(i-1)}, \mathbf{x}_{2} \in L_{k / 2^{i-1}}^{(i-1)},\right. \\
\left.\mathbf{A}\left(\mathbf{x}_{1}+\mathbf{x}_{2}\right)-\mathbf{s} \quad\left(\bmod H_{i}\right) \in \mathcal{E}_{\gamma_{i}, k / 2^{i}}^{(i)}\right\} .
\end{array}
$$

As before, the heuristic expected size of $L_{j}^{(i)}$ is $\# L_{2 j-1}^{(i-1)} \# L_{2 j}^{(i-1)} \# \mathcal{B}^{\gamma_{i}} / q^{\ell_{i}}$.

It remains to explain how to perform the merging of the lists using Algorithm 2. We are seeking a match on vectors in $\mathbb{Z}_{q}^{\ell_{i}}$ that are equal on all but $\gamma_{i}$ coordinates, and that are "close" on those $\gamma_{i}$ coordinates. The natural solution is to detect matches using the most significant bits of the coordinates (this approach was used in NTRU by HowgraveGraham, Silverman and Whyte [14]).

The Hash Function $F$. Precisely, let $v_{i}$ be a parameter (indicating the number of most significant bits being used). Represent $\mathbb{Z}_{q}$ as $\{0,1, \ldots, q-1\}$ and define a hash function $F: \mathbb{Z}_{q} \rightarrow \mathbb{Z}_{2^{v_{i}}}$ by $F(x)=\left\lfloor\frac{x}{q / 2^{v_{i}}}\right\rfloor$. We say that $a \in \mathbb{Z}$ is borderline if $F(a) \neq F(a+e)$ for a possible error value $e$. We can then extend $F$ to $\mathbb{Z}_{q}^{\gamma_{i}}$ (and to the whole of $\mathbb{Z}_{q}^{\ell_{i}}$ by taking the identity map on the other coordinates). We want to detect a match of the form $\mathbf{A} \mathbf{x}_{1}+\mathbf{A} \mathbf{x}_{2}+\mathbf{e}=\mathbf{0}$, which we will express as $-\mathbf{A} \mathbf{x}_{1}=\mathbf{A} \mathbf{x}_{2}+\mathbf{e}$. The idea is to compute $F\left(-\mathbf{A} \mathbf{x}_{1}\right)$ for all $\mathbf{x}_{1}$ in the first list and store these in a sorted list (or a hash table). For each value of $\mathbf{x}_{2}$ in the second list one computes all possible values for $F\left(\mathbf{A x}_{2}+\mathbf{e}\right)$ and checks which are in the first list.

Example 2. For example, consider $q=2^{3}=8$ and suppose we use a single most significant bit (so $v_{i}=1$ and $F: \mathbb{Z}_{q} \rightarrow\{0,1\}$ ). The borderline entries are 3 and 7 , which are both such that $F(a+1) \neq F(a)$. Suppose $-\mathbf{A x} \mathbf{x}_{1}=(7,2,4,5,6,4,0,7)^{T}$ and that we are only considering binary errors on the first 4 coordinates. Then we have $F\left(-\mathbf{A} \mathbf{x}_{1}\right)=(1,0,1,1,6,4,0,7)$. Suppose now $\mathbf{A} \mathbf{x}_{2}=(6,2,3,5,6,4,0,7)$. Then $F\left(\mathbf{A} \mathbf{x}_{2}\right)=(1,0,0,1,6,4,0,7)$. By looking at the borderline entries of $\mathbf{A} \mathbf{x}_{2}$ we know that we should also check $(1,0,1,1,6,4,0,7)$. There is no other value to check, since $F((6,2,3,5)+(1,1,0,1))=(1,0,0,1)$ and $F((6,2,3,5)+(1,1,1,1))=$ $(1,0,1,1)$ and so the only possible values for the first 4 entries of $F\left(\mathbf{A x}_{2}+\mathbf{e}\right)$ are $\{(1,0,0,1),(1,0,1,1)\}$.

To be precise we define $\operatorname{Flips}(\mathbf{v})=\left\{F(\mathbf{v}+\mathbf{e}): \mathbf{e} \in \mathcal{E}_{\gamma_{i}, j}^{(i)}\right\}$, where $i, j$ and $\gamma_{i}$ are clear in any given iteration of the algorithm. In other words, Flips( $(\mathbf{v})$ is the set of all patterns of most significant bits that would arise by adding valid errors to the corresponding coordinates of $\mathbf{v}$. It is important to realize that one does not need to loop over all $\mathbf{e} \in$ 
$\mathcal{E}_{\gamma_{i}, j}^{(i)}$ to compute Flips( $(\mathbf{v})$; instead one only needs to check entries of $\mathbf{v}$ that are on the borderline. As we will see, the set Flips(v) is usually quite small.

Suppose there are $r$ "borderline" integers in $\mathbb{Z}_{q}$ (meaning integers $0 \leq a<q$ such that $F(a) \neq F(a+e(\bmod q))$ for some $e \in \mathcal{B})$. Define $p_{\text {flip }}=r / q$ to be the probability that a randomly chosen element of $\mathbb{Z}_{q}$ has hash value that flips when adding a vector in $\mathcal{B}$. When $\mathcal{B}=\{0,1\}$ then the expected size of Flips $(a)$ is $((q-r) \cdot 1+r \cdot 2) / q=1+p_{\text {flip. }}$. Hence, for vectors $\mathbf{v} \in \mathbb{Z}_{q}^{\gamma_{i}}$ the expected size of Flips $(\mathbf{v})$ is $\left(p_{\text {flip }}+1\right)^{\gamma_{i}}$. More generally, given parameters $q, v_{i}$ and $\mathcal{B}^{\gamma_{i}}$, the value $p_{\text {flip }}$ and the expected value of \#Flips $(\mathbf{v})$ can be easily computed.

To summarize the "approximate merge" algorithm: First compute $F(\mathbf{v})$ for every $\mathbf{v}=-\mathbf{A} \mathbf{x}_{1}$ in the list $L_{2 j-1}^{(i-1)}$, and sort these values. Note that there may be multiple different values $\mathbf{x}_{1}$ in the list with the same hash value $F\left(-\mathbf{A} \mathbf{x}_{1}\right)$, and these should be stored in a list associated with that entry of the hash table or binary tree representing the sorted list. Then for every $\mathbf{v}=\mathbf{A} \mathbf{x}_{2}$ for $\mathbf{x}_{2}$ in the list $L_{2 j}^{(i-1)}$ we compute Flips(v) and search, for each $\mathbf{u} \in \operatorname{Flips}(\mathbf{v})$, for occurrences of $\mathbf{u}$ in the sorted list. Finally, for each match, we go through all values $\mathbf{x}_{1}$ in the first list with the given hash value $F\left(-\mathbf{A} \mathbf{x}_{1}\right)$ and, for each of them, check if it really is true that $\mathbf{A}\left(\mathbf{x}_{1}+\mathbf{x}_{2}\right)$ is in the correct error set (since a match of the hash values does not imply correctness). The number of possible hash values on vectors in $\mathbb{Z}_{q}^{\ell_{i}}$, with $\gamma_{i}$ positions reduced to the $v_{i}$ most significant bits, is around $2^{v_{i} \gamma_{i}} q^{\ell_{i}-\gamma_{i}}$. Hence, the average number of values in the list $L_{2 j-1}^{(i-1)}$ that take a given hash value is $\# L_{2 j-1}^{(i-1)} /\left(2^{v_{i} \gamma_{i}} q^{\ell_{i}-\gamma_{i}}\right)$. Finally, for all good matches we need to compute $\mathbf{A}\left(\mathbf{x}_{1}+\mathbf{x}_{2}\right)\left(\bmod H_{i+1}\right)$.

Success Probability. Now we give a heuristic analysis of the algorithm (again, we are considering the probability over the instances of the problem). The algorithm succeeds if $L_{1}^{(t)}$ is not empty, where $k=2^{t}$ is the number of lists used. We assume that the lists at the same level have the same size and denote by $L^{(i)}$ any of the lists at the $i$ th level of the algorithm. In practice, the list sizes at the same level may be different, and they can be obtained by solving an integer programming problem. The heuristic expected size of the lists on each level are

$$
\begin{aligned}
& \# L^{(0)}=(\# \mathcal{B})^{(m-n) / 2^{t}}, \\
& \# L^{(i)}=\frac{\# L^{(i-1)} \cdot \# L^{(i-1)} \cdot \# \mathcal{B}^{\gamma_{i}}}{q^{\ell_{i}}} \text { for } 1 \leq i \leq t .
\end{aligned}
$$

Following Minder and Sinclair we use integer programming to get optimal values for $\ell_{i}$. We write \#L $L^{(i)}=2^{b_{i}}$ where $b_{0}=(m-n) \log _{2}(\# \mathcal{B}) / 2^{t}$ and hence get $b_{i}=$ $2 b_{i-1}-\ell_{i} \log _{2}(q)+\gamma_{i} \log _{2}(\# \mathcal{B})$ where $\gamma_{i}=\ell_{i} / 2^{t-i}$. The time complexity is primarily proportional to $\max _{0 \leq i \leq t} \# L^{(i)}$ (it also depends to a lesser extent on $\gamma_{i}$ and the size of Flips). So to minimize the time one should choose $k=2^{t}$ to be as large as possible and then choose the $\ell_{i}$ to be a solution to the following integer program. 


$$
\begin{array}{ll}
\text { Minimize } & b_{\max }=\max _{0 \leq i \leq t} b_{i} . \\
\text { subject to } & 0 \leq b_{i} \leq b_{\max } \text { for } 0 \leq i \leq t, \\
& b_{0}=(m-n) \log _{2}(\# \mathcal{B}) / 2^{t}, \\
& b_{i}=2 b_{i-1}-\ell_{i} \log _{2}(q)+\ell_{i} \log _{2}(\# \mathcal{B}) / 2^{t-i}, \\
& \ell_{i} \geq 0 \text { for } 0 \leq i \leq t, \\
& \sum_{i=1}^{t} \ell_{i}=n .
\end{array}
$$

Complexity Analysis. We now fix a choice of $\ell_{1}, \ell_{2}, \cdots, \ell_{t}$ subject to the constraints mentioned above. Our aim is to give a precise formula for the running time of the CPW algorithm using our approximate merge algorithm. This will be used in Sect. 5 to analyze the running-time of the attacks on SWIFFT. Note that "approximate merge" is more complicated and takes more time than "basic merge", and so we have to investigate whether we have made a positive improvement overall.

Lemma 3. Let notation be as above. Let $C_{1}$ be the number of bit operations to compute $F$ for the $\gamma_{i}$ positions that allow errors. Let $C_{2}$ be the number of bit operations to compute Flips(v). Let $C_{3}$ be the number of bit operations to check that $\boldsymbol{x}_{1}+\boldsymbol{x}_{2} \in \mathcal{B}^{m-n}$ and that $\boldsymbol{A}\left(\boldsymbol{x}_{1}+\boldsymbol{x}_{2}\right)\left(\bmod H_{i}\right) \in \mathcal{E}_{\gamma_{i}, j}^{(i)}$ (we only need to check $\gamma_{i}$ positions of the error). Then at level $i$, Algorithm 2 requires on average

$$
\begin{aligned}
& \# L_{2 j-1}^{(i-1)}\left(C_{1}+\log _{2}\left(\# L_{2 j-1}^{(i-1)}\right) \log _{2}\left(q^{\ell_{i}}\right)\right) \\
& +\# L_{2 j}^{(i-1)}\left(C_{2}+\# \text { Flips }(v)\left[\log _{2}\left(\# L_{2 j-1}^{(i-1)}\right) \log _{2}\left(q^{\ell_{i}}\right)+C_{3} \frac{\# L_{2 j-1}^{(i-1)}}{2^{v_{i} \gamma_{i}} q^{\ell_{i}-\gamma_{i}}}\right]\right) \\
& +\# L_{j}^{(i)} \frac{(m-n)}{2^{t-i}} \log _{2}\left(q^{\ell_{i+1}}\right)
\end{aligned}
$$

bit operations.

Proof. Line 2 of Algorithm 2 requires computing $F\left(-\mathbf{A} \mathbf{x}_{1}\left(\bmod H_{i}\right)\right)$ for all elements in the list $L_{2 j-1}^{(i-1)}$ and then sorting. The total cost is bounded by $\# L_{2 j-1}^{(i-1)}\left(C_{1}+\right.$ $\left.\log _{2}\left(\# L_{2 j-1}^{(i-1)}\right) \log _{2}\left(q^{\ell_{i}}\right)\right)$ bit operations, which gives the component of equation (6).

Next, for each pair $\left(\mathbf{x}_{2}, \mathbf{v}\right) \in L_{2 j}^{(i-1)}$ one needs to compute Flips(v). For each element in Flips(v), one needs to look up the value in the sorted list. The notation \#Flips denotes the expected number of flips to be checked and it depends on the parameters $\gamma_{i}$ and $v_{i}$ in the approximate-merge. For each match one has, on average, $\# L_{2 j-1}^{(i-1)} /\left(2^{v_{i} \gamma_{i}} q^{\ell_{i}-\gamma_{i}}\right)$ different values from the first list with that hash value. Hence we need to handle around $\#$ Flips $(\mathbf{v}) \cdot \# L_{2 j}^{(i-1)} \# L_{2 j-1}^{(i-1)} /\left(2^{v_{i} \gamma_{i}} q^{\ell_{i}-\gamma_{i}}\right)$ values. For each of these we need to compute 
$\mathbf{A}\left(\mathbf{x}_{1}+\mathbf{x}_{2}\right)\left(\bmod H_{i}\right)$ and check that it is within the permitted error set. This leads to Eq. (7).

Finally, one needs to compute the values $\mathbf{A}\left(\mathbf{x}_{1}+\mathbf{x}_{2}\right)$ ( $\left.\bmod H_{i+1}\right)$ for the next iteration. This occurs only for the "good" values, of which there are by definition $\# L_{j}^{(i)}$. The computation of $\mathbf{A}\left(\mathbf{x}_{1}+\mathbf{x}_{2}\right)$ is adding ${ }^{6}$ at most $(m-n) / 2^{t-i}$ vectors of length $\ell_{i+1}$.

Note that Algorithm 2 is deterministic. By saying "on average", we are averaging over random inputs/problem instances.

Note also that Lemma 3 does not include the setup time of computing the initial lists. This should also be taken account when determining the total running time.

Larger values for $v_{i}$ (the number of bits in the output of $F$ ) increase $p_{\text {flip }}$ but reduce $\# L_{2 j-1}^{(i-1)} /\left(2^{v_{i} \gamma_{i}} q^{\ell_{i}-\gamma_{i}}\right)$. Note that $v_{i}$ does not effect the success probability, but only the running time. In practice, we first use a linear program to optimize the list sizes in terms of the parameters $\ell_{i}$ and $\gamma_{i}$. Once they are fixed, we choose $v_{i}$ to minimize the costs in equation (7) by balancing the terms \#Flips(v) and \#L $L_{2 j-1}^{(i-1)} /\left(2^{v_{i} \gamma_{i}} q^{\ell_{i}-\gamma_{i}}\right)$. The overall time to perform the "approximate merge" is proportional to $\# L_{2 j}^{(i-1)} \log _{2}\left(\# L_{2 j-1}^{(i-1)}\right)$ which is dominated by the list size in the procedure. Note if $\gamma_{i}=0$ and $C_{1}, C_{2}, C_{3}$ are 0 , we recover the basic merge algorithm and we have $v_{i}=\log _{2}(q)$, \#Flips $(\mathbf{v})=1$ and $\# L_{2 j-1}^{(i-1)} /\left(2^{v_{i} \gamma_{i}} q^{\ell_{i}-\gamma_{i}}\right)=\# L_{2 j-1}^{(i-1)} / q^{\ell_{i}}$.

Given a concrete instance of the problem, some of the constraints (such as that all the $\gamma_{i}$ are equal for the same level $i$ ) may not be satisfiable. We use an integer program to get a precise complexity statement together with Lemma 3. In Sect. 5 we use these ideas to obtain an algorithm for the SWIFFT hash function, and we demonstrate improved running-time estimates compared with the standard CPW algorithm.

\subsection{The HGJ and BCJ Algorithm}

Recall that our problem is: Given $\mathbf{A}, \mathbf{s}$ to solve $\mathbf{A x}+\mathbf{e}=\mathbf{s}$ in $G=\mathbb{Z}_{q}^{n}$, where $\mathbf{x}$ has length $m^{\prime}=m-n$ and $\mathbf{e}$ has length $n$. Here $\mathbf{A}$ is an $n \times m^{\prime}$ matrix, $\mathbf{x} \in\{0,1\}^{m^{\prime}}$ and $\mathbf{e} \in\{0,1\}^{n}$. We assume $\operatorname{wt}(\mathbf{x})=m^{\prime} / 2$ and $\operatorname{wt}(\mathbf{e})=n / 2$. Assume for simplicity ${ }^{7}$ that the density is approximately 1 (hence $2^{m^{\prime}+n} / q^{n} \approx 1$ ). Using Algorithm 2 we can get an improved version of the HGJ/BCJ algorithm. Since HGJ is a special case of BCJ, we discuss the general case.

We use the BCJ algorithm with 3 levels of recursion to find a solution $\mathbf{x} \in\{0,1\}^{m^{\prime}}$ of weight $m^{\prime} / 2$. Recall that $\mathcal{X}_{a, b}$ denotes vectors in $\{-1,0,1\}^{m^{\prime}}$ with $a m^{\prime}$ entries equal to 1 and $\mathrm{bm}^{\prime}$ entries equal to -1 . With this notation, our desired solution is an element of $\mathcal{X}_{1 / 2,0}$.

Following [4] we choose suitable parameters $\alpha, \beta, \gamma$ (see below). The first level of recursion splits $\mathbf{x}=\mathbf{x}_{1}+\mathbf{x}_{2}$ where $\mathbf{x}_{1}, \mathbf{x}_{2} \in \mathcal{X}_{1 / 4+\alpha, \alpha}$. The second level of recursion splits $\mathbf{x} \in \mathcal{X}_{1 / 4+\alpha, \alpha}$ into $\mathbf{x}_{1}+\mathbf{x}_{2}$ where $\mathbf{x}_{1}, \mathbf{x}_{2} \in \mathcal{X}_{1 / 8+\alpha / 2+\beta, \alpha / 2+\beta}$. The third level of recursion splits a vector into a sum of two vectors in the set $\mathcal{X}_{1 / 16+\alpha / 4+\beta / 2+\gamma, \alpha / 4+\beta / 2+\gamma}$.

\footnotetext{
${ }^{6}$ This is for $\mathcal{B}=\{0,1\}$; it is adding or subtracting when $\mathcal{B}=\{-1,0,1\}$.

${ }^{7}$ We do not analyze this algorithm in the case of density $>1$, but it is clear that similar improvements can be obtained as in Sect. 3. As in Sect. 3 it is not possible to give a general formula for the running time.
} 
We also use the BCJ idea to split the errors. This is a little more complex to describe as the error sets are in $\{-1,0,1\}^{n^{\prime}}$ for varying values of $n^{\prime}<n$. The notation $\mathcal{E}_{a, b}$ will mean a set of vectors in $\{-1,0,1\}^{n^{\prime}}$ with $a n^{\prime}$ entries equal to 1 and $b n^{\prime}$ entries equal to -1 . We assume $\mathbf{e} \in \mathcal{E}_{1 / 2,0}$ initially $\left(n^{\prime}=n\right.$ ). We will fix parameters $\alpha^{\prime}, \beta^{\prime}, \gamma^{\prime}$ (to be specified below).

Let $H_{1}, H_{2}, H_{3}$ be subgroups of $G$, of the usual form, such that $\#\left(G / H_{i}\right)=q^{\ell_{i}}$. For $1 \leq i \leq 3$ we define $\mathcal{E}_{a, b}^{(i \rightarrow 3)} \subseteq\{-1,0,1\}^{n^{\prime}}$ where $n^{\prime}=\ell_{i}+\cdots+\ell_{3}$ to be the set of vectors with $a n^{\prime}$ entries equal to 1 and $b n^{\prime}$ entries equal to -1 . These are considered as subsets of the quotient groups $G /\left(H_{i} \cap \cdots \cap H_{3}\right)$. We denote $\mathcal{E}_{a, b}^{(3 \rightarrow 3)}$ by $\mathcal{E}_{a, b}^{(3)}$.

Algorithm. The first level of recursion chooses a random value $R_{1} \in \mathbb{Z}_{q}^{\ell_{1}+\ell_{2}+\ell_{3}}=$ $G /\left(H_{1} \cap H_{2} \cap H_{3}\right)$ and computes two lists $L_{1}^{(1)}=\left\{(\mathbf{x}, \mathbf{A x}): \mathbf{x} \in \mathcal{X}_{1 / 4+\alpha, \alpha}, \mathbf{A x}+\mathbf{e} \equiv R_{1}\right.$ $\left(\bmod H_{1} \cap H_{2} \cap H_{3}\right)$ for some $\left.\mathbf{e} \in \mathcal{E}_{1 / 4+\alpha^{\prime}, \alpha^{\prime}}^{(1 \rightarrow 3)}\right\}$ and $L_{2}^{(1)}$ which is the same except $\mathbf{A x}+\mathbf{e} \equiv \mathbf{s}-R_{1}\left(\bmod H_{1} \cap H_{2} \cap H_{3}\right)$.

The second level of recursion computes $L_{1}^{(1)}$ and $L_{2}^{(1)}$ by splitting into further lists. We split $\mathbf{x} \in \mathcal{X}_{1 / 4+\alpha, \alpha}$ into $\mathbf{x}_{1}+\mathbf{x}_{2}$ where $\mathbf{x}_{1}, \mathbf{x}_{2} \in \mathcal{X}_{1 / 8+\alpha / 2+\beta, \alpha / 2+\beta}$ and split error vectors into $\mathcal{E}_{1 / 8+\alpha^{\prime} / 2+\beta^{\prime}, \alpha^{\prime} / 2+\beta^{\prime}}^{(2 \rightarrow 3)}$. For example, choosing a random value $R_{2}, L_{1}^{(1)}$ is split into $L_{1}^{(2)}=\left\{(\mathbf{x}, \mathbf{A x}): \mathbf{x} \in \mathcal{X}_{1 / 8+\alpha / 2+\beta, \alpha / 2+\beta}, \mathbf{A x}+\mathbf{e} \equiv R_{2}\left(\bmod H_{2} \cap H_{3}\right)\right.$, for some $\mathbf{e} \in \mathcal{E}_{1 / 8+\alpha^{\prime} / 2+\beta^{\prime}, \alpha^{\prime} / 2+\beta^{\prime}}^{(2 \rightarrow 3)}$ and $L_{2}^{(2)}$ is similar except the congruence is $\mathbf{A x}+\mathbf{e} \equiv R_{1}-R_{2}$ $\left(\bmod \mathrm{H}_{2} \cap \mathrm{H}_{3}\right)$.

The final level of recursion computes each list $L_{j}^{(2)}$ by splitting $\mathbf{x} \in \mathcal{X}_{1 / 8+\alpha / 2+\beta, \alpha / 2+\beta}$ into a sum $\mathcal{X}_{1 / 16+\alpha / 4+\beta / 2+\gamma, \alpha / 4+\beta / 2+\gamma}$ and splitting the error $\mathbf{e}\left(\bmod H_{3}\right) \in$ $\mathcal{E}_{1 / 8+\alpha^{\prime} / 2+\beta^{\prime}, \alpha^{\prime} / 2+\beta^{\prime}}^{(2 \rightarrow 3)}$ into a sum from $\mathcal{E}_{1 / 16+\alpha^{\prime} / 4+\beta^{\prime} / 2+\gamma^{\prime}, \alpha^{\prime} / 4+\beta^{\prime} / 2+\gamma^{\prime}}^{(3)}$ The lists at the final level are computed using the Shroeppel-Shamir algorithm.

Success Probability. Suppose there is a unique solution $(\mathbf{x}, \mathbf{e}) \in\{0,1\}^{m^{\prime}+n}$ to the $G$ ISIS instance, where $\mathbf{x}$ has weight $m^{\prime} / 2$ and $\mathbf{e}$ has weight $n / 2$. Consider the first step of the recursion. For the whole algorithm to succeed, it is necessary that there is a splitting $\mathbf{x}=\mathbf{x}_{1}+\mathbf{x}_{2}$ of the solution together with a splitting $\mathbf{e} \equiv \mathbf{e}_{1}+\mathbf{e}_{2}\left(\bmod H_{1} \cap H_{2} \cap H_{3}\right)$, so that for a randomly chosen $R_{1} \in G /\left(H_{1} \cap H_{2} \cap H_{3}\right), \mathbf{A} \mathbf{x}_{1}+\mathbf{e}_{1} \equiv R_{1}\left(\bmod H_{1} \cap H_{2} \cap H_{3}\right)$ and $\mathbf{A} \mathbf{x}_{2}+\mathbf{e}_{2} \equiv \mathbf{s}-R_{1}\left(\bmod H_{1} \cap H_{2} \cap H_{3}\right)$. The number of ways to split $\mathbf{x}$ (with length $m^{\prime}$ ) on the first level of recursion is

$$
\mathcal{N}_{1}=\left(\begin{array}{l}
m^{\prime} / 2 \\
m^{\prime} / 4
\end{array}\right)\left(\begin{array}{c}
m^{\prime} / 2 \\
\alpha m^{\prime}, \alpha m^{\prime},(1 / 2-2 \alpha) m^{\prime}
\end{array}\right)
$$

where the notation $\left(\begin{array}{c}N \\ a, b,(N-a-b)\end{array}\right)=\left(\begin{array}{c}N \\ a, b\end{array}\right)=\left(\begin{array}{c}N \\ a\end{array}\right)\left(\begin{array}{c}N-a \\ b\end{array}\right)$ denotes the usual multinomial coefficient. The number of ways to splite $\left(\bmod H_{1} \cap H_{2} \cap H_{3}\right)$ (with length $\left.\ell_{1}+\ell_{2}+\ell_{3}\right)$ on the first level of recursion is

$$
\mathcal{N}_{1}^{\prime}=\left(\begin{array}{c}
\left(\ell_{1}+\ell_{2}+\ell_{3}\right) / 2 \\
\left(\ell_{1}+\ell_{2}+\ell_{3}\right) / 4
\end{array}\right)\left(\begin{array}{c}
\left(\ell_{1}+\ell_{2}+\ell_{3}\right) / 2 \\
\alpha^{\prime}\left(\ell_{1}+\ell_{2}+\ell_{3}\right), \alpha^{\prime}\left(\ell_{1}+\ell_{2}+\ell_{3}\right)
\end{array}\right) .
$$


For randomly chosen $R_{1} \in G /\left(H_{1} \cap H_{2} \cap H_{3}\right)$, we expect there to be a valid splitting if $\mathcal{N}_{1} \mathcal{N}_{1}^{\prime} \geq q^{\ell_{1}+\ell_{2}+\ell_{3}}$. Indeed, the expected number of valid splittings should be roughly $\mathcal{N}_{1} \mathcal{N}_{1}^{\prime} / q^{\ell_{1}+\ell_{2}+\ell_{3}}$. Hence, we choose $\mathcal{N}_{1} \mathcal{N}_{1}^{\prime} \approx q^{\ell_{1}+\ell_{2}+\ell_{3}}$ to make sure a valid splitting pair exists at this stage with significant probability.

For the second stage we assume that we already made a good choice in the first stage, and indeed that we have $\mathcal{N}_{1} \mathcal{N}_{1}^{\prime} / q^{\ell_{1}+\ell_{2}+\ell_{3}}$ possible values for $\left(\mathbf{x}_{1}, \mathbf{e}_{1}\right)$ where $\mathbf{x}_{1} \in \mathcal{X}_{1 / 4+\alpha, \alpha}, \mathbf{e}_{1} \in \mathcal{E}_{1 / 4+\alpha^{\prime}, \alpha^{\prime}}^{(1 \rightarrow 3)}$. The number of ways to further split $\mathbf{x}_{1}$ is

$$
\mathcal{N}_{2}=\left(\begin{array}{c}
(1 / 4+\alpha) m^{\prime} \\
(1 / 8+\alpha / 2) m^{\prime}
\end{array}\right)\left(\begin{array}{c}
\alpha m^{\prime} \\
\alpha m^{\prime} / 2
\end{array}\right)\left(\begin{array}{c}
(3 / 4-2 \alpha) m^{\prime} \\
\beta m^{\prime}, \beta m^{\prime},(3 / 4-2 \alpha-2 \beta) m^{\prime}
\end{array}\right)
$$

The number of ways to further split $\mathbf{e}_{1}\left(\bmod H_{2} \cap H_{3}\right)$ is

$$
\begin{aligned}
\mathcal{N}_{2}^{\prime}= & \left(\begin{array}{c}
\left(1 / 4+\alpha^{\prime}\right)\left(\ell_{2}+\ell_{3}\right) \\
\left(1 / 8+\alpha^{\prime} / 2\right)\left(\ell_{2}+\ell_{3}\right)
\end{array}\right)\left(\begin{array}{c}
\alpha^{\prime}\left(\ell_{2}+\ell_{3}\right) \\
\alpha^{\prime}\left(\ell_{2}+\ell_{3}\right) / 2
\end{array}\right) \\
& \left(\begin{array}{c}
\left(3 / 4-2 \alpha^{\prime}\right)\left(\ell_{2}+\ell_{3}\right) \\
\beta^{\prime}\left(\ell_{2}+\ell_{3}\right), \beta^{\prime}\left(\ell_{2}+\ell_{3}\right),\left(3 / 4-2 \alpha^{\prime}-2 \beta^{\prime}\right)\left(\ell_{2}+\ell_{3}\right)
\end{array}\right) .
\end{aligned}
$$

The expected number of valid splittings at this stage should be roughly

$$
\left(\mathcal{N}_{1} \mathcal{N}_{1}^{\prime} / q^{\ell_{1}+\ell_{2}+\ell_{3}}\right)\left(\mathcal{N}_{2} \mathcal{N}_{2}^{\prime} / q^{\ell_{2}+\ell_{3}}\right)^{2}
$$

since at this stage we need to split both $\mathbf{x}_{1}$ and $\mathbf{x}_{2}$.

For a randomly chosen $R_{2} \in G /\left(H_{2} \cap H_{3}\right)$, there is a good chance that a valid splitting exists if

$$
\left(\mathcal{N}_{1} \mathcal{N}_{1}^{\prime} / q^{\ell_{1}+\ell_{2}+\ell_{3}}\right)\left(\mathcal{N}_{2} \mathcal{N}_{2}^{\prime} / q^{\ell_{2}+\ell_{3}}\right)^{2} \geq 1
$$

Hence, since we already choose $\mathcal{N}_{1} \mathcal{N}_{1}^{\prime} \approx q^{\ell_{1}+\ell_{2}+\ell_{3}}$, we now impose the condition $\mathcal{N}_{2} \mathcal{N}_{2}^{\prime} \approx q^{\ell_{2}+\ell_{3}}$.

In the final stage (again assuming good splittings in the entire second stage), the number of ways to split elements in $\mathcal{X}_{1 / 8+\alpha / 2+\beta, \alpha / 2+\beta}$ is

$$
\mathcal{N}_{3}=\left(\begin{array}{c}
(1 / 8+\alpha / 2+\beta) m^{\prime} \\
(1 / 16+\alpha / 4+\beta / 2) m^{\prime}
\end{array}\right)\left(\begin{array}{c}
(\beta+\alpha / 2) m^{\prime} \\
(\beta / 2+\alpha / 4) m^{\prime}
\end{array}\right)\left(\begin{array}{c}
(7 / 8-\alpha-2 \beta) m^{\prime} \\
\gamma m^{\prime}, \gamma m^{\prime},(7 / 8-\alpha-2 \beta-2 \gamma) m^{\prime}
\end{array}\right) .
$$

The number of ways to split elements in $\mathcal{E}_{1 / 8+\alpha^{\prime} / 2+\beta^{\prime}, \alpha^{\prime} / 2+\beta^{\prime}}^{(3)}$ is

$$
\begin{aligned}
\mathcal{N}_{3}^{\prime}= & \left(\begin{array}{c}
\left(1 / 8+\alpha^{\prime} / 2+\beta^{\prime}\right) \ell_{3} \\
\left(1 / 16+\alpha^{\prime} / 4+\beta^{\prime} / 2\right) \ell_{3}
\end{array}\right)\left(\begin{array}{c}
\left(\beta^{\prime}+\alpha^{\prime} / 2\right) \ell_{3} \\
\left(\beta^{\prime} / 2+\alpha^{\prime} / 4\right) \ell_{3}
\end{array}\right) \\
& \left(\begin{array}{c}
\left(7 / 8-\alpha^{\prime}-2 \beta^{\prime}\right) \ell_{3} \\
\gamma^{\prime} \ell_{3}, \gamma^{\prime} \ell_{3},\left(7 / 8-\alpha^{\prime}-2 \beta^{\prime}-2 \gamma^{\prime}\right) \ell_{3}
\end{array}\right) .
\end{aligned}
$$


The expected number of valid splittings is

$$
\left(\mathcal{N}_{1} \mathcal{N}_{1}^{\prime} / q^{\ell_{1}+\ell_{2}+\ell_{3}}\right)\left(\mathcal{N}_{2} \mathcal{N}_{2}^{\prime} / q^{\ell_{2}+\ell_{3}}\right)^{2}\left(\mathcal{N}_{3} \mathcal{N}_{3}^{\prime} / q^{\ell_{3}}\right)^{4}
$$

which we require to be $\geq 1$. Hence, we add the additional constraint $\mathcal{N}_{3} \mathcal{N}_{3}^{\prime} \approx q^{\ell_{3}}$. Thus, choosing $\# G / H_{3}$ close to $\mathcal{N}_{3} \mathcal{N}_{3}^{\prime}, \# G /\left(H_{2} \cap H_{3}\right)$ close to $\mathcal{N}_{2} \mathcal{N}_{2}^{\prime}$ and $\# G /\left(H_{1} \cap\right.$ $H_{2} \cap H_{3}$ ) close to $\mathcal{N}_{1} \mathcal{N}_{1}^{\prime}$ then the heuristic success probability of the algorithm should be noticeable.

Parameters. Suitable parameters need to be chosen to optimize the running time and have good success probability. As in previous sections, we use the estimate $\# \mathcal{X}_{a, b} \approx$ $2^{H(a, b) \cdot m^{\prime}}$ where

$$
H(x, y)=-x \log _{2}(x)-y \log _{2}(y)-(1-x-y) \log _{2}(1-x-y)
$$

and similarly for $\mathcal{E}_{a, b}$. We also use

$$
h(x)=-x \log _{2}(x)-(1-x) \log _{2}(1-x)
$$

to estimate the binomial. Then the logarithm of the number of decompositions for each level is

$$
\begin{aligned}
& \log _{2}\left(\mathcal{N}_{1}\right) \approx \frac{m^{\prime}}{2}(h(1 / 2)+H(2 \alpha, 2 \alpha)) \approx m^{\prime} \frac{1+H(2 \alpha, 2 \alpha)}{2}, \\
& \log _{2}\left(\mathcal{N}_{2}\right) \approx m^{\prime} \cdot \frac{1+8 \alpha+(3-8 \alpha) \cdot H\left(\frac{\beta}{3 / 4-2 \alpha}, \frac{\beta}{3 / 4-2 \alpha}\right)}{4}, \\
& \log _{2}\left(\mathcal{N}_{3}\right) \approx m^{\prime} \cdot \frac{1+8 \alpha+16 \beta+(7-8 \alpha-16 \beta) \cdot H\left(\frac{\gamma}{7 / 8-\alpha-2 \beta}, \frac{\gamma}{7 / 8-\alpha-2 \beta}\right)}{8} .
\end{aligned}
$$

The (logarithmic) number of decompositions for the error vector is similar.

$$
\begin{aligned}
\log _{2}\left(\mathcal{N}_{1}^{\prime}\right) & \approx\left(\ell_{1}+\ell_{2}+\ell_{3}\right) \cdot \frac{1+H\left(2 \alpha^{\prime}, 2 \alpha^{\prime}\right)}{2} \\
\log _{2}\left(\mathcal{N}_{2}^{\prime}\right) & \approx\left(\ell_{2}+\ell_{3}\right) \cdot \frac{1+8 \alpha^{\prime}+\left(3-8 \alpha^{\prime}\right) \cdot H\left(\frac{\beta^{\prime}}{3 / 4-2 \alpha^{\prime}}, \frac{\beta^{\prime}}{3 / 4-2 \alpha^{\prime}}\right)}{4} \\
\log _{2}\left(\mathcal{N}_{3}^{\prime}\right) & \approx \ell_{3} \cdot \frac{1+8 \alpha^{\prime}+16 \beta^{\prime}+\left(7-8 \alpha^{\prime}-16 \beta^{\prime}\right) \cdot H\left(\frac{\gamma^{\prime}}{7 / 8-\alpha^{\prime}-2 \beta^{\prime}}, \frac{\gamma^{\prime}}{7 / 8-\alpha^{\prime}-2 \beta^{\prime}}\right)}{8} .
\end{aligned}
$$

Assuming that $2^{m^{\prime}+n} \approx q^{n}$, we have $n \approx \frac{m^{\prime}}{\log _{2} q-1}$, and we choose the following parameters (these are the result of an optimization problem to minimize the running time of the algorithm). 


$$
\begin{aligned}
& \alpha=0.0267, \beta=0.0168, \gamma=0.0029 \\
& \alpha^{\prime}=0.0279, \beta^{\prime}=0.0027, \gamma^{\prime}=0.0027 \\
& q^{\ell_{1}}=2^{0.2673 m^{\prime}} 2^{0.4558 n}, q^{\ell_{2}}=2^{0.2904 m^{\prime}} 2^{0.1388 n} \text { and } q^{\ell_{3}}=2^{0.2408 m^{\prime}} 2^{0.0507 n} .
\end{aligned}
$$

For such parameters, we can estimate

$$
\mathcal{N}_{1} \approx 2^{0.7985 m^{\prime}}, \mathcal{N}_{2}=2^{0.5312 m^{\prime}}, \text { and } \mathcal{N}_{3}=2^{0.2408 m^{\prime}}
$$

and

$$
\mathcal{N}_{1}^{\prime}=2^{0.8082\left(\ell_{1}+\ell_{2}+\ell_{3}\right)}, \mathcal{N}_{2}^{\prime}=2^{0.3568\left(\ell_{2}+\ell_{3}\right)} \text { and } \mathcal{N}_{3}^{\prime}=2^{0.2106 \ell_{3}} \text {. }
$$

We consider the success probability. The number of splittings for the first level is

$$
\mathcal{N}_{1} \mathcal{N}_{1}^{\prime} / q^{\ell_{1}+\ell_{2}+\ell_{3}}
$$

With the above parameters, then the logarithm (base 2) of this value is approximately

$$
\begin{aligned}
& 0.7985 m^{\prime}-\frac{0.7985 m^{\prime 2}}{m^{\prime}+n}-\frac{0.7985 m^{\prime} n}{m^{\prime}+n}-\frac{0.1238 n^{2}}{m^{\prime}+n} \\
& \approx-\frac{0.1238 n^{2}}{m^{\prime}+n}
\end{aligned}
$$

Similarly, the logarithm of the number of valid splittings for the second stage is

$$
\log _{2}\left(\left(\mathcal{N}_{1} \mathcal{N}_{1}^{\prime} / q^{\ell_{1}+\ell_{2}+\ell_{3}}\right)\left(\mathcal{N}_{2} \mathcal{N}_{2}^{\prime} / q^{\ell_{2}+\ell_{3}}\right)^{2}\right) \approx-\frac{0.3676 n^{2}}{m^{\prime}+n}
$$

Finally, the logarithm of the number of valid splittings for the last stage is

$$
\log _{2}\left(\left(\mathcal{N}_{1} \mathcal{N}_{1}^{\prime} / q^{\ell_{1}+\ell_{2}+\ell_{3}}\right)\left(\mathcal{N}_{2} \mathcal{N}_{2}^{\prime} / q^{\ell_{2}+\ell_{3}}\right)^{2}\left(\mathcal{N}_{3} \mathcal{N}_{3}^{\prime} / q^{\ell_{3}}\right)^{4}\right) \approx-\frac{0.5278 n^{2}}{m^{\prime}+n} .
$$

For example, taking $m=1024, n=64$ and $m^{\prime}=m-n$ gives expected number of splittings at each level to be $0.7,0.36$ and 0.23 respectively, giving a total success probability of approximately 0.06 . So we expect to need to repeat the algorithm around 16 times. For fixed $n$ and letting $m^{\prime} \rightarrow \infty$ then the success probability tends to 1 .

Heuristic Running Time. We now consider the running time for one execution of the algorithm. (We do not consider the number of repetitions required due to the success probability here.)

Theorem 2. Let $\boldsymbol{s}=\boldsymbol{A} \boldsymbol{x}+\boldsymbol{e}$ in $\mathbb{Z}_{q}^{n}$ where $\boldsymbol{x} \in\{0,1\}^{m^{\prime}}$ has weight $m^{\prime} / 2$, and $\boldsymbol{e} \in\{0,1\}^{n}$ has weight $n / 2$. Suppose the density is approximately 1 , so that $2^{m}=2^{m^{\prime}+n} \approx q^{n}$. With notation as above, and assuming heuristics about the regularity of $\boldsymbol{A x}+\boldsymbol{e}$, the approximate BCJ algorithm runs in $\tilde{O}\left(2^{0.2912 m^{\prime}+0.1899 n}\right)$ time. 
Proof. We run the algorithm as described using the parameters specified above.

First level. In the first level of the recursion, the expected size of each list is given by

$$
\begin{aligned}
& \log _{2}\left(\# L^{(1)}\right)=\log _{2}\left(\# \mathcal{X}_{1 / 4+\alpha, \alpha} \# \mathcal{E}_{1 / 4+\alpha^{\prime}, \alpha^{\prime}}^{(1 \rightarrow 3)} / q^{\ell_{1}+\ell_{2}+\ell_{3}}\right) \\
& \approx m^{\prime} H(1 / 4+\alpha, \alpha)+H\left(1 / 4+\alpha^{\prime}, \alpha^{\prime}\right)\left(\ell_{1}+\ell_{2}+\ell_{3}\right)-\left(\ell_{1}+\ell_{2}+\ell_{3}\right) \log _{2}(q) .
\end{aligned}
$$

For our parameters we have the relation

$$
q^{\ell_{1}+\ell_{2}+\ell_{3}}=2^{0.7985 m^{\prime}} 2^{0.6453 n} .
$$

Hence the logarithmic size is

$$
\begin{aligned}
\log _{2}\left(\# L^{(1)}\right) & =0.2173 m^{\prime}+0.8169 \frac{m^{\prime} n}{m^{\prime}+n}-0.6453 n+0.6601 \frac{n^{2}}{m^{\prime}+n} \\
& \leq 0.2173 m^{\prime}+0.1716 n
\end{aligned}
$$

Using the approximate merge (Algorithm 2) this requires time

$$
T_{1}=\tilde{O}\left(\# L^{(1)} \# L^{(1)} 2^{n-\ell_{1}-\ell_{2}-\ell_{3}} / q^{n-\ell_{1}-\ell_{2}-\ell_{3}}\right)
$$

One can compute a precise formula for $\log _{2}\left(T_{1}\right)$ by evaluating Eq. (9) with our parameters. We used a computer script to keep track of all the quantities. One can show that $\log _{2}\left(T_{1}\right) \leq 0.2331 m^{\prime}+0.1899 n$.

Second level. Similarly, in the second level of recursion the size of the lists is approximately

$$
\begin{aligned}
\log _{2}\left(\# L^{(2)}\right)= & \log _{2}\left(\# \mathcal{X}_{1 / 8+\alpha / 2+\beta, \alpha / 2+\beta} \# \mathcal{E}_{1 / 8+\alpha^{\prime} / 2+\beta^{\prime}, \alpha^{\prime} / 2+\beta^{\prime}}^{(2 \rightarrow 3)} / q^{\ell_{2}+\ell_{3}}\right) \\
\approx & m^{\prime} H(1 / 8+\alpha / 2+\beta, \alpha / 2+\beta) \\
& +H\left(1 / 8+\alpha^{\prime} / 2+\beta^{\prime}, \alpha^{\prime} / 2+\beta^{\prime}\right)\left(\ell_{2}+\ell_{3}\right)-\left(\ell_{2}+\ell_{3}\right) \log _{2}(q) .
\end{aligned}
$$

For our parameters we have the relation

$$
q^{\ell_{2}+\ell_{3}}=2^{0.5312 m^{\prime}} 2^{0.1895 n}
$$

Hence the logarithmic size is

$$
\begin{aligned}
\log _{2}\left(\# L^{(2)}\right) & =0.2791 m^{\prime}+\frac{0.3756 m^{\prime} n}{m^{\prime}+n}-0.1895 n+\frac{0.1340 n^{2}}{m^{\prime}+n} \\
& \leq 0.2791 m^{\prime}+0.1861 n
\end{aligned}
$$

Using the approximate merge (Algorithm 2), the cost to merge is

$$
T_{2}=\tilde{O}\left(\# L^{(2)} \# L^{(2)} \# \mathcal{E}_{1 / 4+\alpha^{\prime}, \alpha^{\prime}}^{(1)} / q^{\ell_{1}}\right) .
$$


With the above parameters

$$
\begin{aligned}
\log _{2}\left(T_{2}\right) & \approx 0.2908 m^{\prime}+\frac{1.0246 m^{\prime} n}{m^{\prime}+n}-0.8348 n+\frac{0.7342 n^{2}}{m^{\prime}+n} \\
& \leq 0.2908 m^{\prime}+0.1898 n
\end{aligned}
$$

Third level. The last level of recursion computes lists of expected size

$$
\begin{aligned}
\log _{2}\left(\# L^{(3)}\right)= & \log _{2}\left(\# \mathcal{X}_{1 / 16+\alpha / 4+\beta / 2+\gamma, \alpha / 4+\beta / 2+\gamma}\right) \\
& +\log _{2}\left(\# \mathcal{E}_{1 / 16+\alpha^{\prime} / 4+\beta^{\prime} / 2+\gamma^{\prime}, \alpha^{\prime} / 4+\beta^{\prime} / 2+\gamma^{\prime}}^{(3)}\right)-\ell_{3} \log _{2}(q) \\
\approx & m^{\prime} H(1 / 16+\alpha / 4+\beta / 2+\gamma, \alpha / 4+\beta / 2+\gamma) \\
& +\ell_{3} H\left(1 / 16+\alpha^{\prime} / 4+\beta^{\prime} / 2+\gamma^{\prime}, \alpha^{\prime} / 4+\beta^{\prime} / 2+\gamma^{\prime}\right)-\ell_{3} \log _{2}(q) .
\end{aligned}
$$

For our parameters we have the relation

$$
q^{\ell_{3}}=2^{0.2408 m^{\prime}} 2^{0.0507 n}
$$

Hence the logarithmic size is

$$
\begin{aligned}
\log _{2}\left(\# L^{(3)}\right) & =0.2908 m^{\prime}+\frac{0.1120 m^{\prime} n}{m^{\prime}+n}-0.0507 n+\frac{0.0236 n^{2}}{m^{\prime}+n} \\
& \leq 0.2908 m^{\prime}+0.0613 n
\end{aligned}
$$

Using the approximate merge (Algorithm 2), the cost to merge is

$$
T_{3}=\tilde{O}\left(\# L^{(3)} \# L^{(3)} \mathcal{E}_{1 / 8+\alpha^{\prime} / 2+\beta^{\prime}, \alpha^{\prime} / 2+\beta^{\prime}}^{(2)} / q^{\ell_{2}}\right)
$$

With the above parameters

$$
\begin{aligned}
\log _{2}\left(T_{3}\right) & \approx 0.2912 m^{\prime}+\frac{0.4293 m^{\prime} n}{m^{\prime}+n}-0.2402 n+\frac{0.1453 n^{2}}{m^{\prime}+n} \\
& \leq 0.2912 m^{\prime}+0.1891 n
\end{aligned}
$$

Each list in the last level can be constructed using the Shroeppel-Shamir algorithm (or its variants) in time

$$
T_{4}=\tilde{O}\left(\sqrt{\# \mathcal{X}_{1 / 16+\alpha / 4+\beta / 2+\gamma, \alpha / 4+\beta / 2+\gamma} \# \mathcal{E}_{1 / 16+\alpha^{\prime} / 4+\beta^{\prime} / 2+\gamma^{\prime}, \alpha^{\prime} / 4+\beta^{\prime} / 2+\gamma^{\prime}}^{(3)}}\right) .
$$

We have

$$
\begin{aligned}
\log _{3}\left(T_{4}\right) & \approx 0.2658 m^{\prime}+\frac{0.0560 m^{\prime} n}{m^{\prime}+n}+\frac{0.0118 n^{2}}{m^{\prime}+n} \\
& \leq 0.2658 m^{\prime}+0.0560 n .
\end{aligned}
$$


By taking the maximum of the running times of all levels in the recursion, the time complexity of the algorithm is $\tilde{O}\left(2^{0.2912 m^{\prime}+0.1899 n}\right)$.

Remark 3. Note that $\tilde{O}\left(2^{0.2912 m^{\prime}+0.1899 n}\right)<\tilde{O}\left(2^{0.2912 m}\right)$, so the algorithm is an improvement over standard BCJ by a multiplicative factor $2^{0.1013 n}$. If one fixes $n$ and considers $m \rightarrow \infty$ then the success probability is constant and the running time is the same $\tilde{O}\left(2^{0.2912 m}\right)$ statement as given in [4] but with an improved constant.

\section{Improved Attacks on SWIFFT}

We present improved attacks for both inversion and finding collisions of the SWIFFT hash function. The parameters are $m=1024, n=64, q=257$ and $\mathcal{B}=\{0,1\}$. Note that for the moment we ignore the ring structure and just treat SWIFFT as an instance of ISIS. The best previously known attacks for the SWIFFT inversion and collision problems require $2^{148}$ bit operations and $2^{113}$ bit operations respectively. Our improved attacks solve the SWIFFT inversion and collision problems in $2^{138}$ bit operations and $2^{104}$ bit operations respectively. Our results do not violate the claimed security level of SWIFFT.

\subsection{Inverting SWIFFT}

Lyubashevsky, Micciancio, Peikert and Rosen [21] discussed using the original CPW algorithm to solve the $(1024,64,257,\{0,1\})$-SIS problem: "it is also possible to mount an inversion attack using time and space approximately $2^{128}$ ". Note that $2^{128}$ is the initial list size required and hence the running-time would be expected to be larger. We re-estimate the bit-complexity of the algorithm. The method breaks up the 1024 column vectors of matrix A into $k=8$ groups of 128 column vectors each. For each group we compute a list of size $2^{128}$, then choose $\ell_{1}=16, \ell_{2}=16, \ell_{3}=32$. At each level the size of the lists is around $2^{128}$, so the required storage is about $8 \cdot 2^{128} \cdot \log _{2}\left(q^{16}\right)$ bits. Using Lemma 3 of the special case $\gamma_{i}=C_{1}=C_{2}=0$, we predict the total running time is approximately $2^{148}$ bit operations for success probability at least $1 / 2$.

We now show that using the Hermite normal form and our approximate CPW algorithm from Sect. 4.2 gives a $2^{10}$ speedup. First, we lower the dimension from 1024 to 1000 by setting 24 entries of $\mathbf{x}$ to be zero and deleting the corresponding columns from $\mathbf{A}$. This reduces the density, but we still have high enough density to run the CPW algorithm. Then we compute the Hermite normal form, to reduce $\mathbf{A}$ to a $64 \times 936$ matrix. We then use $k=8$ to break $\{0,1\}^{936}$ into 8 groups of length 117 . Thus we construct 8 initial lists of size $2^{117}$ and take $\ell_{1}=15, \ell_{2}=16$ and $\ell_{3}=33$.

At each step, we merge two lists using the "approximate merge" algorithm described in Algorithm 2. To begin, we merge the initial eight lists of size $2^{117}$ by checking the first $\ell_{1}=15$ coordinates of the vectors. We allow errors in $\gamma_{1}=4,4,3,4$ positions for each merge. The expected size of the three new lists corresponding to $\gamma_{1}=4$ is $\frac{2^{2 \cdot 117} \cdot 2^{4}}{257^{15}} \approx 2^{117.92}$, and the expected size of the other list is $\frac{2^{2 \cdot 117} \cdot 2^{3}}{257^{15}} \approx 2^{116.92}$. For hashing, we take $v_{1}=7$ most significant bits of each value in $\mathbb{Z}_{257}$. The probability $p_{\text {flip }} \approx 0.25$ is such that \#Flips $(\mathbf{v})$ is on average no more than 2.5 , and the expected 
number of collisions in a hash slot (i.e., the expected number of $\mathbf{x}$ in a list that have the same value for $F(\mathbf{A x}(\bmod H)))$ is $\frac{2^{117}}{2^{v_{1} \gamma_{1}} q^{\ell_{1}-\gamma_{1}}} \leq 2$.

At the next level we merge the four lists of sizes $2^{117.92}, 2^{117.92}, 2^{116.92}, 2^{117.92}$ by checking the next $\ell_{2}=16$ coordinates of the vectors. We allow errors in $\gamma_{2}=8$ positions for each merge. The expected sizes of the two new lists is $\frac{2^{117.92+117.92} \cdot 2^{8}}{257^{16}} \approx 2^{115.75}$ and $\frac{2^{116.92+117.92} \cdot 2^{8}}{257^{16}} \approx 2^{114.75}$. For the hashing of each merge, we take $v_{2}=7$. The probability $p_{\text {flip }} \approx 0.25$ such that \#Flips $(\mathbf{v}) \approx 6$ and the expected number of elements $\mathrm{n}$ a hash slot is bounded above by 1 .

At the top level we merge the two lists of sizes $2^{114.75}$ and $2^{115.75}$ by checking the remaining $\ell_{3}=33$ coordinates of the vectors, allowing $\gamma_{3}=33$ positions to have errors. The expected size of the solution set ${ }^{8}$ is $\frac{2^{114.75+115.75} 2^{33}}{257^{33}} \approx 2^{-0.7}$. For hashing we use $v_{3}=4$. Thus \#Flips $(\mathbf{v}) \approx 3.1$ and the expected number of elements in a hash slot is bounded above by 1 .

In conclusion, the maximum size of the lists at all levels is $2^{117.92}$. Using the above information and Lemma 3, we estimate the total time to be around $2^{138}$ bit operations (and with a similar success probability as the method of [21] which is about 0.6).

Remark 4. The analysis of the expected size of lists assumes independence of vectors in the lists. However, when the ISIS instance comes from a ring then there are certain symmetries that show that vectors are not independent. We have theoretical and experimental evidence that these issues do not effect the analysis of the CPW algorithm on SWIFFT. See Remark 5 in Sect. 6 for further discussion.

\subsection{Finding Collisions for SWIFFT}

Finding collisions for SWIFFT is equivalent to solving the $(1024,64,257,\{-1,0,1\})$ SIS problem.

Lyubashevsky, Micciancio, Peikert and Rosen [21] give an analysis using the CPW algorithm and choose $k=16$. The method breaks up the 1024 column vectors of $\mathbf{A}$ into 16 groups of 64 vectors each, for each group create an initial list of $3^{64} \approx 2^{101.4}$ vectors. Then we choose $\ell_{1}=\ell_{2}=\ell_{3}=\ell_{4}=13$ to perform the merges. Since the goal of [21] is to provide a lower bound on the running time of the most efficient known algorithm: they optimistically assume that, at each level, the lists have $2^{102}$ vectors. Thus the final level ends up with a list of $\approx 2^{102}$ elements whose first 52 coordinates are all zero. Since $2^{102}>257^{12} \approx 2^{96}$, it is expected that there exists an element whose last 12 coordinates are also zero, which leads to the conclusion that the space is at least $2^{102}$ and the running time is at least $2^{106}$. Kirchner [19] also claims a collision algorithm with similar complexity.

Note that the assumption in [21] that the lists have $2^{102}$ elements at each level seems implausible. In practice, it is useful to obtain a more precise estimate of the running time of the method. In particular, the lists at various levels are getting smaller (sizes

\footnotetext{
${ }^{8}$ Here we tune the constraint $b_{t} \geq 0$ to be $b_{t} \geq-1$, which means we expect to have to run the whole algorithm twice. It is possible to tune certain constraints in the integer program in Sect. 4.2 to get a better attack in terms of bit-complexity.
} 
$2^{101.4} \rightarrow 2^{98.8} \rightarrow 2^{93.5} \rightarrow 2^{83.0} \rightarrow 2^{61.9}$ ) and so one does not have a list of $2^{102}$ vectors at the final level. The success probability (using the expected size on the lists) of this algorithm is around $2^{-34}$. Taking these into consideration, the bit complexity of this approach is about $2^{153}$.

One can already improve the above approach by using Minder and Sinclair's refinement [25]. For $k=16$ lists one can take $\ell_{1}=12, \ell_{2}=14, \ell_{3}=12, \ell_{4}=26$. The maximum size of the lists at all the levels is then around $2^{107}$. Using Lemma 3 we estimate the total time to be about $2^{126}$ bit operations.

Howgrave-Graham and Joux described an improved collision attack in Appendix B of [16] (an early version of [15]). The idea is to attack the original $\{0,1\}$-SIS problem directly: first using the original CPW algorithm to get a list of elements with a certain subset of their coordinates equal to 0 , then exploit the birthday paradox using the elements in this list to find a collision between the remaining coordinates. They choose $k=16$ and create 16 initial lists of size $2^{64}$. By choosing $\ell_{1}=4, \ell_{2}=12, \ell_{3}=12, \ell_{4}=12$, the size of the lists on all other levels (except the initial and the solution lists) is about $2^{96}$. In the final step they obtain a list of $2^{96}$ elements with the first 40 coordinates equal to zero. Since $\left(2^{96}\right)^{2} \approx 257^{24}$, the birthday paradox shows one can find a collision between the remaining 24 coordinates in this list. In other words, we have $\mathbf{A} \mathbf{x}_{1} \equiv \mathbf{A} \mathbf{x}_{2}$ where $\mathbf{x}_{1}, \mathbf{x}_{2} \in\{0,1\}^{m}$ and so we have found a collision for SWIFFT. The space requirement is about $2^{96}$ and the time is predicted in [16] to be proportional to $2^{100}$. Lemma 3 suggests the total bit-operation is about $2^{115}$, thus a speedup of $2^{11}$ from the aforementioned Minder-Sinclair method. The success probability is about $2^{-1.4}$

We now describe a better collision attack, by using the Hermite normal form and the approximate-CPW algorithm from Sect. 4.2. We apply the HNF to have an $n \times m^{\prime}$ instance, where $m^{\prime}=m-n=960$. Then we use the CPW method (with Minder and Sinclair's refinement) to construct a list of $\mathbf{x} \in\{0,1\}^{960}$ such that $\mathbf{A x}(\bmod H)$ has coordinates lying in $\{-1,0\}$ (in other words, there is a binary error vector $\mathbf{e}$ such that $\mathbf{A x}+\mathbf{e} \equiv \mathbf{0}(\bmod H))$. In the end, we exploit the birthday paradox to find a near collision between the remaining coordinates: the "near collision" means that the difference of the coordinates lies in $\{-1,0,1\}$.

Let $k=16$ and we divide the matrix into 16 groups of 60 vectors each. For each group create an initial list. Note that we can control the size of the initial lists and they do not need to have the same size. We choose $\ell_{1}=5, \ell_{2}=10, \ell_{3}=11, \ell_{4}=12$ to perform our approximate merge. These values can be obtained by solving the integer program described in Sect. 4.2: one needs to modify the constraint $b_{t} \geq 0$ (one solution survives at the bottom level) in the integer program to $2 b_{t}+\log _{2}(3) \cdot\left(n-\sum_{i=1}^{t} \ell_{i}\right) \geq$ $\log _{2}(q) \cdot\left(n-\sum_{i=1}^{t} \ell_{i}\right)$, i.e., in the last level we want the size of the list to be large enough to use the birthday paradox. As long as this size is sufficiently large, there exist two elements (a near collision) $\mathbf{x}_{1}, \mathbf{x}_{2}$ such that $\mathbf{A}\left(\mathbf{x}_{1}-\mathbf{x}_{2}\right)$ has its remaining coordinates coming from $\{-1,0,1\}$. Figure 2 shows the size of the lists in each level and other parameters. In the last level, we obtain a list of $2^{83.45}$ elements with 38 coordinates equal to $\{-1,0\}$. Since $2^{83.45+83.45} 3^{26} \approx 257^{26}$, a list of size $2^{83.45}$ large enough to use the birthday paradox on the remaining 26 coordinates.

In the above procedure, the maximum list size is $2^{83.88}$ and hence the space is proportional to $2^{84}$. By Lemma 3 the total running time is estimated to be $2^{104}$ bit operations; hence leads to a $2^{11}$ speedup over the previous best method. Compared to previous 


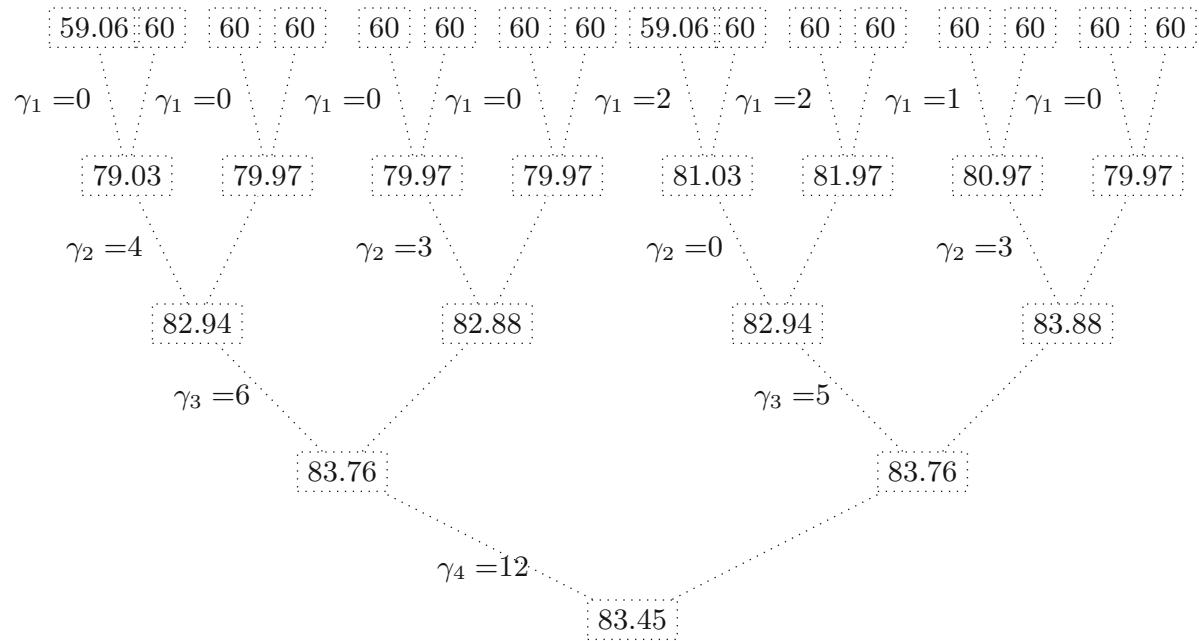

Fig. 2. Parameter choices and list sizes for the approximate-CPW algorithm for finding collisions in SWIFFT. As stated above, we take $\ell_{1}=5, \ell_{2}=10, \ell_{3}=11$ and $\ell_{4}=12$. The values $v_{i}$, being the number of most significant bits we use for the hash in the cases when $\gamma_{i} \neq 0$, are $v_{1}=v_{2}=8$ and $v_{3}=v_{4}=7$. The numbers in the dotted box denote the $\log _{2}$ size of the list; the $\gamma_{i}$ is used in the approximate-merge algorithm.

methods, we start with much smaller lists due to HNF and use the approximate merge to increase the list size. This helps to control the maximum list sizes during the procedure.

\section{Ring-SIS}

We consider the ring $R_{q}=\mathbb{Z}_{q}[t] /\left(t^{n}+1\right)$, where $q$ is prime and $n$ is typically a power of 2. For example, the SWIFFT hash function is defined using $q=257$ and $n=64$. Ring elements are often represented as tuples $\left(a_{0}, \ldots, a_{n-1}\right) \in \mathbb{Z}_{q}^{n}$ corresponding to the polynomial $a_{0}+a_{1} t+\cdots+a_{n-1} t^{n-1}$. Define a subset $\mathcal{X}$ of $R_{q}$ as those polynomials with coefficients $a_{i} \in\{0,1\}$, or possibly $\{-1,0,1\}$. The Ring-SIS problem is, given $\mathbf{a}_{1}, \ldots, \mathbf{a}_{m} \in R_{q}$, to find $\mathbf{x}_{1} \ldots, \mathbf{x}_{m} \in \mathcal{X}$ such that $\mathbf{0}=\mathbf{a}_{1} \mathbf{x}_{1}+\cdots+\mathbf{a}_{m} \mathbf{x}_{m}$ and not all $\mathbf{x}_{i}=0$. The SWIFFT hash function uses $m=16$, giving $2^{m n}=2^{1024}$ choices for $\left(\mathbf{x}_{1}, \ldots, \mathbf{x}_{m}\right)$. One can find collisions in the SWIFFT hash function by solving Ring-SIS with the set $\mathcal{X}$ being polynomials with coefficients $a_{i} \in\{-1,0,1\}$.

All previous cryptanalytic work on the SWIFFT hash function ignored the ring structure and converted the problem to (I)SIS. In this section we consider exploiting the ring structure to obtain a speedup. Our tool is the map $\psi: R_{q} \rightarrow R_{q}$ given by $\psi(a(t))=\operatorname{ta}(t)$. This is not a ring homomorphism. We will think of $\psi$ as an invertible linear map on the $\mathbb{Z}_{q}$-vector space $R_{q}$, and call it a "symmetry" (technically, we have a group action). In terms of coefficient vectors, we have

$$
\psi\left(a_{0}, \ldots, a_{n-1}\right)=\left(-a_{n-1}, a_{0}, \ldots, a_{n-2}\right) .
$$


Note that $\psi$ has order $2 n$ and that $\psi^{n}=-1$.

The key observation is that

$$
\psi(\mathbf{a x})=\psi(a(t) x(t))=\operatorname{ta}(t) x(t)=a(t)(t x(t))=\mathbf{a} \psi(\mathbf{x}) .
$$

Hence, an orbit $\left\{\psi^{i}(\mathbf{a x}): 0 \leq i<2 n\right\}$ can be considered as a times the orbit of $\mathbf{x}$. If the set $\mathcal{X}$ has the property that $\psi(\mathcal{X})=\mathcal{X}$ then one can adapt the CPW algorithm to work with the set of orbits $\mathcal{X} / \psi$ rather than the whole set $\mathcal{X}$. The hope is that this gives a reduction by a factor $2 n$ in the cost of all stages of the algorithm.

For the parameters in SWIFFT we do not have $\psi(\mathcal{X})=\mathcal{X}$. This is because $\mathcal{X}$ consists of polynomials with coefficients in $\{0,1\}$, whereas $t^{64}=-1$ and so large enough shifts of polynomials in $\mathcal{X}$ have some coefficients equal to -1 . So for the remainder of this section we restrict attention to cases where $\psi(\mathcal{X})=\mathcal{X}$. This is the case when the ring $R_{q}$ is defined as $\mathbb{Z}_{q}[t] /\left(t^{n}-1\right)$, or if $\mathcal{X}$ is the set of polynomials with coefficients in $\{-1,0,1\}$.

A remaining non-trivial issue is how to deal with quotients $G / H$. Our group $G$ is now the additive group of the ring $R_{q}$, and to have a well-defined action of $\psi$ on the quotient $G / H$ it is necessary that $H$ be invariant under $\psi$ (i.e., $\psi(H)=H$ ). It might seem that this condition greatly restricts our possible choices for $H$, and so does not allow arbitrary fine-tuning of the size of $G / H$. We now explain that for the parameters used in SWIFFT no such obstruction arises.

The matrix representation of $\psi$ with respect to the basis $\left\{1, t, \ldots, t^{n-1}\right\}$ is

$$
\left(\begin{array}{ccccc}
0 & 1 & 0 & \ldots & 0 \\
0 & 0 & 1 & \ldots & 0 \\
\vdots & \vdots & \vdots & & \vdots \\
0 & 0 & 0 & \ldots & 1 \\
-1 & 0 & 0 & \cdots & 0
\end{array}\right)
$$

One can verify that, when $q=257$ and $n=64$, this matrix has $n$ distinct eigenvalues (specifically, the 64 distinct 128 th roots of 1 in $\left.\mathbb{Z}_{257}^{*}\right)$. This happens since $128 \mid(257-1)$; a choice that was taken so that $t^{64}+1$ splits completely modulo $q$ (which enables fast computation of the hash function using the number theoretic transform). This means that there is an eigenbasis for $\psi$. With respect to this basis of $\mathbb{Z}_{257}^{64}$ the matrix representing $\psi$ is diagonal. Hence, by taking $H$ to be the group generated by any $64-\ell$ of these eigenvectors we have a $\psi$-invariant subgroup such that $\#(G / H)=q^{\ell}$. On the other hand, if $t^{n}+1$ did not split completely modulo $q$ then the attacker's job would likely be a little more difficult.

We now very briefly describe the CPW algorithm in the case when $\mathcal{X}$ consists of polynomials with coefficients in $\{-1,0,1\}$. Suppose one wants to solve $\mathbf{0}=\mathbf{a}_{1} \mathbf{x}_{1}+\cdots+$ $\mathbf{a}_{16} \mathbf{x}_{16}$ using an 8-list algorithm. The original CPW algorithm would choose a suitable subgroup $H_{1}$ and compute lists $L_{i}^{(0)}=\left\{\left(\mathbf{a}_{2 i-1} \mathbf{x}_{2 i-1}+\mathbf{a}_{2 i} \mathbf{x}_{2 i}\left(\bmod H_{1}\right), \mathbf{x}_{2 i-1}, \mathbf{x}_{2 i}\right)\right.$ : $\left.\mathbf{x}_{2 i-1}, \mathbf{x}_{2 i} \in \mathcal{X}\right\}$ for $1 \leq i \leq 8$. The size of each set $L_{i}^{(0)}$ is roughly $\# \mathcal{X}^{2}$. One then merges the sets by finding matches $\left(\mathbf{a}_{1} \mathbf{x}_{1}+\mathbf{a}_{2} \mathbf{x}_{2}\left(\bmod H_{1}\right), \mathbf{x}_{1}, \mathbf{x}_{2}\right) \in L_{1}^{(0)}$ and $\left(\mathbf{a}_{3} \mathbf{x}_{3}+\mathbf{a}_{4} \mathbf{x}_{4}\right.$ $\left.\left(\bmod H_{1}\right), \mathbf{x}_{3}, \mathbf{x}_{4}\right) \in L_{2}^{(0)}$ such that $\mathbf{a}_{1} \mathbf{x}_{1}+\mathbf{a}_{2} \mathbf{x}_{2}+\mathbf{a}_{3} \mathbf{x}_{3}+\mathbf{a}_{4} \mathbf{x}_{4} \equiv \mathbf{0}\left(\bmod H_{1}\right)$. Our 
observation is that we also have $\left(\psi^{i}\left(\mathbf{a}_{1} \mathbf{x}_{1}+\mathbf{a}_{2} \mathbf{x}_{2}\right)\left(\bmod H_{1}\right), \psi^{i}\left(\mathbf{x}_{1}\right), \psi^{i}\left(\mathbf{x}_{2}\right)\right) \in L_{1}^{(0)}$ and $\left(\psi^{i}\left(\mathbf{a}_{3} \mathbf{x}_{3}+\mathbf{a}_{4} \mathbf{x}_{4}\right)\left(\bmod H_{1}\right), \psi^{i}\left(\mathbf{x}_{3}\right), \psi^{i}\left(\mathbf{x}_{4}\right)\right) \in L_{2}^{(0)}$, and these values satisfy

$$
\psi^{i}\left(\mathbf{a}_{1} \mathbf{x}_{1}+\mathbf{a}_{2} \mathbf{x}_{2}\right)+\psi^{i}\left(\mathbf{a}_{3} \mathbf{x}_{3}+\mathbf{a}_{4} \mathbf{x}_{4}\right) \equiv \mathbf{0} \quad\left(\bmod H_{1}\right)
$$

Hence the idea is to store a single representative of each orbit. We now define the representative we use.

Definition 3. Let notation be as above, with $q$ prime and $2 n \mid(q-1)$. Let $k=$ $(q-1) /(2 n)$ and let $\alpha_{1}, \ldots, \alpha_{k}$ be coset representatives for $\mathbb{Z}_{q}^{*} / Z$ where $Z=\left\{z \in \mathbb{Z}_{q}^{*}\right.$ : $\left.z^{2 n}=1\right\}$. Suppose $\psi$ has distinct eigenvalues $\lambda_{1}, \ldots, \lambda_{n}$ so that, with respect to the corresponding eigenbasis, $\psi\left(\left(s_{1}, \ldots, s_{n}\right)^{T}\right)=\left(s_{1} \lambda_{1}, \ldots, s_{n} \lambda_{n}\right)^{T}$. Let $\mathbf{s} \in R_{q} / H$ for any subgroup $H$ such that $\psi(H)=H$. Then the equivalence class representative [s] of $\mathbf{s}$ is defined to be the element of $\left\{\psi^{i}(\mathbf{s}): 0 \leq i<2 n\right\}$ such that $[\mathbf{s}]=\left(0, \ldots, 0, \alpha_{j}, \star, \ldots, \star\right)$ for some $1 \leq j \leq k$ and $\star$ denotes an arbitrary element.

Note that $[\mathbf{s}]=\left(s_{1} \lambda_{1}^{i}, s_{2} \lambda_{2}^{i}, \ldots, s_{n} \lambda_{n}^{i}\right)^{T}$ for some $0 \leq i<2 n$. Writing $\lambda_{j}=\lambda_{1}^{a_{j}}$ one can write this as

$$
\left(s_{1} \lambda_{1}^{i}, s_{2} \lambda_{1}^{a_{2} i}, \ldots, s_{n} \lambda_{1}^{a_{n} i}\right)^{T}
$$

To compute the class representative efficiently one uses a precomputed table of discrete logarithms with respect to $\lambda_{1}$. In the case of SWIFFT we have $k=2$ and can take $\alpha_{1}=1$ and $\alpha_{2}=3$.

Returning to the CPW algorithm. The idea is to store a single representative of each class, so that the list $L_{1}^{(0)}$ becomes $\left\{\left(\left[\mathbf{a}_{1} \mathbf{x}_{1}+\mathbf{a}_{2} \mathbf{x}_{2}\left(\bmod H_{1}\right)\right], \mathbf{x}_{1}, \mathbf{x}_{2}\right): \mathbf{x}_{1}, \mathbf{x}_{2} \in \mathcal{X}\right\}$. We store the specific pair $\left(\mathbf{x}_{1}, \mathbf{x}_{2}\right)$ such that $\left[\mathbf{a}_{1} \mathbf{x}_{1}+\mathbf{a}_{2} \mathbf{x}_{2}\left(\bmod H_{1}\right)\right]=\mathbf{a}_{1} \mathbf{x}_{1}+\mathbf{a}_{2} \mathbf{x}_{2}$ ( $\bmod H_{1}$ ). We now consider the merge operation. The question is how to find all tuples $\left(\mathbf{x}_{1}, \mathbf{x}_{2}, \mathbf{x}_{3}, \mathbf{x}_{4}\right)$ such that

$$
\mathbf{a}_{1} \mathbf{x}_{1}+\mathbf{a}_{2} \mathbf{x}_{2}+\mathbf{a}_{3} \mathbf{x}_{3}+\mathbf{a}_{4} \mathbf{x}_{4} \equiv \mathbf{0} \quad\left(\bmod H_{1}\right)
$$

when given only the classes $\left[\mathbf{a}_{1} \mathbf{x}_{1}+\mathbf{a}_{2} \mathbf{x}_{2}\left(\bmod H_{1}\right)\right],\left[\mathbf{a}_{3} \mathbf{x}_{3}+\mathbf{a}_{4} \mathbf{x}_{4}\left(\bmod H_{1}\right)\right]$. In other words, we need to determine all indices $i, i^{\prime}$ such that

$$
\psi^{i}\left(\mathbf{a}_{1} \mathbf{x}_{1}+\mathbf{a}_{2} \mathbf{x}_{2} \quad\left(\bmod H_{1}\right)\right) \equiv \psi^{i^{\prime}}\left(-\left(\mathbf{a}_{3} \mathbf{x}_{3}+\mathbf{a}_{4} \mathbf{x}_{4} \quad\left(\bmod H_{1}\right)\right)\right)
$$

Lemma 4. Suppose a quadruple ( $\left.\boldsymbol{x}_{1}, \boldsymbol{x}_{2}, \boldsymbol{x}_{3}, \boldsymbol{x}_{4}\right)$ satisfies Eq. (10). Then $\left[\boldsymbol{a}_{1} \boldsymbol{x}_{1}+\boldsymbol{a}_{2} \boldsymbol{x}_{2}\right.$ $\left.\left(\bmod H_{1}\right)\right]=\left[\boldsymbol{a}_{3} \boldsymbol{x}_{3}+\boldsymbol{a}_{4} \boldsymbol{x}_{4}\left(\bmod H_{1}\right)\right]$. Furthermore, if $\left[\boldsymbol{a}_{1} \boldsymbol{x}_{1}+\boldsymbol{a}_{2} \boldsymbol{x}_{2}\left(\bmod H_{1}\right)\right]=$ $\boldsymbol{a}_{1} \boldsymbol{x}_{1}+\boldsymbol{a}_{2} \boldsymbol{x}_{2}\left(\bmod H_{1}\right)$ and $\left[\boldsymbol{a}_{3} \boldsymbol{x}_{3}+\boldsymbol{a}_{4} \boldsymbol{x}_{4}\left(\bmod H_{1}\right)\right]=\boldsymbol{a}_{3} \boldsymbol{x}_{3}+\boldsymbol{a}_{4} \boldsymbol{x}_{4}\left(\bmod H_{1}\right)$ then $i^{\prime}=i$.

Proof. Without loss of generality we can apply a suitable power of $\psi$ to both sides, so that the left hand side equals $\left[\mathbf{a}_{1} \mathbf{x}_{1}+\mathbf{a}_{2} \mathbf{x}_{2}\left(\bmod H_{1}\right)\right]$, and so is a normalized vector $\left(0, \cdots, 0, \alpha_{j}, \star, \cdots\right)^{T}$. The right hand side is then $\psi^{i \prime \prime}\left(-\left(\mathbf{a}_{3} \mathbf{x}_{3}+\mathbf{a}_{4} \mathbf{x}_{4}\left(\bmod H_{1}\right)\right)\right)$ for 
some integer $i^{\prime \prime}$. To get equality it follows that the right hand side is also a normalized vector, and so

$$
\psi^{i^{\prime \prime}}\left(-\left(\mathbf{a}_{3} \mathbf{x}_{3}+\mathbf{a}_{4} \mathbf{x}_{4} \quad\left(\bmod H_{1}\right)\right)\right)=\left[-\left(\mathbf{a}_{3} \mathbf{x}_{3}+\mathbf{a}_{4} \mathbf{x}_{4} \quad\left(\bmod H_{1}\right)\right)\right] .
$$

The result follows.

It follows that all matches in the merge algorithm can be found by simply checking equality of equivalence class representatives. Hence, the merge algorithm is exactly the same as Algorithm 1.

It follows that the sizes of lists are reduced from $3^{n}$ to roughly $3^{n} /(2 n)$ and that the merge time is reduced correspondingly. There are some additional challenges in programming the algorithm, but they should not lead to a slower program overall.

Remark 5. We now make a remark about success probability. The original CPW algorithm would have lists of size $3^{n}$, and they would be merged by checking a condition in $G / H=\mathbb{Z}_{q}^{\ell}$. The heuristic argument is that the merged list would be of size roughly $\left(3^{n}\right)^{2} / q^{\ell}$, and hence consist of approximately $3^{2 n} /\left(2 n q^{\ell}\right)$ orbits under $\psi$. In our new algorithm we have lists of size $3^{n} /(2 n)$ and, by Lemma 4 , there is a unique match (namely, it is sufficient to test equality of the normalized class representatives as in Definition 3 ). Since the number of normalized class representatives is roughly $q^{\ell} /(2 n)$ it follows that the size of the new list (whose entries are class representatives) should be approximately $\left(3^{n} / 2 n\right)^{2} /\left(q^{\ell} / 2 n\right) \approx 3^{2 n} /\left(2 n q^{\ell}\right)$, which is consistent with the original algorithm. This argument supports our claim in Remark 4 (Sect. 5.1) that the symmetries do not negatively impact the success probability of these algorithms when applied to structured matrices.

These ideas do not lead to a better attack on SWIFFT for two reasons:

1. It seems hard to use this idea for Ring-ISIS (and hence inverting SWIFFT) because $\psi$ does not fix the inhomogenous term s. Also, in that case we would not have the required condition $\psi(\mathcal{X})=\mathcal{X}$.

Instead, the main application seems to be finding collisions in SWIFFT by solving Ring-SIS.

2. We already have a speedup to the CPW algorithm by a factor of more than $2 n$, by using the Hermite normal form and other ideas.

It is an open problem to combine the use of orbits under $\psi$ with the Hermite normal form. We briefly explain the issue. Solving Ring-SIS is finding $\mathbf{x}_{1}, \ldots, \mathbf{x}_{16}$ such that $\mathbf{0}=\mathbf{a}_{1} \mathbf{x}_{1}+\cdots+\mathbf{a}_{16} \mathbf{x}_{16}$. Assuming $\mathbf{a}_{16} \in R_{q}$ is invertible, the Hermite normal form is

$$
\mathbf{0}=\left(\mathbf{a}_{16}^{-1} \mathbf{a}_{1}\right) \mathbf{x}_{1}+\cdots+\left(\mathbf{a}_{16}^{-1} \mathbf{a}_{15}\right) \mathbf{x}_{15}+\mathbf{x}_{16}
$$

One then wants to perform an 8-list approximate-CPW algorithm on the sum of 15 elements. The problem is that 8 does not divide 15 , so when we split the sum of 15 terms into 8 blocks, we are unable to get a clean division into a sum of ring elements. Of course, we can discard the ring structure and split the sum as we did in Sect. 5.1. But 
since blocks no longer correspond to ring elements, we do not have an action by $\psi$ and hence do not seem to be able to achieve any speedup.

\section{Experimental Results}

The purpose of this section is three-fold. First, we check the heuristic assumptions used in the analysis. Second, we show that the improved algorithms achieve the predicted speedup in practice. Third, we show that our time and space complexity estimates are robust: the actual running-time of the algorithm follows from the bit complexity (and hence size) estimate.

For our experiments with the SWIFFT inversion and collision problems we study a version of SWIFFT with smaller parameters. We consider two scenarios: the SIS inversion problem with $\mathcal{B}=\{0,1\}$ and the SIS collision problem with $\mathcal{B}=\{0,1\}$.

\subsection{On Our Heuristic Assumptions}

In this subsection, we experimentally investigate the heuristic assumptions used in the paper. We use the SAGE software for the implementation and the experiments. For this part, we run the experiments on a cluster of Intel Xeon X5650 2.67GHz cores.

One of the main heuristic assumptions used in the analysis is Heuristic 1. It is used to show that the expected size of a merged list is determined by the sizes of the two input lists. Theoretical evidence for this heuristic comes from regularity arguments in $[21,22]$. However, the splitting of the columns in the matrix A may not follow this theoretical argument. Furthermore, we need to invoke this assumption at all levels and any discrepancy could accumulate, and this could lead to problems in the overall estimate. Thus we check the assumption by performing experiments for all levels. Rather than testing the heuristic directly we test its impact on the algorithm (the sizes of lists after merging), since this is actually what is important for our claims. In the experiments, we work with the ring $R_{q}=\mathbb{Z}_{q}[t] /\left(t^{n}+1\right)$, where $q$ is prime and $n$ is typically a power of 2 . We also check several sets of parameters which requires different numbers of levels in the procedure.

In the first set of parameters, we take $n=16, m=112$ and $q=13$. We generate 1000 instances of the ring-ISIS inversion problem for $\mathcal{B}=\{0,1\}$ where each ring element $\mathbf{a}_{i}$ is uniformly and independently generated. Then we form $\mathbf{A}$ by concatenating the anti-circulant matrices representing all $\mathbf{a}_{i}$ 's. For the solving algorithm, we use MinderSinclair's variant of CPW. We use three levels (thus 8 initial lists) with $\ell_{1}=3, \ell_{2}=5$ and $\ell_{3}=8$. The lists size for all levels are tabulated in Table 4. These results show an extremely close agreement between theory and practice and so emphatically confirm that our heuristics are reasonable.

We explain the notation: The rows $\log _{2}\left(\# L^{(i)}\right)$ give the logarithm of the average size of the lists at the $i$ th level in our experiments. The cells/columns under " $\log _{2}$ of list size" give the size of the lists at various levels of the algorithm: thus we start with 8 lists and end with 1 list of solutions. Each cell corresponds to a list which is merged from the two cells (lists) on the top of the current cell (e.g., the list with log. size 15.30 is merged 
Table 4. List size for parameters $n=16, m=112$ and $q=13$.

\begin{tabular}{|c|c|c|c|c|c|}
\hline Levels & \multicolumn{4}{|c|}{$\log _{2}$ of list size } & Init. Est \\
\hline $\log _{2}\left(\# L^{(0)}\right)$ & \begin{tabular}{|l|l|}
14.00 & 14.00 \\
\end{tabular} & \begin{tabular}{|l|l|l|}
5.00 & 14.00
\end{tabular} & \begin{tabular}{|l|l|}
14.00 & 14.00 \\
\end{tabular} & \begin{tabular}{|l|l|}
14.00 & 14.00 \\
\end{tabular} & 14.00 \\
\hline $\log _{2}\left(\# L^{(1)}\right)$ & 16.90 & 16.90 & 16.90 & 16.90 & 16.90 \\
\hline $\log _{2}\left(\# L^{(2)}\right)$ & \multicolumn{2}{|c|}{15.30} & \multicolumn{2}{|c|}{15.30} & 15.30 \\
\hline $\log _{2}\left(\# L^{(3)}\right)$ & \multicolumn{4}{|c|}{1.06} & 0.99 \\
\hline
\end{tabular}

Table 5. List size for parameters $n=32, m=1024$ and $q=13$.

\begin{tabular}{|c|c|c|c|c|c|c|c|c|c|}
\hline Levels & \multicolumn{8}{|c|}{$\log _{2}$ of lists size } & Init. Est \\
\hline $\log _{2}\left(\# L^{(0)}\right)$ & \multicolumn{8}{|c|}{64 initial lists of log. size 16.00 each } & \\
\hline $\log _{2}\left(\# L^{(1)}\right)$ & \begin{tabular}{|l|l|}
17.20 & 17.20 \\
\end{tabular} & \begin{tabular}{|l|l|}
17.20 & 17.20 \\
\end{tabular} & \begin{tabular}{|l|l|}
17.20 & 17.20 \\
\end{tabular} & \begin{tabular}{|l|l|}
17.20 & 17.20 \\
\end{tabular} & \begin{tabular}{|l|l|}
17.20 & 17.20 \\
\end{tabular} & \begin{tabular}{|l|l|}
17.20 & 17.20 \\
\end{tabular} & \begin{tabular}{|l|l|}
17.20 & 17.20 \\
\end{tabular} & \begin{tabular}{|l|l|l|}
17.20 & 17.20 \\
\end{tabular} & \multirow{2}{*}{$\begin{array}{l}16.00 \\
17.20\end{array}$} \\
\hline (continue.) & $17.20 \mid 17.20$ & $17.20 \mid 17.20$ & $17.20 \mid 17.20$ & $17.20 \mid 17.20$ & 17.2017 .20 & $17.20 \mid 17.20$ & $17.20 \mid 17.20$ & $17.20 \mid 17.20$ & \\
\hline $\log _{2}\left(\# L^{(2)}\right)$ & \begin{tabular}{|l|l|}
15.89 & 15.90 \\
\end{tabular} & 15.8915 .90 & \begin{tabular}{|l|l|}
15.89 & 15.89 \\
\end{tabular} & \begin{tabular}{|l|l|}
15.89 & 15.89 \\
\end{tabular} & \begin{tabular}{|l|l|}
15.90 & 15.90 \\
\end{tabular} & \begin{tabular}{|l|l|}
15.89 & 15.89 \\
\end{tabular} & \begin{tabular}{|l|l|}
15.89 & 15.89 \\
\end{tabular} & 15.9015 .89 & 15.89 \\
\hline $\log _{2}\left(\# L^{(3)}\right)$ & 16.99 & 16.99 & 16.99 & 16.99 & 16.99 & 16.99 & 16.99 & 16.99 & 16.99 \\
\hline $\log _{2}\left(\# L^{(4)}\right)$ & \multicolumn{2}{|c|}{19.17} & \multicolumn{2}{|c|}{19.17} & \multicolumn{2}{|c|}{19.17} & \multicolumn{2}{|c|}{19.17} & 19.17 \\
\hline $\log _{2}\left(\# L^{(5)}\right)$ & \multicolumn{4}{|c|}{$\frac{1}{16.14}$} & \multicolumn{4}{|c|}{16.14} & 16.14 \\
\hline $\log _{2}\left(\# L^{(6)}\right)$ & \multicolumn{8}{|c|}{-1.03} & -1.02 \\
\hline
\end{tabular}

from two lists of log. size 16.90). The last column "Init. Est" denotes the theoretical estimations based on the initial list size.

In the second experiment we take slightly larger parameters $n=32, m=1024$ and $q=13$. The purpose is to show that the heuristics are still plausible even when there are more levels. This requires to use a larger number of lists, with 64 initial lists of log. size 16. As before, we generate 1000 instances of the ring-ISIS problem. The list sizes for all levels are tabulated in Table 5. The notation used is the same as in Table 4. The subgroups are defined with $\ell_{i}=4,5,4,4,6,9$ for $i=1$ to 6 . For the last list, the figure -1.03 means that on average there is one solution out of two experiments. This is not a problem since we would run the algorithm twice.

In the third experiment we take a different set of parameters $n=16, m=128$ and $q=17$ and we now use the HNF and the approximate merge algorithm. We generate 1000 instances of the ring-ISIS problem. The purpose is to show that the heuristics on the list sizes are still plausible when the errors are considered. Note that after the HNF we have $m^{\prime}=112$. We use 8 initial lists, each of size $2^{14}$; we also use subgroups defined by $\ell_{1}=4, \ell_{2}=3$ and $\ell_{3}=9$. The lists size for all levels are tabulated in Table 6 . The notation is similar to the previous two tables, except that we also give information on the error lengths $\gamma_{i, j}$ in the table. ${ }^{9}$ The rows Est. $\log _{2}\left(\# L^{(i)}\right)$ denote the estimated logarithmic average list size at the $i$ th level where the estimation is computed using the actual list size at the $(i-1)$ th level.

Furthermore, for the same experiment, we also compute the experimental average number of \#Flips(v) in Lemma 3 (we record the total number of flips checked during the approximate merge algorithm and then average the number by the size of list). The errors used in the approximate merge of Table 6 are $\{2,1,1,1,2,9\}$ respectively (they correspond in order to cells "Errors $\gamma_{i, j}$ " of Table 6 but we did not consider the one without errors). The row "Lmsb" denotes the number of most significant bits used

\footnotetext{
${ }^{9}$ In Sect. 4.2 we assume $\gamma_{i}=\ell_{i} / 2^{t-i}$ are integers. In practice, the values of $\gamma_{i}$ are computed from a integer program. Hence we use the notation $\gamma_{i, j}$ to indicate that the error lengths within the $i$ th level can be different.
} 
Table 6. List size for parameters $n=16, m=128$ and $q=17$.

\begin{tabular}{|c|c|c|c|c|}
\hline Levels & \multicolumn{4}{|c|}{$\log _{2}$ of lists size } \\
\hline $\log _{2}\left(\# L^{(0)}\right)$ & \begin{tabular}{|l|l|}
14.00 & 14.00 \\
\end{tabular} & \begin{tabular}{|l|l|}
14.00 & 14.00 \\
\end{tabular} & \begin{tabular}{|l|l|}
14.00 & 14.00 \\
\end{tabular} & \begin{tabular}{|l|l|l|}
0 & 14.00 & 14.00 \\
\end{tabular} \\
\hline Errors $\gamma_{1, j}$ & 2 & 1 & 0 & 1 \\
\hline Est. $\log _{2}\left(\# L^{(1)}\right)$ & 13.65 & 12.65 & 11.65 & 12.65 \\
\hline $\log _{2}\left(\# L^{(1)}\right)$ & 13.65 & 12.65 & 11.65 & 12.65 \\
\hline Errors $\gamma_{2, j}$ & \multicolumn{2}{|c|}{1} & \multicolumn{2}{|r|}{2} \\
\hline Est. $\log _{2}\left(\# L^{(2)}\right)$ & \multicolumn{2}{|c|}{15.04} & \multicolumn{2}{|c|}{14.04} \\
\hline $\log _{2}\left(\# L^{(2)}\right)$ & \multicolumn{2}{|c|}{15.04} & \multicolumn{2}{|c|}{14.04} \\
\hline Errors $\gamma_{3, j}$ & \multicolumn{4}{|c|}{9} \\
\hline Est. $\log _{2}\left(\# L^{(3)}\right)$ & \multicolumn{4}{|c|}{1.29} \\
\hline $\log _{2}\left(\# L^{(3)}\right)$ & \multicolumn{4}{|c|}{1.40} \\
\hline
\end{tabular}

Table 7. The expected number of \#Flips(v) in experiments of Table 6.

\begin{tabular}{lllllll}
\hline Errors $\gamma_{i, j}$ & 2 & 1 & 1 & 1 & 2 & 9 \\
Lmsb & 4 & 3 & 3 & 4 & 5 & 3 \\
Theoretical average \#Flips $(\mathbf{v})$ & 2.339 & 1.294 & 1.294 & 2.000 & 4.000 & 10.180 \\
Experimental average \#Flips(v) & 2.339 & 1.294 & 1.294 & 2.000 & 4.000 & 10.212
\end{tabular}

for each merge. We record the experimental average numbers of \#Flips(v) for each corresponding merge and compare them to the theoretical values. The theoretical values are computed using the formula $\left(p_{\text {flip }}+1\right)^{\gamma_{i}}$ for the expected value of \#Flips $(\mathbf{v})$ (Table 7).

These experimental results show that the list size estimates and the expected number of flips \#Flips(v) are accurate and the heuristic assumptions are plausible for both the CPW (with Minder-Sinclair's refinement) and the HNF cases.

\subsection{ISIS Inversion $\mathcal{B}=\{0,1\}$}

The parameters here are $n=16, q=11$ and $m$ ranges from 80 to 160 . We compared the extended $k$-tree algorithm (Minder-Sinclair variant of CPW) with our HNF improvement. We tried 1000 instances for each set of parameters starting with different random seeds. Table 8 shows the running-time comparison of algorithms in five sets of parameters. In general, the problems get easier as the density increases and thus the instances are getting easier when $m$ increases.

Experiment E1 denotes the extended $k$-tree (CPW) algorithm of Minder and Sinclair (see Sect. 2.6). Experiment E2 denotes the same algorithm, but with the HNF improvement and using approximate merge (see Sect. 4.2). Columns "\#E" are the theoretical estimate of the maximum number of bits of storage used at any stage of the algorithm during experiment $\mathbf{E}$ respectively. The value $\tilde{m}$ in the Column " $\tilde{m}, \ell_{i}(\mathbf{E})$ " denotes the actual dimension we used (since for a given dimension $m$, it is sometimes better to reduce the dimension to get a faster attack). ${ }^{10}$ The notation $\ell_{i}$ denotes the constraints for each level in the computation; when there are 3 (respectively 4) values $\ell_{i}$ listed it means we are performing an 8-list (respectively 16-list) algorithm. Column "T. E1 ; E2" denotes

\footnotetext{
${ }^{10}$ This does not occur in Table 8, but we see it in Table 9. When the dimension can be reduced to an instance which has been investigated previously, we do not repeat the experiment but just reproduce the experimental results from the previous instance.
} 
Table 8. Comparison of algorithms for ISIS inversion $\mathcal{B}=\{0,1\}$.

\begin{tabular}{llllllll}
\hline$m$ & $\tilde{m}$ and $\ell_{i}(\mathbf{E} 1)$ & $\tilde{m}$ and $\ell_{i}(\mathbf{E 2})$ & $\# \mathbf{E 1}$ & \#E2 & T. E1 / E2 = ratio & T1/T2 & succ. E1 ; E2 \\
\hline $\mathbf{8 0}$ & $80,(1,4,11)$ & $80,(1,4,11)$ & 19.24 & 14.24 & $646.32 \mathrm{~s} / 17.29 \mathrm{~s}=37.38$ & 21.15 & $73.5 \% ; 74.6 \%$ \\
$\mathbf{9 6}$ & $96,(2,5,9)$ & $96,(2,5,9)$ & 17.08 & 14.08 & $93.29 \mathrm{~s} / 31.38 \mathrm{~s}=2.97$ & 7.75 & $100 \% ; 100 \%$ \\
$\mathbf{1 1 2}$ & $112,(4,4,8)$ & $112,(4,4,8)$ & 14.49 & 12.00 & $21.81 \mathrm{~s} / 8.71 \mathrm{~s}=2.50$ & 6.60 & $92.6 \% ; 90.3 \%$ \\
$\mathbf{1 2 8}$ & $128,(1,3,5,7)$ & $128,(1,4,2,9)$ & 14.70 & 11.57 & $14.15 \mathrm{~s} / 4.43 \mathrm{~s}=3.19$ & 5.84 & $63.1 \% ; 64.8 \%$ \\
$\mathbf{1 6 0}$ & $160,(2,4,4,6)$ & $160,(3,2,3,8)$ & 13.08 & 10.33 & $7.96 \mathrm{~s} / 2.77 \mathrm{~s}=2.87$ & 4.77 & $86.8 \% ; 83.0 \%$ \\
\hline
\end{tabular}

the average observed running-time (using a SAGE implementation on a cluster of Intel Xeon X5650 2.67GHz cores) over 1000 trials for each set of parameters for experiment E. Column "T1/T2" denotes the ratio of the running-time estimate (using the estimate of Lemma 3) between two experiments. Column "succ. E1 ; E2" denotes the success probability (the probability at least one solution is found) by averaging over 1000 trials. It is noted that our improved algorithms have comparable success probability to the original CPW algorithm.

Note that the actual running-time only follows roughly from the size upper bound "\#E", but not exactly. For instance in experiment E1, dimension $m=128$ can be reduced to $\tilde{m}=112$ which gives a better size upper bound (from 14.70 to 14.49). However, the actual running-time for keeping $\tilde{m}=128$ is better than after reducing to 112. This is because the column "\#E" only denotes the theoretical estimate of the maximum bit storage. The list sizes at various levels might be smaller than "\#E" and the list sizes at the same level may be different. Note it is easy to compute the list sizes at each level using the information in Table 8 using a linear program.

\subsection{Collision on $\mathcal{B}=\{0,1\}$}

We now consider the collision problem for the set $\mathcal{B}=\{0,1\}$. This simulates the SWIFFT collision problem. Experiment E3 is the Howgrave-Graham-Joux "birthday attack" variant of the Minder-Sinclair CPW algorithm. In other words, we do the CPW algorithm using parameters $\ell_{1}, \ldots, \ell_{t}$ and then apply the birthday paradox to the final list of entries in $\mathbb{Z}_{q}^{n-\left(\ell_{1}+\cdots+\ell_{t}\right)}$. Experiment $\mathbf{E} 4$ is the same, but applying the HNF and using approximate merge. The parameters are $n=16, q=17$, and $m$ ranges from 96 to 176 . The notation used in Table 9 is analogous to that used in Table 8. Column "succ. E3 ; E4" also denotes the success probability by averaging over 1000 trials. By comparison, our improved algorithms take less time and have higher success probability than their counterparts (except for $m=128$ ).

To summarize, the experimental results presented in Tables 8 and 9 confirm that the improved algorithms using the Hermite normal form indeed perform faster in practice than the previous state-of-the-art (with comparable success probability). Our experimental results are also in agreement with the theoretical analysis. 
Table 9. Comparison of algorithms for ISIS inversion $\mathcal{B}=\{0,1\}$.

\begin{tabular}{llllllll}
\hline$m$ & $\tilde{m}$ and $\ell_{i}(\mathbf{E 3})$ & $\tilde{m}$ and $\ell_{i}(\mathbf{E 4})$ & $\# \mathbf{E 3}$ & \#E4 & T. E3 / E4 = ratio & T3/T4 & succ. E3 ; E4 \\
\hline $\mathbf{9 6}$ & $88,(2,3,4)$ & $96,(3,2,3)$ & 15.39 & 10.34 & $44.56 \mathrm{~s} / 5.09 \mathrm{~s}=8.75$ & 7.22 & $46.2 \% ; 56.0 \%$ \\
$\mathbf{1 2 8}$ & $128,(1,3,2,4)$ & $96,(3,2,3)$ & 14.95 & 10.34 & $19.79 \mathrm{~s} / 5.09 \mathrm{~s}=3.50$ & 6.29 & $95.6 \% ; 56.0 \%$ \\
$\mathbf{1 4 4}$ & $144,(1,4,3,2)$ & $144,(2,2,2,3)$ & 13.91 & 10.29 & $17.34 \mathrm{~s} / 6.73 \mathrm{~s}=2.58$ & 4.75 & $84.5 \% ; 97.7 \%$ \\
$\mathbf{1 6 0}$ & $160,(2,3,3,2)$ & $160,(3,1,2,3)$ & 12.85 & 9.94 & $8.55 \mathrm{~s} / 5.65 \mathrm{~s}=1.51$ & 1.66 & $67.5 \% ; 90.0 \%$ \\
\hline
\end{tabular}

\section{Conclusions and Further Work}

We have explained how the Hermite normal form reduces the ISIS problem to an "approximate subset-sum" problem, and we have given a variant of the CPW algorithm that can solve such problems. As a result, we have given improved algorithms for inverting and finding collisions for the SWIFFT hash function. Our new methods are approximately 500-1000 times faster than previous methods, but do not violate the security level of the SWIFFT hash function claimed in [21].

In Sect. 3 we have analyzed the HGJ and BCJ algorithms for ISIS instances of density $>1$. Figure 1 illustrates how these algorithms behave as the density grows. While these results are not of interest for the SWIFFT hash function (as it has very high density), they may be relevant to the study of other ISIS problems with small coefficient sets.

Section 4.3 discusses adapting the BCJ algorithm to the case of approximate ISIS (using the Hermite normal form of the original ISIS instance) and obtains an improved algorithm to solve ISIS. We believe these ideas will be of interest to studying ISIS instances with low density and small coefficient sets.

Finally, Sect. 7 reports on extensive experiments with the CPW algorithm. These results confirm our theoretical analysis, and demonstrate that applying the Hermite normal form to ISIS gives a significant speedup in practice.

There are several questions remaining for future work. One important challenge is to develop algorithms with lower storage requirements and that can be parallelized or distributed. We note that Pollard-rho-style random walks do not seem to be useful as they lead to running times proportional to $\sqrt{q^{n}}$, which is usually much worse than the running times considered in this paper.

It is also worthwhile to examine the lattice-based schemes submitted to the NIST Postquantum Standardization process. ${ }^{11}$ There might be schemes for which the algorithms described in this paper could be applied.

\section{Acknowledgements}

We thank the reviewers for their detailed comments and suggestions. We acknowledge NeSI (the New Zealand eScience Infrastructure), PSMN (Pôle Scientifique de Modélisation Numérique - ENS de Lyon) and the Research Computing at Florida Atlantic University for providing computing facilities and support. The work of the first author has been supported in part by ERC Starting Grant ERC-2013-StG-335086-LATTAC.

\footnotetext{
${ }^{11}$ https://csrc.nist.gov/Projects/Post-Quantum-Cryptography.
} 


\section{References}

[1] Y. Arbitman, G. Dogon, V. Lyubashevsky, D. Micciancio, C. Peikert, A. Rosen, SWIFFTX: A proposal for the SHA-3 standard. Submitted to NIST SHA-3 competition

[2] M. Ajtai, Generating hard instances of lattice problems, electronic colloquium on computational complexity (ECCC), TR96-007 (1996)

[3] N. Bansal, S. Garg, J. Nederlof, N. Vyas, Faster space-efficient algorithms for subset sum and k-sum, in Proceedings of the 49th Annual ACM SIGACT Symposium on Theory of Computing, STOC 2017 (2017), pp. 198-209

[4] A. Becker, J.-S. Coron, A. Joux, Improved generic algorithms for hard knapsacks, in K. G. Paterson (ed.), EUROCRYPT 2011. LNCS, 6632 (Springer, 2011), pp. 364-385

[5] D.J. Bernstein, Better price-performance ratios for generalized birthday attacks, in Workshop Record of SHARCS07, (2007). http://cr.yp.to/papers.html\#genbday

[6] D.J. Bernstein, T. Lange, R. Niederhagen, C. Peters, P. Schwabe, FSBday: Implementing Wagner's generalized birthday attack against the SHA-3 round-1 candidate FSB, in B.K. Roy and N. Sendrier (eds.), INDOCRYPT 2009. LNCS, 5922 (Springer, 2009), pp. 18-38

[7] J. Buchmann, R. Lindner, Secure parameters for SWIFFT, in B. Roy and N. Sendrier (eds.), INDOCRYPT 2009. LNCS, 5922 (2009), pp. 1-17

[8] P. Camion, J. Patarin, The knapsack hash function proposed at Crypto' 89 can be broken, in D.W. Davies (ed.), EUROCRYPT 1991. LNCS, 547 (Springer, 1991), pp. 39-53

[9] T.H. Cormen, C.E. Leiserson, R.L. Rivest, C. Stein, Introduction to algorithms, 2nd ed., (MIT press, 2001)

[10] M.J. Coster, A. Joux, B.A. LaMacchia, A.M. Odlyzko, C.-P. Schnorr, J. Stern, Improved low-density subset sum algorithms. Comput. Complex., 2, 111-128, (1992)

[11] I. Dinur, O. Dunkelman, N. Keller, A. Shamir, Efficient dissection of composite problems, with applications to cryptanalysis, knapsacks, and combinatorial search problems, in R. Safavi-Naini and R. Canetti, CRYPTO 2012. LNCS, 7417 (Springer, 2012), pp. 719-740.

[12] M. Finiasz, N. Sendrier, Security bounds for the design of code-based cryptosystems, In: Matsui, M. (ed.) ASIACRYPT 2009. LNCS, vol. 5912 (Springer, Heidelberg, 2009), pp. 88105

[13] N. Gama, M. Izabachène, P.Q. Nguyen, X. Xie, Structural lattice reduction: generalized worst-case to average-case reductions and homomorphic cryptosystems. EUROCRYPT (2), 528-558 (2016)

[14] N. Howgrave-Graham, J.H. Silverman, W. Whyte, A meet-in-the-middle attack on an NTRU private key, Technical Report 004, NTRU Cryptosystems, June 2003

[15] N. Howgrave-Graham, A. Joux, New generic algorithms for hard knapsacks, in H. Gilbert (ed.), EUROCRYPT 2010. LNCS, 6110 (Springer, 2010), pp. 235-256

[16] N. Howgrave-Graham, A. Joux, New Generic Algorithms for Hard Knapsacks (preprint), 17 pages (undated). Available from www.joux.biz/publications/Knapsacks.pdf

[17] N. Howgrave-Graham, A Hybrid Lattice-Reduction and Meet-in-the-Middle Attack Against NTRU, in A. Menezes (ed.), CRYPTO 2007. LNCS, 4622 (Springer, 2007), pp. 150-169.

[18] J.C. Lagarias, A.M. Odlyzko, Solving low-density subset sum problems. J. ACM 32(1), 229-246 (1985)

[19] P. Kirchner, Improved generalized birthday attack, cryptology ePrint Archive: Report 2011/377 (2011)

[20] V. Lyubashevsky, On random high density subset sums, electronic colloquium on computational complexity (ECCC) 007 (2005)

[21] V. Lyubashevsky, D. Micciancio, C. Peikert, A. Rosen, SWIFFT: A modest proposal for FFT hashing, in K. Nyberg (ed.), FSE 2008. LNCS, 5086 (Springer, 2008), pp. 54-72

[22] D. Micciancio, Generalized compact knapsacks, cyclic lattices, and efficient one-way functions from worst-case complexity assumptions, electronic colloquium on computational complexity (ECCC), No. 095 (2004)

[23] D. Micciancio, C. Peikert, Hardness of SIS and LWE with Small Parameters, in R. Canetti and J. A. Garay (eds.), CRYPTO 2013. LNCS, 8042 (Springer , 2013), pp. 21-39

[24] D. Micciancio, O. Regev, Lattice-based cryptography, in D. J. Bernstein, J. Buchmann and E. Dahmen (eds.), Post quantum cryptography, (Springer, 2009), pp. 147-191

[25] L. Minder, A. Sinclair, The extended k-tree algorithm. J. Cryptol. 25, 349-382 (2012) 
[26] R. Schroeppel, A. Shamir, A $T=O\left(2^{n / 2}\right), S=O\left(2^{n / 4}\right)$ algorithm for certain NP-complete problems. SIAM J. Comput. 3, 456-464 (1981)

[27] A. Shallue, An Improved Multi-set Algorithm for the Dense Subset Sum Problem, in A.J. van der Poorten and A. Stein (eds.), ANTS 2008. LNCS, 5011 (Springer, 2008), pp. 416-429

[28] D. Wagner, A Generalized Birthday Problem, in M. Yung (ed.), CRYPTO 2002, LNCS. 2442 (Springer, 2002), pp. 288-303

Publisher's Note Springer Nature remains neutral with regard to jurisdictional claims in published maps and institutional affiliations. 